\title{
The off-shell c-map
}

\author{
Nabamita Banerjee, ${ }^{a}$ Bernard de Wit ${ }^{b, c}$ and Stefanos Katmadas ${ }^{d, e}$ \\ ${ }^{a}$ Department of Physics, IISER Pune, \\ Homi Bhaba Road, Pashan, Pune, India \\ ${ }^{b}$ Nikhef Theory Group, \\ Science Park 105, 1098 XG Amsterdam, The Netherlands \\ ${ }^{c}$ Institute for Theoretical Physics, Utrecht University, \\ Leuvenlaan 4, 3584 CE Utrecht, The Netherlands \\ ${ }^{d}$ Dipartimento di Fisica, Università di Milano-Bicocca, \\ Piazza della Scienza 3, I-20126 Milano, Italy \\ e INFN, Sezione di Milano-Bicocca, \\ Piazza della Scienza 3, I-20126 Milano, Italy \\ E-mail: nabamita@iiserpune.ac.in, B.deWit@uu.nl, \\ stefanos.katmadas@unimib.it
}

ABSTRACT: The off-shell version of the c-map is presented, based on a systematic offshell reduction from four to three space-time dimensions for supergravity theories with eight supercharges. In the reduction, the R-symmetry group is enhanced to local $[\mathrm{SU}(2) \times$ $\mathrm{SU}(2)] / \mathbb{Z}_{2} \cong \mathrm{SO}(4)$ and the c-map is effected by a parity transformation in the internal space that interchanges the two SU(2) factors. Vector and tensor supermultiplets are each others conjugate under the c-map and both can be dualized in three dimensions to (on-shell) hypermultiplets.

As shown in this paper the off-shell formulation indeed leads to a clarification of many of the intricate issues that play a role in the c-map. The results for off-shell Lagrangians quadratic in space-time derivatives are analyzed in detail and compared to the literature. The underlying reasons are identified why not all of the four-dimensional tensor multiplet Lagrangians can be in the image of the c-map. The advantage of the off-shell approach is, that it also enables a systematic analysis of theories with higher-derivative couplings. This is demonstrated for a particular subclass of such theories, which, under certain conditions, are consistent under the c-map. In principle, explicit results for realistic four-dimensional type-II string compactifications can be explored in this way.

KEYwords: Extended Supersymmetry, Supersymmetry and Duality, String Duality

ArXiv EPRINT: 1512.06686 


\section{Contents}

1 Introduction 1

2 Off-shell dimensional reduction; the Weyl multiplet $\quad 4$

2.1 Reduction ansätze 6

2.2 Decomposition of the 4D Weyl multiplet 8

2.3 Gauge compensator and the Kaluza-Klein vector supermultiplet 12

$\begin{array}{lll}3 & N=4 \text { conformal supergravity in three dimensions } & 17\end{array}$

4 Off-shell dimensional reduction: matter multiplets 20

5 Four and three-dimensional fields and invariant Lagrangians $\quad 26$

5.1 The supercovariant dictionary: expressing $4 D$ fields in terms of $3 D$ fields $\quad 27$

$\begin{array}{ll}5.2 & \text { Lagrangians quadratic in derivatives } \\ & 30\end{array}$

5.2.1 The hypermultiplet Lagrangian 30

$\begin{array}{ll}\text { 5.2.2 The tensor multiplet Lagrangian } & 31\end{array}$

5.2.3 The vector multiplet Lagrangian $\quad 34$

$\begin{array}{lll}5.3 & \text { The c-map } & 42\end{array}$

6 The c-map for higher-derivative couplings $\quad 44$

6.1 Higher derivative couplings through composite matter multiplets 44

$\begin{array}{lll}6.2 & \text { More higher-derivative couplings } & 48\end{array}$

A Relations between 4D and 3D Riemann curvatures $\quad 50$

B The conversion of $4 D$ chiral to $\mathrm{SU}(2) \times \mathrm{SU}(2)$ covariant $3 D$ spinors $\quad 52$

\section{Introduction}

Dimensional reduction of supersymmetric theories is usually performed in the context of on-shell field representations. For theories with a large number of supercharges this is unavoidable, as off-shell representations are usually not available. For theories based on off-shell representations it is often not worthwhile to define a full and consistent off-shell dimensional reduction scheme, because the extra auxiliary fields contained in the off-shell configuration can be removed by solving their corresponding (algebraic) field equations. In the presence of higher-derivative couplings, however, these field equations are no longer algebraic. In their on-shell form these couplings will therefore take the form of an iterative expansion in ever increasing powers of space-time derivatives, which will completely obscure their underlying structure. In this case an off-shell reduction scheme is indispensable, as 
one obtains a supercovariant dictionary expressing the higher-dimensional fields into the lower-dimensional ones, so that different invariants can be reduced on a case by case basis.

The dimensional reduction of $4 D N=2$ supergravity theories to $3 D$ dimensions is special and is relevant for the so-called c-map [1]. Because the number of supersymmetries remains the same, four-dimensional theories with $N=2$ supersymmetry yield three-dimensional theories with $N=4$ supersymmetry. Dimensional reduction is usually applied to Lagrangians that are at most quadratic in space-time derivatives and the c-map has mainly been studied at the on-shell level [1-4]. In its original form it maps vector multiplets into hypermultiplets. But in its off-shell form it maps vector into tensor supermultiplets and vice versa [5]. Both these types of multiplets can be converted to hypermultiplets in $3 D$ by vector-scalar duality.

The c-map is related to T-duality for type-II string theories with one spatial dimension compactified on a circle $[6,7]$. In the compactification of type-IIA string theory the spectrum of $1 / 2$-BPS states consists of the massless states described by $9 D$ supergravity, coupled to momentum and winding states associated with the circle. Denoting the circumference of the circle by $L$, the momentum states have masses of order $1 / L$, while the winding modes have masses of order $L$. In the limit $L \rightarrow \infty$ the momentum states become massless and the theory decompactifies with massless states described by type-IIA supergravity. Obviously a second decompactification limit exists for $L \rightarrow 0$, where the winding states become massless. In the latter case the massless states are those described by typeIIB supergravity. The momentum and winding modes belong to different representations associated with different central charges of the $9 D$ supersymmetry algebra [8]. This is then consistent with the fact that the massless spectra of IIA and IIB string theory are different.

The inequivalent representations of the massless states in type-IIA and type-IIB string theory have also direct consequences for massless states when compactifying on a CalabiYau three-fold. For a Calabi-Yau manifold with Hodge numbers $h_{11}$ an $h_{12}$ the massless states of the $N=2$ four-dimensional effective field theory on the IIA side correspond to the states of $N=2$ supergravity with $h_{11}$ vector supermultiplets and $h_{12}+1$ hypermultiplets. Likewise, the massless states on the IIB side correspond to those of $N=2$ supergravity, but now with $h_{12}$ vector supermultiplets and $h_{11}+1$ hypermultiplets. Those are the two configurations that emerge in the circle decompactification limits of the type-II string theories when compactified on a Calabi-Yau space times a circle. We should mention that an additional intriguing feature of Calabi-Yau three-folds, which will not be directly relevant for this paper, is that they appear in pairs which are topologically different and related by the fact that $h_{12}$ and $h_{11}$, which define the number of complex structure moduli and of Kähler form moduli, respectively, are interchanged. This surprising phenomenon is known as mirror symmetry, and it can be combined with T-duality to obtain important results for string effective actions (for an early reference, see e.g. [9]).

Some time ago it was demonstrated how to carry out the dimensional reduction of $5 D$ off-shell supergravity field configurations with eight supercharges to the corresponding $4 D$ ones, based on a corresponding reduction of the off-shell supersymmetry algebra [10] and its representations. This reduction can be performed systematically on separate supersymmetric invariants and in particular on actions containing higher-derivative couplings. 
To accomplish the reduction one maps a supermultiplet in higher dimension to a corresponding, not necessarily irreducible, supermultiplet in lower dimension, possibly in a certain conformal supergravity background. When considering the supersymmetry algebra in the context of a lower-dimensional space-time, the dimension of the automorphism group of the algebra (the R-symmetry group) usually increases, and this has to be taken into account when casting the resulting supermultiplet in a form that is appropriate for the lower-dimensional theory.

In three dimensions, the massless matter states can be characterized in terms of vector and tensor supermultiplets (or of on-shell hypermultiplets). As is to be expected, the $4 D$ R-symmetry group $(\mathrm{SU}(2) \times \mathrm{U}(1)) / \mathbb{Z}_{2}$ is enhanced to $(\mathrm{SU}(2) \times \mathrm{SU}(2)) / \mathbb{Z}_{2} \cong \mathrm{SO}(4)$ in three dimensions. Under the c-map the two factors of the $3 D$ R-symmetry group will be interchanged and so are the vector and tensor supermultiplets. In addition the matter fields of the Weyl multiplet, two scalars and one spinor, will change sign. A similar phenomenon takes place for hypermultiplets, as their scalar fields parametrize a local product of two quaternion-Kähler spaces, each of them associated with one of the $\mathrm{SU}(2)$ factors of the Rsymmetry group. ${ }^{1}$ Some of the final results of the dimensional reduction procedure can be compared to existing results in the literature on $N=4$ (conformal) supergravity theories in three dimensions (see e.g. [15-20] where further references can be found). We will discuss the details in due course.

As in [10], the off-shell reduction scheme is subtle, especially in view of the fact that the $4 D$ Weyl multiplet decomposes into a $3 D$ Weyl multiplet and an additional (KaluzaKlein) vector multiplet. Both in four and in three dimensions, the matter multiplets are defined in a superconformal background consisting only of the $4 D$ or the $3 D$ Weyl multiplet fields, respectively. To fully establish this fact requires to also consider the transformation rules beyond the linearised approximation. The fact that the R-symmetry group is enhanced upon dimensional reduction requires a conversion of the spinor basis. Furthermore, to realize the extended R-symmetry locally it is necessary to introduce an $\mathrm{SU}(2) / \mathrm{U}(1)$ local phase factor that ensures that the $4 D$ and $3 D$ local R-symmetries can coexist. The central result of this paper is then to express the $4 D$ off-shell fields in terms of the $3 D$ ones. This leads to a covariant dictionary which enables us to write any $4 D$ supersymmetric action in terms of its $3 D$ counterparts by direct substitution. While this is relatively straightforward for hypermultiplet and tensor multiplet Lagrangians quadratic in derivatives, it is much more subtle for the vector multiplet Lagrangians. The reason is that the number of vector multiplets is increased in the reduction by the addition a Kaluza-Klein vector supermultiplet that originates from the $4 D$ Weyl multiplet. Therefore the resulting $3 D$ Lagrangian has to be completely reformulated to match the form of the generic $3 D$ tensor multiplet Lagrangians. In doing so, one establishes that the $3 D$ vector Lagrangians, although identical in structure to the $3 D$ tensor Lagrangians, belong to a restricted class. This can be inferred from the fact that they are manifestly invariant under both the vector gauge symmetry and under local R-symmetry. Moreover they are invariant under a group of rigid transformations that are characteristic for the dimensional reduction of $4 D$ vector

\footnotetext{
${ }^{1}$ Our attention will, however, not be focused on the conversion to hypermultiplets [11-14].
} 
multiplets (for an extensive classification, see [3]). None of these features are generically present in the $3 D$ tensor Lagrangians. Therefore not all the tensor multiplets can belong to the image of the c-map. The corresponding phenomenon for hypermultiplets has been noted long ago [1].

The supercovariant dictionary can straightforwardly be applied to any $4 D$ off-shell supersymmetric Lagrangian including the ones with higher-derivative couplings. We present a few examples of higher-derivative Lagrangians and discuss their implication for the cmap. In principle these results are relevant for explicit four-dimensional type-II string compactifications, such as given in [21]. This last topic definitely warrants further study, but this is outside the scope of the present paper.

This paper is organized as follows. Section 2 presents the off-shell reduction to three space-time dimensions of the $4 D$ Weyl multiplet. After a first discussion of its reduction we establish the resulting decomposition into the $3 D$ Weyl multiplet and a separate KaluzaKlein vector supermultiplet. The necessary conversion of $4 D$ into $3 D$ spinors is introduced in subsection 2.3. The resulting $3 D$ Weyl multiplet corresponds to $N=4$ conformal supergravity and is considered in detail in section 3. Its characteristic features, in particular those related to the c-map, are discussed and compared to the literature. Section 4 analyzes the reduction of the supersymmetry transformations for the various $4 D$ matter supermultiplets: the vector supermultiplet, the tensor supermultiplet and the hypermultiplet, by expressing all the $4 D$ fields into $3 D$ fields. All the results are then expressed in the form of a supercovariant dictionary, which expresses all the $4 D$ fields into the $3 D$ fields. This is done in section 5 , where we also apply the dictionary to the $4 D$ supersymmetric actions with at most two derivatives. In a third subsection we then describe the conditions upon which a $3 D$ Lagrangian can be uplifted to two inequivalent $4 D$ Lagrangians with a different field content by making use of the c-map. A more novel application concerns the reduction of higher-derivative couplings. This is the topic of section 6 where we present a few examples and discuss their properties in relation to the c-map. Finally there are two appendices. Appendix A discusses the relation between $4 D$ and $3 D$ Riemann curvatures, while the more technical aspects of $4 D$ to $3 D$ spinor conversion are presented in appendix B.

\section{Off-shell dimensional reduction; the Weyl multiplet}

Starting from the super conformal transformations for $4 D$ supermultiplets we compactify one spatial dimension on a circle which will be shrunk to zero size, so that the space-time dimension is reduced to $3 D$. Subsequently we reinterpret the results in terms of $3 D$ super conformal transformations. The first multiplet to consider is the Weyl multiplet, because it acts as a background for the other supermultiplets: the vector and tensor multiplet and the hypermultiplet. A second reason why the Weyl multiplet deserves priority, is that it becomes reducible upon the reduction, unlike the other (matter) supermultiplets. The $N=2$ Weyl multiplet in $D=4$ comprises $24+24$ bosonic and fermionic degrees of freedom, which, in the reduction to $D=3$ dimensions will decompose into the Weyl multiplet comprising $16+16$ degrees of freedom, and a vector multiplet comprising $8+8$ degrees of freedom. As we shall see, this decomposition takes a subtle form off-shell. 
The independent fields of the Weyl multiplet of four-dimensional $N=2$ conformal supergravity consist of the vierbein $e_{M}{ }^{A}$, the gravitino fields $\psi_{M}{ }^{i}$, the dilatational gauge field $b_{M}$, the R-symmetry gauge fields $\mathcal{V}_{M i}{ }^{j}$ (which is an anti-hermitian, traceless matrix in the $\mathrm{SU}(2)$ indices $i, j)$ and $A_{M}$, an anti-selfdual tensor field $T_{A B}{ }^{i j}$, a scalar field $D$ and a spinor field $\chi^{i}$. All spinor fields are Majorana spinors which have been decomposed into chiral components. Our $4 D$ conventions are as in [22]. The three gauge fields $\omega_{M}^{A B}, f_{M}{ }^{A}$ and $\phi_{M}{ }^{i}$, associated with local Lorentz transformations, conformal boosts and S-supersymmetry, respectively, are not independent as will be discussed later. The infinitesimal Q, S and K transformations of the independent fields, parametrized by spinors $\epsilon^{i}$ and $\eta^{i}$ and a vector $\Lambda_{\mathrm{K}}{ }^{A}$, respectively, are as follows, ${ }^{2}$

$$
\begin{aligned}
\delta e_{M}{ }^{A}= & \bar{\epsilon}^{i} \gamma^{A} \psi_{M i}+\bar{\epsilon}_{i} \gamma^{A} \psi_{M}{ }^{i}, \\
\delta \psi_{M}{ }^{i}= & 2 \mathcal{D}_{M} \epsilon^{i}-\frac{1}{8} T_{A B}{ }^{i j} \gamma^{A B} \gamma_{M} \epsilon_{j}-\gamma_{M} \eta^{i} \\
\delta b_{M}= & \frac{1}{2} \bar{\epsilon}^{i} \phi_{M i}-\frac{3}{4} \bar{\epsilon}^{i} \gamma_{M} \chi_{i}-\frac{1}{2} \bar{\eta}^{i} \psi_{M i}+\text { h.c. }+\Lambda_{\mathrm{K}}{ }^{A} e_{M A}, \\
\delta A_{M}= & \frac{1}{2} \mathrm{i} \bar{\epsilon}^{i} \phi_{M i}+\frac{3}{4} \mathrm{i} \bar{\epsilon}^{i} \gamma_{M} \chi_{i}+\frac{1}{2} \mathrm{i} \bar{\eta}^{i} \psi_{M i}+\text { h.c. }, \\
\delta \mathcal{V}_{M}{ }^{i}{ }_{j}= & 2 \bar{\epsilon}_{j} \phi_{M}{ }^{i}-3 \bar{\epsilon}_{j} \gamma_{M} \chi^{i}+2 \bar{\eta}_{j} \psi_{M}{ }^{i}-(\text { h.c. } ; \text { traceless }), \\
\delta T_{A B}{ }^{i j}= & 8 \bar{\epsilon}^{i i} R(Q){ }_{A B}{ }^{j]}, \\
\delta \chi^{i}= & -\frac{1}{12} \gamma^{A B} \not D T_{A B}{ }^{i j} \epsilon_{j}+\frac{1}{6} R(\mathcal{V})_{M N}{ }_{j}{ }_{j} \gamma^{M N} \epsilon^{j}-\frac{1}{3} \mathrm{i} R_{M N}(A) \gamma^{M N} \epsilon^{i} \\
& +D \epsilon^{i}+\frac{1}{12} \gamma_{A B} T^{A B i j} \eta_{j}, \\
\delta D= & \bar{\epsilon}^{i} \not D \chi_{i}+\bar{\epsilon}_{i} \not D \chi^{i} .
\end{aligned}
$$

The above supersymmetry variations and also the conventional constraints that we have to deal with in due time, depend on a number of supercovariant curvature tensors, which will be defined shortly. The full superconformally covariant derivative is denoted by $D_{M}$, while $\mathcal{D}_{M}$ denotes a covariant derivative with respect to Lorentz, dilatation, and chiral $\mathrm{SU}(2) \times \mathrm{U}(1)$ transformations, e.g.

$$
\mathcal{D}_{M} \epsilon^{i}=\left(\partial_{M}-\frac{1}{4} \omega_{M}{ }^{A B} \gamma_{A B}+\frac{1}{2} b_{M}+\frac{1}{2} \mathrm{i} A_{M}\right) \epsilon^{i}+\frac{1}{2} \mathcal{V}_{M}{ }^{i}{ }_{j} \epsilon^{j}
$$

Under local scale and U(1) transformations the various fields and transformation parameters transform as indicated in table 1 .

The gauge fields associated with local Lorentz transformations, S-supersymmetry and special conformal boosts, $\omega_{M}^{A B}, \phi_{M}{ }^{i}$ and $f_{M}{ }^{A}$, respectively, are composite and determined

\footnotetext{
${ }^{2}$ In four dimensions we consistently use world indices $M, N, \ldots$ and tangent space indices $A, B, \ldots$ For fields that do not carry such indices the distinction between $4 D$ and $3 D$ fields may not always be manifest, but it will be specified in the text whenever necessary. We use Pauli-Källén conventions with hermitian gamma matrices and label the coordinates by $x^{M}=\left(x^{4}, x^{1}, x^{2}, x^{3}\right)$, where $x^{\mu}=\left(x^{1}, x^{2}, x^{3}\right)$ with $x^{3}=\mathrm{i} x^{0}$. Consistency with the four-dimensional results that we will use requires that $\varepsilon_{4123}=1, \gamma^{1} \gamma^{2} \gamma^{3}=\gamma^{4} \gamma^{5}$ and $\varepsilon_{123}=1$. From subsection 2.3 we will employ proper $3 D$ gamma matrices, which are defined in appendix B.
} 


\begin{tabular}{|c|cccccccc|ccc|cc|}
\hline $4 D$ & \multicolumn{10}{|c|}{ Weyl multiplet } & \multicolumn{1}{|c|}{ parameters } \\
\hline field & $e_{M}{ }^{A}$ & $\psi_{M}{ }^{i}$ & $b_{M}$ & $A_{M}$ & $\mathcal{V}_{M^{i}{ }_{j}}$ & $T_{A B}{ }^{i j}$ & $\chi^{i}$ & $D$ & $\omega_{M}^{A B}$ & $f_{M}{ }^{A}$ & $\phi_{M}{ }^{i}$ & $\epsilon^{i}$ & $\eta^{i}$ \\
\hline$w$ & -1 & $-\frac{1}{2}$ & 0 & 0 & 0 & 1 & $\frac{3}{2}$ & 2 & 0 & 1 & $\frac{1}{2}$ & $-\frac{1}{2}$ & $\frac{1}{2}$ \\
\hline$c$ & 0 & $-\frac{1}{2}$ & 0 & 0 & 0 & -1 & $-\frac{1}{2}$ & 0 & 0 & 0 & $-\frac{1}{2}$ & $-\frac{1}{2}$ & $-\frac{1}{2}$ \\
\hline$\gamma_{5}$ & & + & & & & & + & & & & - & + & - \\
\hline
\end{tabular}

Table 1. Weyl and chiral weights $\left(w\right.$ and $c$ ) and fermion chirality $\left(\gamma_{5}\right)$ of the Weyl multiplet component fields and the supersymmetry transformation parameters in four space-time dimensions.

by conventional constraints. In this case these constraints are S-supersymmetry invariant and they take the following form,

$$
\begin{aligned}
R(P)_{M N}{ }^{A} & =0, \\
\gamma^{M} R(Q)_{M N}{ }^{i}+\frac{3}{2} \gamma_{N} \chi^{i} & =0, \\
e^{N}{ }_{B} R(M)_{M N A}{ }^{B}-\mathrm{i} \tilde{R}(A)_{M A}+\frac{1}{8} T_{A B i j} T_{M}{ }^{B i j}-\frac{3}{2} D e_{M A} & =0 .
\end{aligned}
$$

The curvatures appearing in (2.3) take the following form,

$$
\begin{aligned}
& R(P)_{M N}^{A}=2 \partial_{[M} e_{N]}^{A}+2 b_{[M} e_{N]}^{A}-2 \omega_{[M}^{A B} e_{N] B}-\frac{1}{2}\left(\bar{\psi}_{\left[M^{i} \gamma^{A} \psi_{N] i}+\text { h.c. }\right),}\right. \\
& R(Q)_{M N}^{i}=2 \mathcal{D}_{[M} \psi_{N]}^{i}-\gamma_{[M} \phi_{N]}^{i}-\frac{1}{8} T^{A B i j} \gamma_{A B} \gamma_{[M} \psi_{N] j},
\end{aligned}
$$

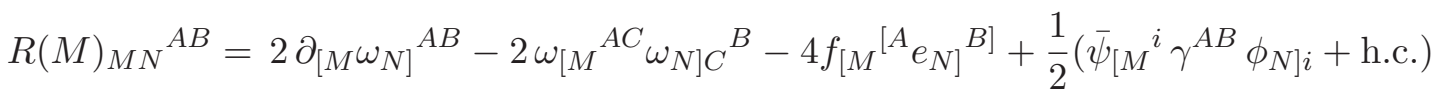

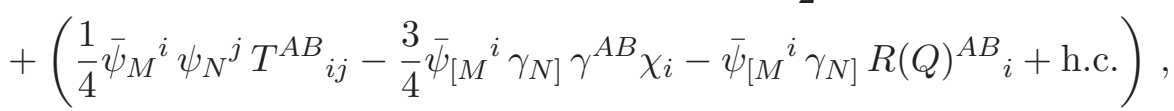

$$
\begin{aligned}
& R(A)_{M N}=2 \partial_{[M} A_{N]}-\mathrm{i}\left(\frac{1}{2} \bar{\psi}_{[M}^{i} \phi_{N] i}+\frac{3}{4} \bar{\psi}_{[M}^{i} \gamma_{N]} \chi_{i}-\text { h.c. }\right) \text {. }
\end{aligned}
$$

\subsection{Reduction ansätze}

The reduction to three space-time dimensions is effected by first carrying out the standard Kaluza-Klein decompositions on the various fields, to ensure that the resulting $3 D$ fields will transform consistently under four-dimensional diffeomorphisms. The space-time coordinates are decomposed into $x^{M} \rightarrow\left(x^{4}, x^{\mu}\right)$, where $x^{4}$ denotes the (spatial) coordinate that will be suppressed in the reduction. Subsequently the vielbein field and the dilatational gauge field are then written in special form, by means of an appropriate local Lorentz transformation and a conformal boost, respectively. In obvious notation,

$$
e_{M}^{A}=\left(\begin{array}{cc}
e_{\mu}{ }^{a} & B_{\mu} \phi^{-1} \\
0 & \phi^{-1}
\end{array}\right), \quad e_{A}^{M}=\left(\begin{array}{cc}
e_{a}^{\mu} & -e_{a}{ }^{\nu} B_{\nu} \\
0 & \phi
\end{array}\right), \quad b_{M}=\left(\begin{array}{c}
b_{\mu} \\
0
\end{array}\right) .
$$

On the right-hand side of these decompositions, we exclusively used three-dimensional notation, with world and tangent-space indices, $\mu, \nu, \ldots$ and $a, b, \ldots$, taking three values. 
Observe that the scaling weights for $e_{M}{ }^{A}$ and $e_{\mu}{ }^{a}$ are equal to $w=-1$, while for $\phi$ we have $w=1$. The fields $b_{M}$ and $b_{\mu}$ have weight $w=0$. The above formulae suffice to express the $4 D$ Riemann curvature tensor in terms of the $3 D$ Riemann tensor and the fields $\phi$ and $B_{\mu}$. The corresponding equations are collected in appendix A and will be needed later on.

We now turn to the supersymmetry transformations. Since we have imposed gauge choices on the vielbein field and the dilatational gauge field, one has to include compensating Lorentz and special conformal transformations when deriving the $3 D$ Q-supersymmetry transformations to ensure that the gauge conditions are preserved. Only the parameter of the Lorentz transformation is relevant, and it is given by,

$$
\varepsilon^{a 4}=-\varepsilon^{4 a}=-\phi\left(\bar{\epsilon}_{i} \gamma^{a} \psi^{i}+\bar{\epsilon}^{i} \gamma^{a} \psi_{i}\right),
$$

where we assumed the standard Kaluza-Klein decomposition on the gravitino fields,

$$
\psi_{M}^{i}=\left(\begin{array}{c}
\psi_{\mu}^{i}+B_{\mu} \psi^{i} \\
\psi^{i}
\end{array}\right)
$$

which ensures that $\psi_{\mu}{ }^{i}$ on the right-hand side transforms as a $3 D$ vector. Upon including the extra term (2.6), one can write down the Q- and S-supersymmetry transformations on the $3 D$ fields defined above. As a result of this, the $3 D$ and $4 D$ supersymmetry transformation will be different. For instance, the supersymmetry transformations of the $3 D$ fields $e_{\mu}^{a}, \phi$ and $B_{\mu}$ read,

$$
\begin{aligned}
\delta e_{\mu}{ }^{a} & =\bar{\epsilon}_{i} \gamma^{a} \psi_{\mu}{ }^{i}+\bar{\epsilon}^{i} \gamma^{a} \psi_{\mu i}, \\
\delta \phi & =-\phi^{2}\left(\bar{\epsilon}_{i} \gamma_{4} \psi^{i}+\bar{\epsilon}^{i} \gamma_{4} \psi_{i}\right), \\
\delta B_{\mu} & =\phi^{2}\left(\bar{\epsilon}_{i} \gamma_{\mu} \psi^{i}+\bar{\epsilon}^{i} \gamma_{\mu} \psi_{i}\right)+\phi\left(\bar{\epsilon}_{i} \gamma_{4} \psi_{\mu}{ }^{i}+\bar{\epsilon}^{i} \gamma_{4} \psi_{\mu i}\right),
\end{aligned}
$$

where the first term in $\delta B_{\mu}$ originates from the compensating transformation (2.6). Consequently the supercovariant field strength of $B_{\mu}$ contains a term that is not contained in the supercovariant four-dimensional curvature $R(P)_{M N}{ }^{A}$. Therefore the $4 D$ spin-connection components are not supercovariant with respect to $3 D$ supersymmetry, as is exhibited below,

$$
\begin{aligned}
\omega_{M}^{a b} & =\left(\begin{array}{c}
\omega_{\mu}^{a b} \\
0
\end{array}\right)+\frac{1}{2} \phi^{-2} \hat{F}(B)^{a b}\left(\begin{array}{c}
B_{\mu} \\
1
\end{array}\right) \\
\omega_{M}^{a 4} & =-\frac{1}{2}\left(\begin{array}{c}
\phi^{-1} \hat{F}(B)_{\mu}^{a}+\phi\left(\bar{\psi}_{\mu i} \gamma^{a} \psi^{i}+\bar{\psi}_{\mu}^{i} \gamma^{a} \psi_{i}\right) \\
0
\end{array}\right)-\phi^{-2} D^{a} \phi\left(\begin{array}{c}
B_{\mu} \\
1
\end{array}\right) .
\end{aligned}
$$

Here we introduced the supercovariant field strength and derivative (with respect to $3 D$ supersymmetry),

$$
\begin{aligned}
\hat{F}(B)_{\mu \nu} & =2 \partial_{[\mu} B_{\nu]}-\phi^{2}\left(\bar{\psi}_{[\mu i} \gamma_{\nu]} \psi^{i}+\bar{\psi}_{[\mu}{ }^{i} \gamma_{\nu]} \psi_{i}\right)-\phi \bar{\psi}_{[\mu}{ }^{i} \gamma_{4} \psi_{\nu] i} \\
D_{\mu} \phi & =\left(\partial_{\mu}-b_{\mu}\right) \phi+\frac{1}{2} \phi^{2}\left(\bar{\psi}_{\mu i} \gamma_{4} \psi^{i}-\bar{\psi}_{\mu}{ }^{i} \gamma_{4} \psi_{i}\right) .
\end{aligned}
$$

Subsequently we write down corresponding Kaluza-Klein decompositions for some of the other fields of the Weyl multiplet, which do not require special gauge choices,

$$
\mathcal{V}_{M^{i}{ }_{j}}=\left(\begin{array}{c}
\mathcal{V}_{\mu}{ }^{i}{ }_{j}+B_{\mu} \mathcal{V}^{i}{ }_{j} \\
\mathcal{V}^{i}{ }_{j}
\end{array}\right), \quad A_{M}=\left(\begin{array}{c}
A_{\mu}+B_{\mu} A \\
A
\end{array}\right), \quad \phi_{M}{ }^{i}=\left(\begin{array}{c}
\phi_{\mu}{ }^{i}+B_{\mu} \phi^{i} \\
\phi^{i}
\end{array}\right) \text {. }
$$


Furthermore we define two complex $3 D$ target-space vectors $A_{a}^{ \pm}$such that

$$
\begin{array}{ll}
T_{a 4}{ }^{i j}=A_{a}^{-} \varepsilon^{i j}, & T_{a b}{ }^{i j}=\varepsilon_{a b c} A^{-c} \varepsilon^{i j}, \\
T_{a 4 i j}=A_{a}^{+} \varepsilon_{i j}, & T_{a b i j}=-\varepsilon_{a b c} A^{+c} \varepsilon_{i j},
\end{array}
$$

where $A_{a}^{+}$and $A_{a}^{-}$are related by complex conjugation. Here one has to bear in mind that we are using Pauli-Källén notation, so that $A_{a}^{+}+A_{a}^{-}$is real when $a$ denotes a spatial component, and imaginary when $a$ denotes the time component. This is reflected in the different sign in the last two terms of (2.12). All gamma matrices are hermitian. We recall that the convention for the Levi-Civita tensors is $\varepsilon^{4 a b c}=\varepsilon^{a b c}$, and $\varepsilon^{123}=1$. Correspondingly for the gamma matrices we have the conventions that $\gamma_{5}=\gamma_{4} \gamma_{1} \gamma_{2} \gamma_{3}$ and we note the useful relation $\gamma^{a b}=\varepsilon^{a b c} \gamma_{c} \gamma_{4} \gamma_{5}$, so that e.g. $T_{A B}{ }^{i j} \gamma^{A B}=2 A_{a}^{-} \gamma^{a} \gamma_{4}\left(1+\gamma_{5}\right) \varepsilon^{i j}$.

\subsection{Decomposition of the 4D Weyl multiplet}

Hence we are now ready to consider the Q- and S-supersymmetry transformations of the spinor fields originating from the $4 D$ gravitino fields. Up to possible higher-order spinor terms, one derives from $(2.1)$,

$$
\begin{aligned}
\delta \psi^{i}= & {\left[-\frac{1}{2} \phi^{-2} \hat{F}(B)_{a b} \gamma^{a b}+\phi^{-2} \not D \phi \gamma_{4}+\mathrm{i} A\right] \epsilon^{i}+\mathcal{V}^{i}{ }_{j} \epsilon^{j}-\phi^{-1} A^{-} \varepsilon^{i j} \epsilon_{j} } \\
& -\phi^{-1} \gamma_{4}\left(\eta^{i}+\frac{1}{2} A^{-} \gamma_{4} \varepsilon^{i j} \epsilon_{j}-\frac{1}{4} \phi^{-1} \hat{F}(B)_{a b} \gamma^{a b} \gamma_{4} \epsilon^{i}\right), \\
\delta \psi_{\mu}{ }^{i}= & 2\left(\partial_{\mu}-\frac{1}{4} \omega_{\mu}{ }^{a b} \gamma_{a b}+\frac{1}{2} b_{\mu}+\frac{1}{2} \mathrm{i} A_{\mu}-\frac{1}{8} \mathrm{i} \phi^{-1} \hat{F}(B)_{\mu}\right) \epsilon^{i}+\mathcal{V}_{\mu}{ }_{j}{ }_{j} \epsilon^{j}+A_{\mu}^{-} \varepsilon^{i j} \gamma_{4} \epsilon_{j} \\
& -\gamma_{\mu}\left(\eta^{i}+\frac{1}{2} A^{-} \gamma_{4} \varepsilon^{i j} \epsilon_{j}-\frac{1}{4} \phi^{-1} \hat{F}(B)_{a b} \gamma^{a b} \gamma_{4} \epsilon^{i}\right),
\end{aligned}
$$

where $\hat{F}(B)_{\mu}=\mathrm{i} e \varepsilon_{\mu \nu \rho} \hat{F}(B)^{\nu \rho}$. Although the results (2.8) and (2.13) are still incomplete, they already exhibit some of the systematic features that will turn out to be universal. Therefore let us first have a brief perusal of these initial results.

The fields whose transformations we have determined will belong to two $3 D$ supermultiplets, namely the Weyl and the Kaluza-Klein vector multiplet. Clearly, the fields $e_{\mu}{ }^{a}$ and $\psi_{\mu}{ }^{i}$ belong to the Weyl multiplet, whereas $\phi, B_{\mu}$ and $\psi^{i}$ belong to the vector multiplet. An obvious puzzle is the fact that we have identified only one real scalar, whereas the $3 D$ vector multiplet contains three scalars. This is related to a generic feature of dimensional reduction, namely that lower-dimensional results are often obtained in a gauge-fixed version of the (local) R-symmetry group. Another aspect of this phenomenon is that the vector fields $A^{ \pm}{ }_{a}$ seem to play the role of a complex gauge field, because they appear to covariantize the derivatives on $\phi$ and $\epsilon^{i}$ in (2.13), in spite of the fact that the $A^{ \pm}{ }_{a}$ are actually auxiliary fields in $D=4$. As we shall see shortly, $A^{ \pm}{ }_{a}$ combined with $A_{\mu}$ will provide the $\mathrm{SU}(2)$ gauge fields associated with the enhancement of the $\mathrm{U}(1)$ factor of the $4 D$ R-symmetry group. This additional $\mathrm{SU}(2)$ group emerges in the reduction, in addition to the manifest $\mathrm{SU}(2) \mathrm{R}$-symmetry group of the $4 D$ theory. Hence the full $3 D$ R-symmetry group equals $(\mathrm{SU}(2) \times \mathrm{SU}(2)) / \mathbb{Z}_{2} \cong \mathrm{SO}(4)$. This situation is in close analogy to what was 
encountered in five dimensions [10], where the $\mathrm{SU}(2)$ R-symmetry group was enhanced to $\mathrm{SU}(2) \times \mathrm{U}(1) / \mathbb{Z}_{2}$. Observe that in both cases the fermions remain irreducible under the extended R-symmetry group.

Just as in [10], we will discover that the higher-dimensional supersymmetry transformations yield the lower-dimensional ones, but with parameters that involve additional field-dependent terms. These field-dependent terms can be dropped eventually. We see this already in the uniform field-dependent additions to the S-supersymmetry transformations in (2.13) and we will discover similar modifications of the R-symmetry transformations in due course. Some of those can be interpreted as compensating transformations related to the fact that the formulation that we obtain is gauge-fixed. This gauge-fixing will be removed at the end by introducing a local $\mathrm{SU}(2) / \mathrm{U}(1)$ phase factor, which provides the missing two fields to the Kaluza-Klein vector multiplet.

To demonstrate some of this in more detail, let us present the higher-order completion of (2.13). The pattern that we find is repeated in the results for the matter supermultiplets that will be presented in section 4. Explicit calculation leads to the following results,

$$
\begin{aligned}
\delta\left(\varepsilon_{i j} \phi^{2} \psi^{j}\right)= & -\frac{1}{2} \hat{F}(B)_{a b} \gamma^{a b} \varepsilon_{i j} \epsilon^{j}+\gamma^{\mu}\left(D_{\mu} \phi \gamma_{4} \varepsilon_{i j} \epsilon^{j}+\mathcal{A}_{\mu}^{-} \phi \epsilon_{i}\right)+\mathrm{i} C \phi \varepsilon_{i j} \epsilon^{j}+Y_{i}{ }^{j 0} \varepsilon_{j k} \epsilon^{k} \\
& -\phi \gamma_{4} \varepsilon_{i j}\left(\eta^{j}+\tilde{\eta}^{j}\right)+\frac{1}{2} \tilde{\Lambda}_{i}{ }^{j}\left(\varepsilon_{j k} \phi^{2} \psi^{k}\right)+\frac{1}{2} \tilde{\Sigma}^{-} \varepsilon_{i j} \gamma_{4}\left(\varepsilon^{j k} \phi^{2} \psi_{k}\right) \\
\delta \psi_{\mu}{ }^{i}= & 2\left(\partial_{\mu}-\frac{1}{4} \omega_{\mu}{ }^{a b} \gamma_{a b}+\frac{1}{2} b_{\mu}+\frac{1}{2} \mathrm{i} \mathcal{A}_{\mu}{ }^{0}\right) \epsilon^{i}+\mathcal{V}_{\mu}{ }^{i}{ }_{j} \epsilon^{j}+\mathcal{A}_{\mu}^{-} \varepsilon^{i j} \gamma_{4} \epsilon_{j} \\
& -\gamma_{\mu}\left(\eta^{i}+\tilde{\eta}^{i}\right)+\frac{1}{2} \tilde{\Lambda}^{i}{ }_{j} \psi_{\mu}{ }^{j}-\frac{1}{2} \tilde{\Sigma}^{-} \varepsilon^{i j} \gamma_{4} \psi_{\mu j} .
\end{aligned}
$$

Here we have used the definition,

$$
\begin{aligned}
C & =\phi A-\frac{1}{2} \mathrm{i} \phi^{2} \bar{\psi}^{k} \gamma_{4} \psi_{k}, \\
Y_{j}^{i}{ }^{0} & =\phi^{2}\left(\mathcal{V}^{i}{ }_{j}+3 \phi \bar{\psi}^{i} \gamma_{4} \psi_{j}-\frac{3}{2} \phi \bar{\psi}^{l} \gamma_{4} \psi_{l} \delta^{i}{ }_{j}\right) .
\end{aligned}
$$

Obviously the field $Y^{0 i}{ }_{j}$ will correspond to the auxiliary field of the Kaluza-Klein vector multiplet. The field $C$ will turn out to belong to the $3 D$ Weyl multiplet.

Furthermore we have introduced six vector fields which are related to the two sets of $\mathrm{SU}(2)$ gauge fields associated with the $3 D$ R-symmetry group,

$$
\begin{aligned}
\mathcal{A}_{\mu}{ }^{0}= & A_{\mu}-\frac{1}{4} \phi^{-1} \hat{F}(B)_{\mu}-\frac{1}{2} \mathrm{i} \phi^{2} \bar{\psi}^{k} \gamma_{\mu} \psi_{k}, \\
\mathcal{A}_{\mu}{ }^{-}= & e_{\mu}{ }^{a} A_{a}^{-}+\phi \varepsilon_{i j} \bar{\psi}_{\mu}{ }^{i} \psi^{j}+\frac{1}{2} \phi^{2} \varepsilon_{i j} \bar{\psi}^{i} \gamma_{\mu} \gamma_{4} \psi^{j}, \\
\mathcal{A}_{\mu}{ }^{+}= & e_{\mu}{ }^{a} A_{a}^{+}+\phi \varepsilon^{i j} \bar{\psi}_{\mu i} \psi_{j}+\frac{1}{2} \phi^{2} \varepsilon^{i j} \bar{\psi}_{i} \gamma_{\mu} \gamma_{4} \psi_{j}, \\
\mathcal{V}_{\mu}{ }^{i}{ }_{j}= & \left.\mathcal{V}_{\mu}{ }^{i}{ }_{j}\right|_{4 D}+\phi\left(\bar{\psi}_{\mu}{ }^{i} \gamma_{4} \psi_{j}+\bar{\psi}^{i} \gamma^{4} \psi_{\mu j}-\frac{1}{2} \delta^{i}{ }_{j}\left(\bar{\psi}_{\mu}{ }^{k} \gamma_{4} \psi_{k}+\bar{\psi}^{k} \gamma^{4} \psi_{\mu k}\right)\right) \\
& +\phi^{2}\left(\bar{\psi}^{i} \gamma_{\mu} \psi_{j}-\frac{1}{2} \delta^{i}{ }_{j} \bar{\psi}^{k} \gamma_{\mu} \psi_{k}\right) .
\end{aligned}
$$


The remaining quantities are given by

$$
\begin{aligned}
\tilde{\eta}^{i}= & \frac{1}{2} A^{-} \gamma_{4} \varepsilon^{i j} \epsilon_{j}-\frac{1}{4} \phi^{-1} \hat{F}(B)_{a b} \gamma^{a b} \gamma_{4} \epsilon^{i} \\
& +\frac{1}{2} \phi^{2}\left(\bar{\psi}^{(i} \psi^{j)} \epsilon_{j}+\bar{\psi}^{i} \gamma^{a} \psi_{j} \gamma_{a} \epsilon^{j}+\bar{\psi}^{i} \gamma_{4} \psi_{j} \gamma_{4} \epsilon^{j}\right), \\
\tilde{\Lambda}_{i}^{j}= & 2 \phi\left(\bar{\epsilon}_{i} \gamma_{4} \psi^{j}+\bar{\psi}_{i} \gamma_{4} \epsilon^{j}-\frac{1}{2} \delta_{i}{ }^{j}\left(\bar{\epsilon}_{k} \gamma_{4} \psi^{k}+\bar{\psi}_{k} \gamma_{4} \epsilon^{k}\right)\right), \\
\tilde{\Sigma}^{-}= & \left(\tilde{\Sigma}^{+}\right)^{*}=2 \phi \varepsilon_{i j} \bar{\epsilon}^{i} \psi^{j},
\end{aligned}
$$

and are related to the various field-dependent transformations mentioned above. They will appear universally for all fields and define the decomposition of the $4 D$ Q-supersymmetry variations, in terms of the $3 D$ Q-supersymmetry variations combined with a field-dependent S-supersymmetry transformation, a field-dependent SU(2) R-symmetry transformation, and a field-dependent $\mathrm{SU}(2) / \mathrm{U}(1)$ chiral transformation. The latter should be regarded as compensating transformations associated with the fact that the reduction leads to a gauge-fixed formulation with respect to the new (local) R-symmetry transformations,

$$
\left.\delta_{\mathrm{Q}}(\epsilon)\right|_{4 D} ^{\text {reduced }} \Psi=\left.\delta_{\mathrm{Q}}(\epsilon)\right|_{3 D} \Psi+\left.\delta_{\mathrm{S}}(\tilde{\eta})\right|_{3 D} \Psi+\left.\delta_{\mathrm{SU}(2)}(\tilde{\Lambda})\right|_{3 D} \Psi+\left.\delta_{\mathrm{SU}(2) / \mathrm{U}(1)}(\tilde{\Sigma})\right|_{3 D} \Psi .
$$

To give a meaning to the right-hand side one has to identify fields $\Psi$ that transform covariantly in the $3 D$ setting, so that all transformations in the above decomposition are clearly defined. The identification of these fields is done iteratively. Here one has to realize that the $4 D$ transformations for the Weyl multiplet are defined in a background consisting of the $4 D$ Weyl multiplet, whereas the $3 D$ transformations of the matter multiplets are defined in the $3 D$ background. But the field-dependent parameters in (2.18) still depend on a variety of the $4 D$ Weyl multiplet fields. When these parameters are associated with proper $3 D$ symmetries they can be safely suppressed and this is what we will do henceforth. Obviously this concerns the parameters $\tilde{\eta}^{i}$ and $\tilde{\Lambda}_{i}{ }^{j}$, but not $\tilde{\Sigma}^{ \pm}$. The fate of $\tilde{\Sigma}^{ \pm}$will be become clear shortly in the next subsection 2.3.

Let us examine some further properties of the newly defined fields (2.15) and (2.16) before proceeding. First of all, an explicit calculation reveals the following transformations under S-supersymmetry,

$$
\begin{aligned}
\delta_{\mathrm{S}} C & =0 \\
\delta_{\mathrm{S}} Y^{i}{ }_{j}{ }^{0} & =\phi^{2}\left(\bar{\psi}^{i} \eta_{j}-\psi_{j} \eta^{i}\right)-\frac{1}{2} \delta^{i}{ }_{j} \phi^{2}\left(\bar{\psi}^{k} \eta_{k}-\bar{\psi}_{k} \eta^{k}\right), \\
\delta_{\mathrm{S}} \mathcal{A}_{\mu}{ }^{0} & =\frac{1}{2} \mathrm{i}\left(\bar{\psi}_{\mu i} \eta^{i}-\bar{\psi}_{\mu}{ }^{i} \eta_{i}\right), \\
\delta_{\mathrm{S}} \mathcal{A}_{\mu}{ }^{-} & =-\varepsilon_{i j} \bar{\psi}_{\mu}{ }^{i} \gamma_{4} \eta^{j}, \\
\delta_{\mathrm{S}} \mathcal{A}_{\mu}{ }^{+} & =-\varepsilon^{i j} \bar{\psi}_{\mu i} \gamma_{4} \eta_{j}, \\
\delta_{\mathrm{S}} \mathcal{V}_{\mu}{ }^{i}{ }_{j} & =\bar{\psi}_{\mu}{ }^{i} \eta_{j}-\bar{\psi}_{\mu j} \eta^{i}-\frac{1}{2} \delta^{i}{ }_{j}\left(\bar{\psi}_{\mu}{ }^{k} \eta_{k}-\bar{\psi}_{\mu k} \eta^{k}\right) .
\end{aligned}
$$

Note that the S-supersymmetry transformations of the fields $\mathcal{A}_{\mu}{ }^{0}, \mathcal{A}_{\mu}^{ \pm}$and $\mathcal{V}_{\mu}{ }^{i}{ }_{j}$ are very similar, which confirms that they will indeed provide the connections associated with the 
$\mathrm{SU}(2) \times \mathrm{SU}(2)$ R-symmetry group. Note also that the Q- and S-supersymmetry transformations of the gravitini in (2.14) no longer contain any auxiliary fields, but only the connections associated with the local Lorentz group, dilatations, and R-symmetry.

The structure of the $3 D$ Weyl supermultiplet is almost covered completely at this stage, except for the auxiliary spinor $\chi^{i}$ and the scalar $D$. To see what they will represent in the $3 D$ theory, let us consider the variation of the S-invariant scalar $C$, defined in (2.15). Under Q-supersymmetry it transforms as

$$
\delta C=\frac{1}{2} \mathrm{i} \bar{\epsilon}^{i} \gamma_{4} \breve{\chi}_{i}+\text { h.c. }
$$

where $\breve{\chi}_{i}$ equals

$$
\begin{aligned}
\breve{\chi}_{i}= & \left.\frac{5}{2} \gamma_{4} \chi_{i}\right|_{4 D} \\
& +\frac{2}{3} \phi \gamma_{4} \not D \psi_{i}+\frac{2}{3} \phi A^{+} \varepsilon_{i j} \psi^{j}+\mathrm{i} \phi^{2} A \psi_{i}-\not D \phi \gamma_{4} \psi_{i}+\phi^{2} \mathcal{V}_{i}^{j} \psi_{j}-\frac{1}{12} \hat{F}(B)_{a b} \gamma^{a b} \psi_{i}
\end{aligned}
$$

where the right-hand side is expressed in terms of the original $4 D$ fields and covariant derivatives. In this result we used that the components of the $4 D$ S-supersymmetry gauge field are given by (up to terms cubic in fermions)

$$
\begin{aligned}
\phi_{\hat{4}}{ }^{i}= & \left.\phi^{-1} \gamma_{4} \chi^{i}\right|_{4 D}+\frac{2}{3} \not D \psi^{i}+\frac{1}{6} \phi^{-1} \gamma^{a b} F(B)_{a b} \gamma_{4} \psi^{i}-\frac{1}{6} A^{-} \gamma_{4} \varepsilon^{i j} \psi_{j}, \\
\phi_{\mu}{ }^{i}= & \left.\phi_{\mu}{ }^{i}\right|_{3 D}+\frac{1}{2} A^{-} \gamma_{4} \varepsilon^{i j} \psi_{\mu j}-\frac{1}{4} \phi^{-1} \hat{F}(B)_{a b} \gamma^{a b} \gamma_{4} \psi_{\mu}{ }^{i}+\frac{1}{2} \hat{F}(B)_{a b} \gamma^{a b} \gamma_{\mu} \psi^{i}+\left.\frac{1}{2} \gamma_{\mu} \chi^{i}\right|_{4 D} \\
& -\frac{2}{3} \phi \gamma_{4}\left(e_{\mu}^{a}+\gamma^{a} \gamma_{\mu}\right)\left(D_{a} \psi^{i}+\frac{1}{4} \phi^{-1} \hat{F}(B)_{a b} \gamma^{b} \gamma_{4} \psi^{i}+\frac{1}{4} \gamma_{4} A^{-} \gamma_{a} \varepsilon^{i j} \psi_{j}\right) .
\end{aligned}
$$

The definition of the $3 D$ S-supersymmetry gauge field $\phi_{\mu}{ }^{i}$ will be discussed in section 3 . The scalar field of the Weyl multiplet related to the $4 D$ scalar $D$ can be identified by analyzing the supersymmetry transformations on the spinor $\breve{\chi}_{i}$ defined in $(2.21)$,

$$
\begin{aligned}
\delta \breve{\chi}^{i}= & 2 \mathrm{i} \gamma_{4} \not D C \epsilon^{i}+\left.D\right|_{3 D} \epsilon^{i}+\frac{1}{2} \gamma_{4} \gamma_{c} \varepsilon^{a b c} R(\mathcal{V})_{a b}{ }^{i} \epsilon^{j} \\
& -\frac{1}{2} \gamma_{4} \gamma_{c} \varepsilon^{a b c} e_{a}{ }^{\mu} e_{b}{ }^{\nu}\left[\left(2 \partial_{\mu} \mathcal{A}_{\nu}{ }^{-}+\mathrm{i} \mathcal{A}_{\mu}{ }^{0} \mathcal{A}_{\nu}{ }^{-}\right) \gamma_{4} \varepsilon^{i j} \epsilon_{j}+\mathrm{i}\left(R_{\mu \nu}\left(\mathcal{A}^{0}\right)+\mathrm{i} \mathcal{A}_{\mu}{ }^{-} \mathcal{A}_{\nu}{ }^{+}\right) \epsilon^{i}\right] \\
& +2 \mathrm{i} C \gamma_{4} \eta^{i}-\frac{1}{2} \tilde{\Sigma}^{-} \varepsilon^{i j} \gamma_{4} \breve{\chi}_{j},
\end{aligned}
$$

where the scalar $\left.D\right|_{3 D}$ equals

$$
D=\left.2 D\right|_{4 D}-\frac{2}{3} \phi D_{a} D^{a} \phi^{-1}+\phi^{2}\left(D_{a} \phi^{-1}\right)^{2}+\frac{1}{6} \phi^{-2} F(B)_{a b}{ }^{2}+C^{2}+\frac{1}{2} \phi^{-2} Y^{0 i}{ }_{j} Y^{0 j}{ }_{i} .
$$

Neither in (2.23) nor in (2.24) did we include higher-order fermionic terms. Observe that the bracket in the second line of (2.23) will lead to the field strengths associated with the new $\mathrm{SU}(2)_{\mathrm{R}}$ symmetry. Here and in the formulae below we will include the $3 D$ conformal gauge field $f_{\mu}{ }^{a}$ in the second-order covariant derivatives, $D_{\mu} D^{a} \phi=\mathcal{D}_{\mu} \mathcal{D}^{a} \phi+w f_{\mu}{ }^{a} \phi$, where $w=1$ is the Weyl weight of $\phi$. The gauge field $f_{\mu}{ }^{a}$ will be defined explicitly in section 3 . 
We now give the expressions for the components of the $4 D$ conformal gauge fields $f_{M}{ }^{A}$. The first one, $f_{\mu}{ }^{a}$ is defined in terms of the $3 D$ fields by

$$
\begin{aligned}
f_{\mu}{ }^{a}= & \left.f_{\mu}{ }^{a}\right|_{3 D}+\frac{1}{4} \phi^{-2}\left[F(B)^{a c} F(B)_{\mu c}-\frac{1}{12} e_{\mu}^{a} F(B)_{b c}{ }^{2}\right]+\frac{1}{2} \phi\left[D_{\mu} D^{a} \phi^{-1}-\frac{1}{3} e_{\mu}{ }^{a} D_{b} D^{b} \phi^{-1}\right] \\
& -\frac{1}{4} \mathrm{i} \varepsilon_{\mu}{ }^{a b} D_{b}\left(C \phi^{-1}\right)+\frac{1}{8}\left[A^{+}{ }_{\mu} A^{-a}+A^{-}{ }_{\mu} A^{+a}-e_{\mu}{ }^{a} A^{+}{ }_{b} A^{-b}\right]-\left.\frac{1}{4} e_{\mu}{ }^{a} D\right|_{4 D},
\end{aligned}
$$

up to fermionic terms. The remaining components are $f_{\hat{4}}^{a}, f_{\mu}^{4}$ and $f_{\hat{4}}^{4}$, and are given by (we recall that 4 denotes the tangent space index and $\hat{4}$ the world index associated with the compactified coordinate)

$$
\begin{aligned}
f_{\hat{4}}{ }^{a}= & -\frac{1}{4} \phi^{-1} D_{b}(\omega) F(B)^{a b}+\frac{3}{4} \phi^{-2} F(B)^{a b} D_{b} \phi-\frac{1}{4} \mathrm{i} \varepsilon^{a b c}\left[R(A)_{b c}+C \phi^{-1} F(B)_{b c}\right] \\
& +\frac{1}{8} \varepsilon^{a b c} A^{-}{ }_{b} A^{+}{ }_{c} \\
f_{\mu}{ }^{4}= & -\frac{1}{4} \phi^{-1} D^{\nu}(\omega) F(B)_{\mu \nu}+\frac{3}{4} \phi^{-2} F(B)_{\mu \nu} D^{\nu} \phi+\frac{1}{4} \mathrm{i} \varepsilon_{\mu a b}\left[R(A)^{a b}+C \phi^{-1} F(B)^{a b}\right] \\
& +\frac{1}{8} \varepsilon_{\mu a b} A^{-a} A^{+b} \\
f_{\hat{4}}{ }^{4}= & \frac{1}{3} D_{a} D^{a} \phi^{-1}-\frac{7}{48} \phi^{-3} F(B)_{a b}{ }^{2}-\left.\frac{1}{4} \phi^{-1} D\right|_{4 D}+\frac{1}{8} \phi^{-1} A_{a}^{-} A^{+a} .
\end{aligned}
$$

With the exception of the last equation in (2.26), all the linear combinations of $D_{a} D_{b} \phi$ and $\left(D_{a} \phi\right)^{2}$ appearing in equations $(2.24),(2.25)$ and (2.26) are conformally invariant.

\subsection{Gauge compensator and the Kaluza-Klein vector supermultiplet}

At several occasions it was already pointed out that the $4 D$ R-symmetry group is enhanced to a larger symmetry group upon dimensional reduction. More specifically the $\mathrm{U}(1)$ factor of the $4 D$ R-symmetry group is extended to the group $\mathrm{SU}(2)$. Hence in $3 D$ one is dealing with two $\mathrm{SU}(2)$ factors in the R-symmetry group, one that was originally present in $4 D$ and another one that emerges in the reduction. Therefore $3 D$ spinors will carry two indices, namely one index denoted by $i, j, \ldots=1,2$ that is carried already by the $4 D$ fields, and an additional index denoted by $p, q, \ldots=+,-$, that indicates the $4 D \mathrm{U}(1)$ charge to be equal to $+\frac{1}{2}$ or $-\frac{1}{2}$, respectively. Every $3 D$ spinor can thus be written as $\Psi^{i p}$. It satisfies a Majorana constraint, so that it comprises eight components, just as in the $N=2,4 D$ setting. Here it is crucial that spinors in $3 D$ Minkowski space are real two-component spinors. The expressions for the $3 D$ spinors in terms of $4 D$ ones involve an arbitrary phase factor and the relative phase factors between the spinors belonging to different $3 D$ supermultiplets will eventually follow from insisting on uniformity of the Rsymmetry assignments in various supersymmetry transformations. The group-theoretical aspects of all this is described in detail in appendix B, where we also present the relation between the $4 D$ and $3 D$ gamma matrices.

However, at this stage the new R-symmetry transformations are not realized locally, whereas the ones originating from $4 D$ are, as is standard in the superconformal formulation. This phenomenon is well known and was, for instance, also observed in the dimensional 
reduction from five to four dimensions [10]. As it turns out the resulting lower-dimensional theory is always obtained in a gauge where all the new gauge degrees of freedom are put to zero. In the case at hand, this can be avoided by simply re-introducing the missing gauge degrees of freedom. This is done by introducing a new field $\Phi^{p}{ }_{q}$, which is an element of $\mathrm{SU}(2)$,

$$
\Phi \in \mathrm{SU}(2) \Longrightarrow \Phi \Phi^{\dagger}=\mathbb{1}_{2}, \quad \operatorname{det} \Phi=1 .
$$

and which is assigned the following transformation under the new local $\mathrm{SU}(2)$ and the original local U(1),

$$
\Phi \rightarrow V \Phi\left(\begin{array}{cc}
\mathrm{e}^{-\mathrm{i} \Lambda_{\mathrm{A}} / 2} & 0 \\
0 & \mathrm{e}^{\mathrm{i} \Lambda_{\mathrm{A}} / 2}
\end{array}\right),
$$

were $V$ denotes the new (local) $\mathrm{SU}(2)_{\mathrm{R}}$ transformation and $\Lambda_{\mathrm{A}}$ denotes the parameter of the original $\mathrm{U}(1)_{\mathrm{R}}$ group. Obviously, when fixing $\Phi$ to the identity, there is only one gauge transformation that is left unaffected, corresponding to the diagonal U(1) subgroup. Subsequently we require that $\Phi$ transforms as follows under Q-supersymmetry,

$$
\Phi^{-1} \delta \Phi=\frac{1}{2}\left(\begin{array}{cc}
0 & -\tilde{\Sigma}^{+} \\
\tilde{\Sigma}^{-} & 0
\end{array}\right)
$$

where $\tilde{\Sigma}^{ \pm}$is defined in (2.17). It is important to observe that, when proceeding to the special gauge $\Phi=\mathbb{1}$, one will induce compensating $\mathrm{SU}(2)$ transformations proportional to $\tilde{\Sigma}^{ \pm}$in the supersymmetry transformation. This implies that in the fully local version of the extended R-symmetry, those terms will cancel. In due course we see that this is indeed the case. We will subsequently redefine all spinors by multiplying them with $\Phi$, so that they will transform locally under $\mathrm{SU}(2) \times \mathrm{SU}(2)$. Before doing so we have to specify the correct $3 D$ spinor basis for the various fields. For the fields corresponding to the $4 D$ fields $\psi_{\mu}{ }^{i}$ and $\psi^{i}$, the spinor parameters of Q- and S-supersymmetry $\epsilon^{i}$ and $\eta^{i}$, their conjugate spinors, the S-supersymmetry spinor gauge fields $\phi_{\mu}{ }^{i}$ and $\phi_{\mu i}$, and the matter spinors of the Weyl multiplet, denoted by $\breve{\chi}^{i}$, the required expressions follow from appendix B,

$$
\begin{aligned}
\psi_{\mu}^{i p} & =\left(\begin{array}{c}
\varepsilon^{i j} \gamma_{4} \psi_{\mu j} \\
\psi_{\mu}{ }^{i}
\end{array}\right), & & \psi^{i p}=\phi^{2}\left(\begin{array}{c}
\varepsilon^{i j} \gamma_{4} \psi_{j} \\
\psi^{i}
\end{array}\right), \\
\epsilon^{i p} & =\left(\begin{array}{c}
\varepsilon^{i j} \gamma_{4} \epsilon_{j} \\
\epsilon^{i}
\end{array}\right), & \eta^{i p} & =\left(\begin{array}{c}
-\mathrm{i} \varepsilon^{i j} \eta_{j} \\
\mathrm{i} \gamma_{4} \eta^{i}
\end{array}\right), \\
\bar{\epsilon}_{i p} & =\left(\begin{array}{c}
\mathrm{i} \varepsilon_{i j} \bar{\epsilon}^{j} \\
-\mathrm{i} \bar{\epsilon}_{i} \gamma_{4}
\end{array}\right), & \bar{\eta}_{i p} & =\left(\begin{array}{c}
\varepsilon_{i j} \bar{\eta}^{j} \\
\bar{\eta}_{i} \gamma_{4}
\end{array}\right) . \\
\phi_{\mu}{ }^{i p} & =\left(\begin{array}{c}
-\mathrm{i} \varepsilon^{i j} \phi_{\mu j} \\
\mathrm{i} \gamma_{4} \phi_{\mu}{ }^{i}
\end{array}\right), & \chi^{i p} & =\left(\begin{array}{c}
\varepsilon^{i j} \gamma_{4} \breve{\chi}_{j} \\
\breve{\chi}^{i}
\end{array}\right) .
\end{aligned}
$$

Redefining the spinors will also affect the bosonic expressions that emerge upon applying supersymmetry and there will be extra terms in the supersymmetry transformations 
proportional to $\tilde{\Sigma}^{ \pm}$as a result of $(2.29)$, which cancel corresponding terms in the Qsupersymmetry transformation rules (2.14) and (2.23) originating from the $4 D$ transformations. This cancellation is a non-trivial check on the correctness of our strategy. A first example of the modification of the bosonic fields concerns the gauge fields $\mathcal{A}_{\mu}{ }^{p}$ associated with the new $\mathrm{SU}(2)_{\mathrm{R}}$, which follow from the second equation in (2.14), and take the form,

$$
\Phi^{-1}\left(\mathbb{1} \partial_{\mu}+\frac{1}{2} \mathcal{A}_{\mu}\right) \Phi=\frac{1}{2}\left(\begin{array}{cc}
-\mathrm{i} \mathcal{A}_{\mu}{ }^{0} & -\mathcal{A}_{\mu}{ }^{+} \\
\mathcal{A}_{\mu}{ }^{-} & \mathrm{i} \mathcal{A}_{\mu}{ }^{0}
\end{array}\right)
$$

where the quantities on the right-hand side are the ones obtained previously from the $4 D$ theory, which were listed in (2.16). From the above result one can directly derive an equation for the field strengths associated with the new $\mathrm{SU}(2)_{\mathrm{R}}$ gauge fields,

$$
\begin{aligned}
\Phi^{-1} & \left(\partial_{[\mu} \mathcal{A}_{\nu]}+\frac{1}{2} \mathcal{A}_{[\mu}, \mathcal{A}_{\nu]}\right) \Phi \\
& =\left(\begin{array}{cc}
-\mathrm{i} \partial_{[\mu} \mathcal{A}_{\nu]}^{0} & -\partial_{\mu} \mathcal{A}_{\nu]}{ }^{+} \\
\partial_{[\mu} \mathcal{A}_{\nu]}^{-} & \mathrm{i} \partial_{[\mu} \mathcal{A}_{\nu]}{ }^{0}
\end{array}\right)+\frac{1}{2}\left(\begin{array}{cc}
-\mathrm{i} \mathcal{A}_{[\mu}{ }^{0} & -\mathcal{A}_{[\mu}{ }^{+} \\
\mathcal{A}_{[\mu}{ }^{-} & \mathrm{i} \mathcal{A}_{[\mu}{ }^{0}
\end{array}\right)\left(\begin{array}{cc}
-\mathrm{i} \mathcal{A}_{\nu]}{ }^{0} & -\mathcal{A}_{\nu]}{ }^{+} \\
\mathcal{A}_{\nu]} & \mathrm{i} \mathcal{A}_{\nu]}{ }^{0}
\end{array}\right)
\end{aligned}
$$

Obviously the gauge fields $\mathcal{A}_{\mu}$ transform under local SU(2) transformations as

$$
\mathcal{A}_{\mu} \rightarrow V \mathcal{A}_{\mu} V^{-1}-2 \partial_{\mu} V V^{-1} .
$$

Likewise, the scalar field $\phi$ originating from the $4 D$ metric will now be extended to a triplet of scalar fields encoded in an anti-hermitian matrix $L_{q}^{p}$ that transforms under the new SU(2) R-symmetry. Subsequently we use the phase factor $\Phi$ to define $L^{0 p}{ }_{q}$,

$$
L_{q}^{p}{ }^{0}=\Phi\left(\begin{array}{cc}
-\mathrm{i} \phi & 0 \\
0 & \mathrm{i} \phi
\end{array}\right) \Phi^{-1}, \quad L^{0} \rightarrow\left(L^{0}\right)^{\prime}=V L^{0} V^{-1},
$$

which now transforms consistently under $\mathrm{SU}(2)$ and is invariant under the $4 D \mathrm{U}(1) \mathrm{R}$ symmetry. Let us first decompose the triplet $L^{p}{ }_{q}^{0}$ according to $[5,11]$

$$
L_{q}^{p}{ }^{0}(x, v, \bar{v})=\left(\begin{array}{cc}
-\frac{1}{2} \mathrm{i} x^{0} & v^{0} \\
-\bar{v}^{0} & \frac{1}{2} \mathrm{i} x^{0}
\end{array}\right),
$$

A priori there is no restriction on the sign of $x^{0}$ as the phase factor $\Phi$ can also change $x^{0}$ to $-x^{0}$. Under the new $\mathrm{SU}(2) \mathrm{R}$-symmetry $L^{p}{ }_{q}^{0}$ transforms as specified in (2.34). For infinitesimal transformations defined as

$$
V \approx \mathbb{1}+\frac{1}{2}\left(\begin{array}{cc}
\mathrm{i} \Sigma^{0} & \Sigma^{+} \\
-\Sigma^{-} & -\mathrm{i} \Sigma^{0}
\end{array}\right)
$$

where $\Sigma^{0}$ is real and $\Sigma^{-}=\left(\Sigma^{+}\right)^{*}$, the components $x^{0}, v^{0}$ and $\bar{v}^{0}$ thus transform as a triplet,

$$
\delta_{\mathrm{SU}(2)} v^{0}=\mathrm{i}\left(\Sigma^{0} v^{0}+\frac{1}{2} \Sigma^{+} x^{0}\right), \quad \delta_{\mathrm{SU}(2)} x^{0}=\mathrm{i}\left(\Sigma^{-} v^{0}-\Sigma^{+} \bar{v}^{0}\right) .
$$


The SU(2) covariant derivative then equals

$$
\mathcal{D}_{\mu} L^{0}=\partial_{\mu} L^{0}+\frac{1}{2}\left[\mathcal{A}_{\mu}, L^{0}\right] .
$$

Let us now return to (2.8) and consider the supersymmetry transformation of $L_{q}^{p}{ }_{q}^{0}$. We first note that $\delta \phi$ in terms of the spinors (2.30) takes the following form,

$$
\delta \phi=\mathrm{i} \bar{\epsilon}_{i+} \psi^{i+}-\mathrm{i} \bar{\epsilon}_{i-} \psi^{i-} .
$$

The supersymmetry transformation of $L^{p} q^{0}$ then follows from combining (2.29) with (2.39),

$$
\delta L^{p}{ }_{q}^{0}=2 \bar{\epsilon}_{i q} \psi^{i p}-\delta^{p}{ }_{q} \bar{\epsilon}_{i r} \psi^{i r}
$$

where the spinors have been modified by including the phase factor $\Phi$ by $\psi^{i p} \rightarrow \Phi^{p}{ }_{q} \psi^{i q}$, so that they will transform consistently under all the local R-symmetry transformations. Hence the Kaluza-Klein vector multiplet consists of the three fields contained in $L^{p}{ }_{q}^{0}$, together with the modified spinor, $\psi^{i p}$, the gauge field $B_{\mu}$ and the triplet of auxiliary fields $Y_{j}^{i}{ }_{j}^{0}$ defined in (2.15). We can now continue and consider the variation of the spinor $\psi^{i p}$, the vector field $B_{\mu}$, and the auxiliary fields $Y_{j}^{i}{ }_{j}^{0}$ using the same conventions. In that way one finds,

$$
\begin{aligned}
\delta \psi^{i p} & =\not D L^{p}{ }_{q}{ }^{0} \epsilon^{i q}-\frac{1}{2} \hat{F}(B)_{a b} \gamma^{a b} \epsilon^{i p}+C L_{q}^{p}{ }^{0} \epsilon^{i q}+Y^{i}{ }_{j}^{0} \epsilon^{j p}+L_{q}^{p}{ }^{0} \eta^{i q}, \\
\delta B_{\mu} & =\bar{\epsilon}_{i p} \gamma_{\mu} \psi^{i p}+L^{p}{ }_{q}^{0} \bar{\epsilon}_{i p} \psi_{\mu}{ }^{i q}, \\
\delta Y^{i}{ }_{j}{ }^{0} & =2 \bar{\epsilon}_{j p} \not D \psi^{i p}-L^{p}{ }^{0}{ }^{0} \bar{\epsilon}_{j p} \chi^{i q}-2 C \bar{\epsilon}_{j p} \psi^{i p}-\bar{\eta}_{j p} \psi^{i p}-(\text { trace }),
\end{aligned}
$$

where the $3 D$ gamma matrices are defined in appendix B. Here we employ a supercovariant and $\mathrm{SU}(2)$ covariant derivative, defined by

$$
D_{\mu} L_{q}^{p}{ }^{0}=\left(\partial_{\mu}-b_{\mu}\right) L^{p}{ }_{q}^{0}+\frac{1}{2} \mathcal{A}_{\mu}{ }^{p}{ }_{r} L_{q}{ }^{0}-\frac{1}{2} \mathcal{A}_{\mu}{ }^{r}{ }_{q} L^{p}{ }_{r}^{0}-\left(\bar{\psi}_{\mu i q} \psi^{i p}-\text { trace }\right) .
$$

Note that, because of the fermion redefinitions that involve the phase factor $\Phi$, the terms in the supersymmetry transformation rules proportional to $\tilde{\Sigma}^{ \pm}$have disappeared. Furthermore, none of the fields transform under the U(1) local symmetry of the $4 D$ theory. This completes the derivation of the $3 D$ Kaluza-Klein vector supermultiplet.

Apart from ensuring that the $4 D$ and $3 D$ fields can transform consistently under their respective R-symmetry groups, the role of the phase factor $\Phi$ is also to sweep out the vector defined by (2.34) over a sphere $S^{2}$ such that it will take the form (2.35). This requirement fixes the phase factor in terms of the tensor $L^{p}{ }_{q}^{0}$ up to a single phase which is related to the $\mathrm{U}(1)_{\mathrm{R}}$ local symmetry of the $4 D$ theory. The result is as follows,

$$
\begin{gathered}
\Phi_{q}^{p}\left(x^{0}, v^{0}, \bar{v}^{0}\right)=\frac{1}{\sqrt{2 L^{0}\left(L^{0}+\frac{1}{2} x^{0}\right)}}\left(\begin{array}{cc}
\mathrm{e}^{-\mathrm{i} \Lambda_{\mathrm{A}} / 2}\left(L^{0}+\frac{1}{2} x^{0}\right) & -\mathrm{e}^{\mathrm{i} \Lambda_{\mathrm{A}} / 2} \mathrm{i} v^{0} \\
-\mathrm{e}^{-\mathrm{i} \Lambda_{\mathrm{A}} / 2} \mathrm{i} \bar{v}^{0} & \mathrm{e}^{\mathrm{i} \Lambda_{\mathrm{A}} / 2}\left(L^{0}+\frac{1}{2} x^{0}\right)
\end{array}\right), \\
\phi=L^{0} \equiv \sqrt{\operatorname{det}\left[L^{0} p_{q}\right]}=\sqrt{-\frac{1}{2} L^{0 p_{q} L^{0 q}}=\sqrt{\left|v^{0}\right|^{2}+\frac{1}{4}\left(x^{0}\right)^{2}}}
\end{gathered}
$$


Note that there is no obvious singularity in the limit $v^{0} \rightarrow 0$ when $x^{0}>0$. In that case $\Phi$ becomes equal to the identity matrix. Considering the same limit when $x^{0}<0$, there are obviously some factors that become singular in (2.43), but the final result for the phase factor remains well defined and tends to a different finite matrix, whose effect is to interchange the two two eigenvalues of the matrix $L^{p} q^{0}$ in (2.35).

Since the phase factor $\Phi$ is only defined up to the phase $\Lambda_{\mathrm{A}}$, which is related to the exact $\mathrm{U}(1)_{\mathrm{R}}$ symmetry of the $4 D$ theory, it is an element of the $\mathrm{SU}(2) / \mathrm{U}(1)$ coset space, which is indeed isomorphic to the sphere $S^{2}$. This aspect gives rise to some subtle features. For instance, we have already derived that the $\mathrm{SU}(2)$ acts on $\left(x^{0}, v^{0}, \bar{v}^{0}\right)$ according to (2.37), while on the other hand we have defined the $\mathrm{SU}(2)$ transformation on $\Phi$ in (2.28). However, it turns out that the change of $\Phi$ induced by the transformations of $\left(x^{0}, v^{0}, \bar{v}^{0}\right)$ will only be consistent with (2.28), if one introduces at the same time a change of the phase $\Lambda_{\mathrm{A}}$. To see this we explicitly perform the transformations (2.37) on $\Phi$ and note that they are subject to the following equation,

$$
\delta_{\mathrm{SU}(2)} \Phi=\frac{1}{2}\left(\begin{array}{cc}
\mathrm{i} \Sigma^{0} & \Sigma^{+} \\
-\Sigma^{-} & -\mathrm{i} \Sigma^{0}
\end{array}\right) \Phi+\delta \Lambda_{\mathrm{A}} \frac{\partial \Phi}{\partial \Lambda_{\mathrm{A}}},
$$

where

$$
\delta \Lambda_{\mathrm{A}}=\Sigma^{0}-\frac{\Sigma^{+} \bar{v}^{0}+\Sigma^{-} v^{0}}{2\left(L^{0}+\frac{1}{2} x^{0}\right)} .
$$

The first term on the right-hand side of (2.44) corresponds to (2.28).

The same structure is repeated for all scalar triplets, since the phase factor $\Phi$ is used to consistently translate the $4 \mathrm{D}$ fields to $3 \mathrm{D}$ fields that are covariant with respect to the emergent $\mathrm{SU}(2)$. In order to illustrate this, let us now consider the following convenient formula for a general triplet, $(x, v, \bar{v})$, that is repeatedly used later on,

$$
\begin{aligned}
& \Phi^{-1}\left(x^{0}, v^{0}, \bar{v}^{0}\right) L(x, v, \bar{v}) \Phi\left(x^{0}, v^{0}, \bar{v}^{0}\right) \\
& \quad=\frac{1}{2 L^{0}}\left(\begin{array}{cc}
-\frac{1}{2} \mathrm{i}\left(x x^{0}+2 v \bar{v}^{0}+2 \bar{v} v^{0}\right) & -x v^{0}+v x^{0}-\frac{\bar{v} v^{0}-v \bar{v}^{0}}{L^{0}+\frac{1}{2} x^{0}} v^{0} \\
x \bar{v}^{0}-\bar{v} x^{0}-\frac{\bar{v} v^{0}-v \bar{v}^{0}}{L^{0}+\frac{1}{2} x^{0}} \bar{v}^{0} & \frac{1}{2} \mathrm{i}\left(x x^{0}+2 v \bar{v}^{0}+2 \bar{v} v^{0}\right)
\end{array}\right) .
\end{aligned}
$$

Here we have suppressed the phase factor parametrized by $\Lambda_{\mathrm{A}}$, which is subject to the exact R-symmetry of the $4 D$ theory. The result (2.46) indeed reduces to (2.35) when $x=x^{0}$ and $v=v^{0}$, upon identifying $\phi$ with $L^{0}$, thus confirming the correctness of (2.43). Under the $\mathrm{SU}(2)$ transformations (2.37) the expression in (2.46) is, however, not invariant. As follows from (2.44) the phase $\Lambda_{\mathrm{A}}$ is again switched on under the $\mathrm{SU}(2)$ transformations (2.37) by an amount $\delta \Lambda_{\mathrm{A}}$ specified by (2.45), thus leading to

$$
\begin{aligned}
& \delta_{\mathrm{SU}(2)}\left(\Phi^{-1}\left(x^{0}, v^{0}, \bar{v}^{0}\right) L(x, v, \bar{v}) \Phi\left(x^{0}, v^{0}, \bar{v}^{0}\right)\right) \\
& \quad=\frac{1}{2} \mathrm{i} \delta \Lambda_{\mathrm{A}}\left[\left(\begin{array}{cc}
1 & 0 \\
0 & -1
\end{array}\right),\left(\Phi^{-1}\left(x^{0}, v^{0}, \bar{v}^{0}\right) L(x, v, \bar{v}) \Phi\left(x^{0}, v^{0}, \bar{v}^{0}\right)\right)\right] .
\end{aligned}
$$


This is the expected result, because it indicates that the $3 D \mathrm{SU}(2)$ transformation of a $4 D$ field takes the result of a field-dependent $\mathrm{U}(1)$ transformation associated with the $4 D$ R-symmetry.

Substituting (2.43) into (2.31) one obtains the explicit expressions for the $4 D$ quantities $\mathcal{A}_{\mu}{ }^{0}$ and $\mathcal{A}_{\mu}{ }^{-}$, defined in (2.16). In principle this result must be expressed in terms of the $\mathrm{SU}\left(2\right.$ covariant derivatives of the components of $L^{p}{ }_{q}\left(x^{0}, v^{0}, \bar{v}^{0}\right)$, but in view of the above, this will only be the case up to a field-dependent $\mathrm{U}(1)$ transformation of $\mathcal{A}_{\mu}{ }^{0}$ and $\mathcal{A}_{\mu}{ }^{-}$. To exhibit the complexities let us first calculate the explicit expressions for $\mathcal{A}_{\mu}{ }^{0}$ and $\mathcal{A}_{\mu}{ }^{-}$, following the definition (2.31),

$$
\begin{aligned}
& \mathcal{A}_{\mu}^{-}=\frac{-\mathrm{i} \bar{v}^{0}\left(v^{0} \overleftrightarrow{\mathcal{D}}_{\mu} \bar{v}^{0}\right)+\mathrm{i}\left(L^{0}+\frac{1}{2} x^{0}\right)\left(\bar{v}^{0} \stackrel{\leftrightarrow}{\mathcal{D}}_{\mu} x^{0}\right)}{2\left(L^{0}\right)^{2}\left(L^{0}+\frac{1}{2} x^{0}\right)}, \\
& \mathcal{A}_{\mu}{ }^{0}=\frac{1}{2 L^{0}\left(L^{0}+\frac{1}{2} x^{0}\right)}\left\{\mathrm{i} v^{0} \overleftrightarrow{\mathcal{D}}_{\mu} \bar{v}^{0}+\operatorname{Tr}\left[\mathcal{A}_{\mu}\left(\begin{array}{cc}
\mathrm{i}\left(L^{0}+\frac{1}{2} x^{0}\right) & -v^{0} \\
\bar{v}^{0} & -\mathrm{i}\left(L^{0}+\frac{1}{2} x^{0}\right)
\end{array}\right)\right]\right\},
\end{aligned}
$$

where covariant derivatives are defined according to (2.38) and we again suppressed the phase $\Lambda_{\mathrm{A}}$. As is indicated by the structure of (2.47) the $4 D$ quantity $\mathcal{A}_{\mu}{ }^{-}$transforms covariantly under $\mathrm{SU}(2)$. However, this is not the case for $\mathcal{A}_{\mu}{ }^{0}$ in view of the fact that the definition (2.31) contains a space-time derivative which has not been made explicit in the master formula (2.46). Indeed explicit calculation reveals that $\mathcal{A}_{\mu}{ }^{-}$and $\mathcal{A}_{\mu}{ }^{0}$ transform as follows under $\mathrm{SU}(2)$,

$$
\delta_{\mathrm{SU}(2)} \mathcal{A}_{\mu}{ }^{0}=\partial_{\mu} \delta \Lambda_{\mathrm{A}}, \quad \delta_{\mathrm{SU}(2)} \mathcal{A}_{\mu}^{ \pm}= \pm \mathrm{i} \delta \Lambda_{\mathrm{A}} \mathcal{A}_{\mu}^{ \pm},
$$

which takes precisely the form of the $4 D$ infinitesimal $\mathrm{U}(1)$ transformation. We should emphasize that the matrix-valued $\mathrm{SU}(2)$ connection $\mathcal{A}_{\mu}{ }^{p}$ is implicitly contained in the covariant derivatives on the right-hand side of (2.48) and only appears explicitly in the last term for $\mathcal{A}_{\mu}{ }^{0}$.

\section{$3 \quad N=4$ conformal supergravity in three dimensions}

In the previous section we have already identified all the fields belonging the the $3 D$ Weyl multiplet. For the composite gauge fields associated with S-supersymmetry and conformal boosts, $\phi_{\mu}{ }^{i p}$ and $f_{\mu}{ }^{a}$, we did not yet present explicit expressions. The proper $3 D$ spinor field $\phi_{\mu}{ }^{i p}$ follows from the same redefinition that led to $\eta^{i p}$ given in (2.30), followed by a multiplication with the matrix $\Phi$. The additional fermion field $\chi$ was defined in (2.21), and its $3 D$ definition was already given in (2.30) (again, up to the uniform multiplication with the matrix $\Phi)$.

Rather than to recast the $4 D$ fields into the $3 D$ fields we present the resulting $3 D$ superconformal theory directly in $3 D$. We emphasize that the results we are about to describe are consistent with results previously reported in the literature, such as in [1518]. In particular they fully reproduce the non-linear results that were obtained in $[19,20]$. 


\begin{tabular}{|c|cccccccc|ccc|cc|}
\hline $3 D$ & \multicolumn{10}{|c|}{ Weyl multiplet } & \multicolumn{2}{|c|}{ parameters } \\
\hline field & $e_{\mu}{ }^{a}$ & $\psi_{\mu}{ }^{i p}$ & $b_{\mu}$ & $\mathcal{V}_{\mu j}{ }^{i} j$ & $\mathcal{A}_{\mu}{ }^{p} q$ & $C$ & $\chi^{i p}$ & $D$ & $\omega_{M}^{A B}$ & $f_{M}{ }^{A}$ & $\phi_{M}{ }^{i}$ & $\epsilon^{i}$ & $\eta^{i}$ \\
\hline$w$ & -1 & $-\frac{1}{2}$ & 0 & 0 & 0 & 1 & $\frac{3}{2}$ & 2 & 0 & 1 & $\frac{1}{2}$ & $-\frac{1}{2}$ & $\frac{1}{2}$ \\
\hline
\end{tabular}

Table 2. Weyl multiplet component fields and supersymmetry parameters with their corresponding Weyl weights in three space-time dimensions.

We start with the Q- and S-supersymmetry transformations and the conformal boosts, acting on the independent fields,

$$
\begin{aligned}
\delta e_{\mu}{ }^{a} & =\bar{\epsilon}_{i p} \gamma^{a} \psi_{\mu}{ }^{i p} \\
\delta \psi_{\mu}{ }^{i p} & =2 \mathcal{D}_{\mu} \epsilon^{i p}-\gamma_{\mu} \eta^{i p} \\
\delta b_{\mu} & =\frac{1}{2} \bar{\epsilon}_{i p} \phi_{\mu}{ }^{i p}-\frac{1}{2} \bar{\eta}_{i p} \psi_{\mu}{ }^{i p}+\Lambda_{\mathrm{K}}{ }^{a} e_{\mu a}, \\
\delta \mathcal{V}_{\mu}{ }^{i}{ }_{j} & =\bar{\epsilon}_{j p} \phi_{\mu}{ }^{i p}-2 C \bar{\epsilon}_{j p} \psi_{\mu}{ }^{i p}-\bar{\epsilon}_{j p} \gamma_{\mu} \chi^{i p}+\bar{\eta}_{j p} \psi_{\mu}{ }^{i p}-(\text { trace }), \\
\delta \mathcal{A}_{\mu}{ }^{p}{ }^{i p} & =\bar{\epsilon}_{i q} \phi_{\mu}{ }^{i p}+2 C \bar{\epsilon}_{i q} \psi_{\mu}{ }^{i p}+\bar{\epsilon}_{i q} \gamma_{\mu} \chi^{i p}+\bar{\eta}_{i q} \psi_{\mu}{ }^{i p}-(\text { trace }), \\
\delta C & =\frac{1}{2} \bar{\epsilon}_{i p} \chi^{i p} \\
\delta \chi^{i p} & =2 \not D C \epsilon^{i p}+D \epsilon^{i p}+\frac{1}{2} R(\mathcal{A})_{a b}{ }^{p}{ }^{i p} \gamma^{a b} \epsilon^{i q}-\frac{1}{2} R(\mathcal{V})_{a b}{ }_{j}{ }_{j} \gamma^{a b} \epsilon^{j p}+2 C \eta^{i p}, \\
\delta D & =\bar{\epsilon}_{i p} \not D \chi^{i p}-\bar{\eta}_{i p} \chi^{i p},
\end{aligned}
$$

where $\mathcal{V}_{\mu}{ }^{i}$ and $\mathcal{A}_{\mu}{ }^{p} q$ are the $\mathrm{SU}(2) \times \mathrm{SU}(2)$ R-symmetry gauge fields with corresponding field strengths $R(\mathcal{V})_{\mu \nu}{ }^{i}{ }_{j}$ and $R(\mathcal{A})_{\mu \nu}{ }^{p}{ }_{q}$, respectively. Furthermore we will use covariant derivatives with respect to Lorentz, dilatation, and R-symmetry transformations, such as

$$
\mathcal{D}_{\mu} \epsilon^{i p}=\left(\partial_{\mu}-\frac{1}{4} \omega_{\mu}{ }^{a b} \gamma_{a b}+\frac{1}{2} b_{\mu}\right) \epsilon^{i p}+\frac{1}{2} \mathcal{V}_{\mu}{ }_{j}{ }_{j} \epsilon^{j p}+\frac{1}{2} \mathcal{A}_{\mu}{ }^{p}{ }_{q} \epsilon^{i q},
$$

while $D_{\mu}$ denotes the covariant derivative with respect to all superconformal symmetries. We stress that the Q-supersymmetry transformation of $b_{\mu}$ does not coincide with the result that one obtains from the $4 D$ variation given in (2.1). This is not an issue because the difference can be viewed as a field-dependent shift that can be absorbed into the conformal boosts. Since $b_{\mu}$ is the only independent field that transforms under conformal boosts, this has no effect somewhere else, other than that it changes the field-dependent terms in the commutation relations (which we are not making use of explicitly). Finally the scalar fields $C$ and $D$ were identified in the $4 D$ theory in (2.15) and (2.24), respectively. We have summarized the field content of the $3 D$ Weyl multiplet in table 2 .

Note that the two $\mathrm{SU}(2)_{\mathrm{R}}$ gauge fields do not appear symmetrically in (3.1), as the terms proportional to the auxiliary fields are odd under the exchange of indices. We therefore find that the Weyl multiplet is symmetric under the interchange

$$
\mathcal{V}_{\mu}{ }^{i}{ }_{j} \longleftrightarrow \mathcal{A}_{\mu}{ }^{i}{ }_{j}, \quad C \rightarrow-C, \quad \chi^{i p} \rightarrow-\chi^{i p}, \quad D \rightarrow-D,
$$

while the vielbein and the gravitini are invariant. Similar properties have been observed before in three-dimensional extended supergravity [16], but actually this property could 
also have been inferred from [15] (see also [17, 18]). Altogether this Weyl multiplet has $16+16$ degrees of freedom. The gravitational field $e_{\mu}{ }^{a}$, the R-symmetry connections and the two scalar fields comprise 2, 6, 6, 2 bosonic degrees of freedom, respectively. The gravitini, and the spinor $\chi$ comprise 4 and 4 fermionic degrees of freedom, respectively. The covariant fields are described by a real scalar superfield of Weyl weight $w=1$, subject to the constraint that the second-order superspace derivative $D_{\alpha}{ }^{i p} D^{\alpha j q}$ is proportional to the trace with respect to the $\mathrm{SO}(4)$ indices $[18,20]$. Furthermore the transformation (3.3) can be understood as a parity transformation in the internal euclidean four-space parametrized by coordinates $X^{I} \sim X^{i p}$. The fields $C$ and $D$ transform both as pseudoscalars under this parity operation whereas the R-symmetry gauge fields consist of linear combinations of three vectors and three pseudovectors. Note that the $3 D$ results that have appeared in the literature are usually in the context of the $\mathrm{SO}(N)$ R-symmetry group; the case $N=4$ is special because the R-symmetry group factorizes.

As in $4 D$, the gauge fields associated with local Lorentz transformations, S-supersymmetry and special conformal boosts, $\omega_{\mu}^{a b}, \phi_{\mu}{ }^{i p}$ and $f_{\mu}{ }^{a}$, respectively, are composite and determined by conventional constraints. These constraints are S-supersymmetry invariant and they take the following form,

$$
R(P)_{\mu \nu}^{a}=R(Q)_{\mu \nu}^{i p}=R(M)_{\mu \nu}^{a b}=0,
$$

where the relevant curvatures appearing in (3.4) are given by

$$
\begin{aligned}
& R(P)_{\mu \nu}^{a}=2 \partial_{[\mu} e_{\nu]}^{a}+2 b_{[\mu} e_{\nu]}^{a}-2 \omega_{[\mu}^{a b} e_{\nu] b}-\frac{1}{2} \bar{\psi}_{[\mu i p} \gamma^{a} \psi_{\nu]}^{i p}, \\
& R(Q)_{\mu \nu}^{i p}=2 \mathcal{D}_{[\mu} \psi_{\nu]}^{i p}-\gamma_{[\mu} \phi_{\nu]}^{i p}, \\
& R(M)_{\mu \nu}^{a b}=2 \partial_{[\mu} \omega_{\nu]}^{a b}-2 \omega_{[\mu}^{a c} \omega_{\nu] c}^{b}-4 f_{[\mu}^{[a} e_{\nu]}^{b]}+\frac{1}{2} \bar{\psi}_{[\mu i p} \gamma^{a b} \phi_{\nu]}^{i p} .
\end{aligned}
$$

The constraints (3.4) can be solved directly,

$$
\begin{aligned}
\omega_{\mu}^{a b} & =-2 e^{\nu[a} \partial_{[\mu} e_{\nu]}^{b]}-e^{\nu[a} e^{b] \sigma} e_{\mu c} \partial_{\sigma} e_{\nu}^{c}-2 e_{\mu}{ }^{[a} e^{b] \nu} b_{\nu}-\frac{1}{4}\left(2 \bar{\psi}_{\mu i p} \gamma^{[a} \psi^{b] i p}+\bar{\psi}_{i p}^{a} \gamma_{\mu} \psi^{b i p}\right), \\
\phi_{\mu}{ }^{i p} & =\frac{1}{2} \gamma^{\rho \sigma} \gamma_{\mu} \mathcal{D}_{\rho} \psi_{\sigma}{ }^{i p} \\
f_{\mu}{ }^{a} & =R(\omega, e)_{\mu}{ }^{a}-\frac{1}{4} e_{\mu}{ }^{a} R(\omega, e)+\frac{1}{2} e_{b}^{\nu} \bar{\psi}_{[\mu i p} \gamma^{a b} \phi_{\nu]}{ }^{i p}-\frac{1}{8} e_{\mu}{ }^{a} \bar{\psi}_{\rho i p} \gamma^{\rho \sigma} \phi_{\sigma}{ }^{i p}
\end{aligned}
$$

where $R(\omega, e)_{\mu}^{a}=R(\omega)_{\mu \nu}^{a b} e_{b}^{\nu}$ is the non-symmetric Ricci tensor, and $R(\omega, e)$ the corresponding Ricci scalar. The curvature $R(\omega)_{\mu \nu}^{a b}$ is associated with the spin connection field $\omega_{\mu}^{a b}$.

The transformations of $\omega_{\mu}^{a b}, \phi_{\mu}^{i p}$ and $f_{\mu}^{a}$ are induced by the constraints (3.4). We present their Q- and S-supersymmetry variations up to terms cubic in fermions, as well as 
the transformations under conformal boosts, as

$$
\begin{aligned}
\delta \omega_{\mu}{ }^{a b}= & -\frac{1}{2} \bar{\epsilon}_{i p} \gamma^{a b} \phi_{\mu}{ }^{i p}-\frac{1}{2} \bar{\eta}_{i p} \gamma^{a b} \psi_{\mu}{ }^{i p}+2 \Lambda_{\mathrm{K}}{ }^{[a} e_{\mu}{ }^{b]}, \\
\delta \phi_{\mu}{ }^{i p}= & -2 f_{\mu}{ }^{a} \gamma_{a} \epsilon^{i p}+\frac{1}{2} \gamma^{a b} \gamma_{\mu}\left(R(\mathcal{V})_{a b}{ }^{i}{ }_{j} \epsilon^{j p}+R(\mathcal{A})_{a b}{ }^{p}{ }_{q} \epsilon^{i q}\right) \\
& +2 \mathcal{D}_{\mu} \eta^{i p}+\Lambda_{\mathrm{K}}{ }^{a} \gamma_{a} \psi_{\mu}{ }^{i p}, \\
\delta f_{\mu}{ }^{a}= & -\frac{1}{2} \bar{\epsilon}_{i p} \gamma^{a b} R(S)_{\mu b}{ }^{i p}+\frac{1}{4} \mathrm{i} \varepsilon^{a b c} \bar{\epsilon}_{i p}\left(R(\mathcal{V})_{b c}{ }^{i}{ }_{j} \psi_{\mu}{ }^{j p}+R(\mathcal{A})_{b c}{ }^{p}{ }_{q} \psi_{\mu}{ }^{i q}\right) \\
& +\frac{1}{2} \bar{\eta}_{i p} \gamma^{a} \phi_{\mu}{ }^{i p}+\mathcal{D}_{\mu} \Lambda_{\mathrm{K}}{ }^{a} .
\end{aligned}
$$

Note that the curvature, $R(S)_{\mu b}{ }^{i p}$, of the S-supersymmetry gauge field appears explicitly, as one cannot solve for it in terms of other fields. This is familiar from the curvature $R(M)_{\mu \nu}{ }^{a b}$ in higher dimensional theories, but in the three dimensional theory this property extends to the field strengths of all composite gauge fields.

In order to exhibit this difference with higher dimensions, we consider the Bianchi identities for $R(P)_{\mu \nu}{ }^{a}, R(Q)_{\mu \nu}{ }^{i p}$ and $R(M)_{\mu \nu}{ }^{a b}$, which lead to

$$
\begin{aligned}
& D_{[a} R(P)_{b c]}^{d}+R(M)_{[b c a]}^{d}=\delta_{[a}^{d} R(D)_{b c]} \Rightarrow R(D)_{a b}=0, \\
& D_{[a} R(Q)_{b c]}^{i p}=\gamma_{[a} R(S)_{b c]}^{i p} \Rightarrow \gamma^{a b} R(S)_{a b}^{i p}=0, \\
& \varepsilon^{a b c} D_{a} R(M)_{b c}{ }^{d e}=2 \varepsilon^{b c[d} R(K)_{b c}^{e]} \Rightarrow \varepsilon^{b c[d} R(K)_{b c}^{e]}=0 \text {, }
\end{aligned}
$$

where in the second step in each line we used the conventional constraints (3.4), to obtain simple constraints on the curvatures $R(D)_{\mu \nu}, R(S)_{\mu \nu}{ }^{i p}$ and $R(K)_{\mu \nu}{ }^{a}$. In particular, the constraints for $R(S)_{\mu \nu}{ }^{i p}$ and $R(K)_{\mu \nu}{ }^{a}$ are identically satisfied for the composite gauge fields in (3.6). These results are generally in agreement with earlier off-shell results $[15,16,18-20]$ as well as with on-shell results [23, 24].

\section{Off-shell dimensional reduction: matter multiplets}

In this section we consider the off-shell reduction of three $4 D$ supermultiplets in a background of conformal supergravity: the vector multiplet, the tensor multiplet and the hypermultiplet. The strategy is the same as previously followed for the Weyl and the KaluzaKlein vector multiplet and the results turn out to be mutually consistent. At the end we note that there exists a second $3 D$ hypermultiplet that arises upon applying (3.3).

The vector multiplet. In four space-time dimensions the vector supermultiplet consists of a complex scalar $X$, a chiral spinor doublet $\Omega_{i}$, a gauge field $W_{M}$ and a triplet of auxiliary field $Y_{i j}$ which transform under Q- and S-supersymmetry transformations as follows,

$$
\begin{aligned}
\delta X & =\bar{\epsilon}^{i} \Omega_{i}, \\
\delta \Omega_{i} & =2 \not D X \epsilon_{i}+\frac{1}{2} \varepsilon_{i j}\left(F_{A B}^{-}-\frac{1}{4} \bar{X} T_{A B}{ }^{k l} \varepsilon_{k l}\right) \gamma^{A B} \epsilon^{j}+Y_{i j} \epsilon^{j}+2 X \eta_{i}, \\
\delta W_{M} & =\varepsilon^{i j} \bar{\epsilon}_{i}\left(\gamma_{M} \Omega_{j}+2 \psi_{M j} X\right)+\varepsilon_{i j} \bar{\epsilon}^{i}\left(\gamma_{M} \Omega^{j}+2 \psi_{M}{ }^{j} \bar{X}\right), \\
\delta Y_{i j} & =2 \bar{\epsilon}_{(i} \not D \Omega_{j}+2 \varepsilon_{i k} \varepsilon_{j l} \bar{\epsilon}^{(k} \not D \Omega^{l)},
\end{aligned}
$$


where $\left(Y^{i j}\right)^{*} \equiv Y_{i j}=\varepsilon_{i k} \varepsilon_{j l} Y^{k l}$, and $F_{M N}^{-}$denotes the anti-selfdual supersymmetrized component of the field strength $F_{M N}=\partial_{M} W_{N}-\partial_{N} W_{M}$. Under local scale and chiral transformations the fields transform according to the weights shown in table 3 .

Upon reduction to three dimensions, the vector field decomposes into a $3 D$ vector field $W_{\mu}$ and a scalar field $W=W_{\hat{4}}$ according to the standard Kaluza-Klein decomposition

$$
W_{M}=\left(\begin{array}{c}
W_{\mu}+B_{\mu} W \\
W
\end{array}\right) .
$$

As it will turn out, it is convenient to define the following linear combinations for the fermions and the auxiliary triplet,

$$
\begin{aligned}
\breve{\Omega}_{i}= & \Omega_{i}+\phi^{2} W \varepsilon_{i j} \psi^{j} \\
\breve{Y}_{i j}= & Y_{i j}+\phi^{2} W \varepsilon_{i k} \nu^{k}{ }_{j}+\phi^{3} W \bar{\psi}^{k} \gamma^{4} \psi_{(j} \varepsilon_{i) k}-\phi^{2} X \bar{\psi}_{(i} \psi_{j)}-\phi^{2} \bar{X} \varepsilon_{i k} \varepsilon_{j l} \bar{\psi}^{(k} \psi^{l)} \\
& -\phi \bar{\psi}_{(i} \gamma^{4} \breve{\Omega}_{j)}-\varepsilon_{i k} \varepsilon_{j l} \phi \bar{\psi}^{(k} \gamma^{4} \breve{\Omega}^{l)} .
\end{aligned}
$$

The supersymmetry variations then take the following form,

$$
\begin{aligned}
\delta X= & \bar{\epsilon}^{i} \breve{\Omega}_{i}-\frac{1}{2} \tilde{\Sigma}^{-} \phi W, \\
\delta(\phi W)= & \varepsilon^{i j} \bar{\epsilon}_{i} \gamma_{4} \breve{\Omega}_{j}+\varepsilon_{i j} \bar{\epsilon}^{i} \gamma_{4} \breve{\Omega}^{j}+\tilde{\Sigma}^{-} \bar{X}+\tilde{\Sigma}^{+} X, \\
\delta \breve{\Omega}_{i}= & 2 \not D\left(\mathcal{A}^{0}\right) X \epsilon_{i}+\phi W \mathcal{A}^{-} \epsilon_{i} \\
& +\varepsilon_{i j} \not D(\phi W) \gamma_{4} \epsilon^{i}-\left(X \mathcal{A}^{+}+\bar{X} \mathcal{A}^{-}\right) \gamma_{4} \varepsilon_{i j} \epsilon^{j} \\
& +\frac{1}{2} \varepsilon_{i j} \hat{F}(W)_{a b} \gamma^{a b} \epsilon^{j}+\breve{Y}_{i j} \epsilon^{j}+\mathrm{i} C\left(2 X \gamma_{4} \epsilon_{i}+\phi W \varepsilon_{i j} \epsilon^{j}\right) \\
& +2 X \eta_{i}-\phi W \gamma_{4} \varepsilon_{i j} \eta^{j}+\frac{1}{2} \tilde{\Sigma}^{+} \varepsilon_{i j} \gamma_{4} \breve{\Omega}^{j}, \\
\delta W_{\mu}= & \varepsilon^{i j} \bar{\epsilon}_{i}\left(\gamma_{\mu} \breve{\Omega}_{j}+2 \psi_{\mu j} X+\phi W \varepsilon_{j k} \gamma_{4} \psi_{\mu}{ }^{k}\right)+\text { h.c. }, \\
\delta \breve{Y}_{i j}= & 2 \bar{\epsilon}_{(i} \not D\left(\mathcal{A}^{0}\right) \breve{\Omega}_{j)}-\bar{\epsilon}^{k} \mathcal{A}^{+} \gamma_{4} \breve{\Omega}_{(i} \varepsilon_{j) k} \\
& +2 \mathrm{i} C \bar{\epsilon}_{(i} \gamma_{4} \breve{\Omega}_{j)}-\bar{\epsilon}_{(i}\left(2 X \breve{\chi}_{j)}+\phi W \varepsilon_{j) k} \breve{\chi}^{k}\right)-\eta_{(i} \breve{\Omega}_{j)}+\text { h.c. },
\end{aligned}
$$

where we have again suppressed the field-dependent S-supersymmetry and SU(2) transformations with parameters $\tilde{\eta}_{i}$ and $\tilde{\Lambda}_{i}{ }^{j}$ as in (2.17). In deriving the above result all higherorder terms in the fermions were taken into account, with the exception of those appearing in the variation of the auxiliary fields. The covariant derivative $D_{\mu}\left(\mathcal{A}^{0}\right)$ is $3 D$ Lorentz covariant and contains the modified U(1) gauge field defined in (2.16). The latter appears always in combination with terms proportional to $\mathcal{A}_{\mu}^{ \pm}$that will eventually provide the full $\mathrm{SU}(2)$ covariantization. Furthermore, we have used various expressions defined in (2.15), (2.17) and (2.21). Finally, note that the spinor $\breve{\chi}^{i}$ was defined in (2.21) and that in all the expressions we use the $3 D \mathrm{Q}$ - and S-supersymmetry gauge fields identified in section 2 .

The expressions found above can be compared to the expressions given for the KaluzaKlein multiplet, derived in the previous section. As it turns out, the $3 D$ supersymmetry 


\begin{tabular}{|c|c|c|c|c|c|c|c|c|c|c|}
\hline $4 D$ & \multicolumn{4}{|c|}{ vector multiplet } & \multicolumn{4}{|c|}{ tensor multiplet } & \multicolumn{2}{|c|}{ hypermultiplet } \\
\hline field & $X$ & $W_{\mu}$ & $\Omega_{i}$ & $Y^{i j}$ & $L^{i j}$ & $E_{\mu \nu}$ & $\varphi_{i}$ & $G$ & $A_{i}{ }^{\alpha}$ & $\zeta^{\alpha}$ \\
\hline$w$ & 1 & 0 & $\frac{3}{2}$ & 2 & 2 & 0 & $\frac{5}{2}$ & 3 & 1 & $\frac{3}{2}$ \\
\hline$c$ & -1 & 0 & $-\frac{1}{2}$ & 0 & 0 & 0 & $-\frac{1}{2}$ & 1 & 0 & $-\frac{1}{2}$ \\
\hline$\gamma_{5}$ & & & + & & & & - & & & - \\
\hline
\end{tabular}

Table 3. Weyl and chiral weights $\left(w\right.$ and $c$ ) and fermion chirality $\left(\gamma_{5}\right)$ of the vector multiplet, the tensor multiplet and the hypermultiplet component fields in four space-time dimensions.

transformations for the latter coincide with those given above upon introducing the appropriate redefinitions of fields in three dimensions,

$$
L_{q}^{p}=\Phi\left(\begin{array}{cc}
\mathrm{i} \phi W & 2 \mathrm{i} \bar{X} \\
2 \mathrm{i} X & -\mathrm{i} \phi W
\end{array}\right) \Phi^{-1}, \quad Y_{j}^{i}=\varepsilon^{i k} \breve{Y}_{k j}, \quad \Omega^{i p}=\Phi\left(\begin{array}{c}
-\gamma_{4} \breve{\Omega}^{j} \\
\varepsilon^{i j} \breve{\Omega}_{j}
\end{array}\right) .
$$

We can then write the supersymmetry variations (4.4) as

$$
\begin{aligned}
& \delta L^{p}{ }_{q}=2 \bar{\epsilon}_{i q} \Omega^{i p}-\delta^{p}{ }_{q} \bar{\epsilon}_{i r} \Omega^{i r}, \\
& \delta \Omega^{i p}=\not D L^{p}{ }_{q} \epsilon^{i q}-\frac{1}{2} F(W)_{a b} \gamma^{a b} \epsilon^{i p}+\hat{Y}_{j}^{i} \epsilon^{j p}+C L^{p}{ }_{q} \epsilon^{i q}+L^{p}{ }_{q} \eta^{i q}, \\
& \delta W_{\mu}=\bar{\epsilon}_{i p} \gamma_{\mu} \Omega^{i p}+L^{p}{ }_{q} \bar{\epsilon}_{i p} \psi_{\mu}{ }^{i q} \\
& \delta Y^{i}{ }_{j}=2 \bar{\epsilon}_{j p} \not D \Omega^{i p}-L^{p}{ }_{q} \bar{\epsilon}_{j p} \chi^{i q}-2 C \bar{\epsilon}_{j p} \Omega^{i p}-\bar{\eta}_{j p} \Omega^{i p}-(\text { trace }) .
\end{aligned}
$$

In these relations, we employ the $3 D$ gamma matrices defined in appendix $\mathrm{B}$ and the derivatives, $D_{\mu}$ are covariant with respect to all $3 D$ superconformal transformations including the emergent $\mathrm{SU}(2)_{\mathrm{R}}$, as in (2.42) with corresponding gauge fields defined according to (2.31). Note that the terms $\tilde{\Sigma}^{ \pm}$have been cancelled by the variation of the phase factor $\Phi$, just as before. The Weyl weights for the $3 D$ fields are given in table 4 .

The tensor multiplet. The tensor multiplet in four dimensions comprises an $\mathrm{SU}(2)$ triplet of scalars $L_{i j}$, a chiral spinor doublet $\varphi_{i}$, a two-form gauge field $E_{M N}$ and an auxiliary complex scalar field, $G$. The Weyl and chiral weights of these fields are summarized in table 3 . In a superconformal background the $Q$ - and $S$-supersymmetry transformations of the $4 D$ tensor supermultiplet fields take the following form,

$$
\begin{aligned}
\delta L_{i j} & =2 \bar{\epsilon}_{(i} \varphi_{j)}+2 \varepsilon_{i k} \varepsilon_{j l} \bar{\epsilon}^{(k} \varphi^{l)}, \\
\delta \varphi^{i} & =\not D L^{i j} \epsilon_{j}+\varepsilon^{i j} \hat{\epsilon} \epsilon_{j}-G \epsilon^{i}+2 L^{i j} \eta_{j}, \\
\delta G & =-2 \bar{\epsilon}_{i} \not D \varphi^{i}-\bar{\epsilon}_{i}\left(6 L^{i j} \chi_{j}+\frac{1}{4} \gamma^{A B} T_{A B j k} \varphi_{l} \varepsilon^{i j} \varepsilon^{k l}\right)+2 \bar{\eta}_{i} \varphi^{i}, \\
\delta E_{M N} & =\mathrm{i} \bar{\epsilon}^{i} \gamma_{M N} \varphi^{j} \varepsilon_{i j}-\mathrm{i} \bar{\epsilon}_{i} \gamma_{M N} \varphi_{j} \varepsilon^{i j}+2 \mathrm{i} L_{i j} \varepsilon^{j k} \bar{\epsilon}^{i} \gamma_{[M} \psi_{N] k}-2 \mathrm{i} L^{i j} \varepsilon_{j k} \bar{\epsilon}_{i} \gamma_{[M} \psi_{N]}^{k}, \\
\delta \hat{E}^{A} & =\varepsilon_{i j} \bar{\epsilon}^{i} \gamma^{A B} D_{B} \varphi^{j}+\frac{1}{4} \bar{\epsilon}^{i} \gamma^{A}\left(6 \varepsilon_{i j} \chi_{k} L^{j k}-\frac{1}{4} T_{B C i j} \gamma^{B C} \varepsilon^{j k} \varphi_{k}\right)+\frac{3}{2} \bar{\eta}^{i} \gamma^{A} \varphi^{j} \varepsilon_{i j}+\text { h.c. }
\end{aligned}
$$


Here, the derivatives $\mathcal{D}_{M}$ are covariant with respect to Lorentz transformations, dilatations and R-symmetry transformations. The vector, $\hat{E}^{M}$, denotes the superconformally covariant field strength

$$
\begin{aligned}
\hat{E}^{M}= & \frac{1}{2} \mathrm{i} e^{-1} \varepsilon^{M N P Q} \\
& \times\left[\partial_{N} E_{P Q}-\frac{1}{2} \mathrm{i} \bar{\psi}_{N}^{i} \gamma_{P Q} \varphi^{j} \varepsilon_{i j}+\frac{1}{2} \mathrm{i} \bar{\psi}_{N i} \gamma_{P Q} \varphi_{j} \varepsilon^{i j}-\mathrm{i} L_{i j} \varepsilon^{j k} \bar{\psi}_{N}{ }^{i} \gamma_{P} \psi_{Q k}\right],
\end{aligned}
$$

associated with the tensor field $E_{M N}$; the latter is subject to tensor gauge transformations parametrized by a vector $\lambda_{M}$,

$$
\delta E_{M N}=2 \partial_{[M} \lambda_{N]} .
$$

The Kaluza-Klein decomposition of the tensor gauge field reads

$$
E_{M N}=\left(\begin{array}{cc}
E_{\mu \nu}+2 B_{[\mu} E_{\nu]} & -E_{\mu} \\
E_{\nu} & 0
\end{array}\right),
$$

where $E_{\mu} \equiv E_{\hat{4} \mu}$. The tensor gauge transformation parameter decomposes accordingly into $\lambda_{M}=\left(\lambda_{\mu}, \lambda\right)$, where $\lambda \equiv \lambda_{\hat{4}}$, so that the $3 D$ tensor and vector fields, $E_{\mu \nu}$ and $E_{\mu}$ transform as

$$
\delta E_{\mu \nu}=2 \partial_{[\mu} \lambda_{\nu]}-2 B_{[\mu} \partial_{\nu]} \lambda, \quad \delta E_{\mu}=\partial_{\mu} \lambda .
$$

Let us now proceed and determine the reduction of the $4 D$ tensor field strength. First we note that $E_{A B C}=E_{A}{ }^{M} E_{B}{ }^{N} E_{C}{ }^{P} \partial_{[M} E_{N P]}$ decomposes as follows,

$$
\begin{aligned}
& E_{a b c}=e_{a}{ }^{\mu} e_{b}{ }^{\nu} e_{c}{ }^{\rho}\left(\partial_{[\mu} E_{\nu \rho]}+F(B)_{[\mu \nu} E_{\nu]}\right), \\
& E_{a b 4}=-\frac{1}{3} \phi F(E)_{a b} .
\end{aligned}
$$

where $F(E)_{\mu \nu}=\partial_{\mu} E_{\nu}-\partial_{\nu} E_{\mu}$. Note that $E_{a b c}$ and $E_{a b 4}$ have Weyl weight $w=3$, and they correspond to the bosonic part of the field strength (4.8) written with tangent-space indices. We can now write $E_{a b c}$ as a $3 D$ real scalar of weight $w=3$ by defining $E^{4} \equiv \frac{1}{2} \mathrm{i} \varepsilon^{a b c} E_{a b c}$. Indeed, a comparison with a two-rank tensor field in three dimensions shows that it represents only one degree of freedom (the $3 D$ tensor has three degrees of freedom from which one must subtract two gauge degrees of freedom). Hence, we can base ourselves exclusively on the scalar $E^{4}$ and ignore the underlying tensor field, without loss of generality. Therefore the field strength (4.8) written with tangent-space indices is precisely equal to

$$
\hat{E}^{4}=\frac{1}{2} \mathrm{i} \varepsilon^{a b c} E_{a b c}+\cdots, \quad \hat{E}^{a}=\frac{1}{2} \mathrm{i} \phi \varepsilon^{a b c} \hat{F}(E)_{b c}+\cdots
$$

where the dots denote the fermionic bilinears. Henceforth $\hat{E}^{4}$ will be regarded as a supercovariant scalar, as was explained above, whereas $\hat{F}(E)_{a b}$ denotes the supercovariant field strength associated with the $3 D$ gauge field $E_{\mu}$.

We are now ready to present the result of the reduction to three dimensions for the supersymmetry transformation rules. To this end, we find it useful to rescale the scalar 
triplet by $\phi$ to obtain a triplet of unit Weyl weight, and introduce the quantities

$$
\begin{aligned}
\breve{\varphi}_{i} & =\phi^{-1} \varphi_{i}+L_{i j} \gamma_{4} \psi^{j}, \\
\breve{E} & =\phi^{-1} \hat{E}^{4}+\frac{1}{2} \varepsilon_{i j} \mathcal{V}^{i}{ }_{k} L^{j k}-\varepsilon_{i j} \bar{\psi}^{i} \varphi^{j}-\varepsilon^{i j} \bar{\psi}_{i} \varphi_{j}-\frac{1}{2} \phi L^{i j} \bar{\psi}_{i} \gamma^{4} \psi^{k} \varepsilon_{j k}, \\
\breve{G} & =\phi^{-1} G-\bar{\psi}_{i} \gamma_{4} \varphi^{i}-\frac{1}{2} \phi L^{i j} \bar{\psi}_{i} \psi_{j} .
\end{aligned}
$$

With these definitions, we can write the reduced result as follows,

$$
\begin{aligned}
\delta\left(\phi^{-1} L_{i j}\right)= & 2 \bar{\epsilon}_{(i} \hat{\varphi}_{j)}+2 \varepsilon_{i k} \varepsilon_{j l} \bar{\epsilon}^{(k} \hat{\varphi}^{l)}, \\
\delta \breve{\varphi}^{i}= & \not D\left(\phi^{-1} L^{i j}\right) \epsilon_{j}+\frac{1}{2} \mathrm{i} e^{-1} \varepsilon^{\mu \nu \rho} F(E)_{\mu \nu} \gamma_{\rho} \varepsilon^{i j} \epsilon_{j}+\breve{E} \varepsilon^{i j} \gamma_{4} \epsilon_{j}-\breve{G} \epsilon^{i}, \\
& -\mathrm{i} \phi^{-1} L^{i j} C \gamma_{4} \epsilon_{j}+\phi^{-1} L^{i j} \eta_{j}+\frac{1}{2} \tilde{\Sigma}^{-} \varepsilon^{i j} \gamma_{4} \breve{\varphi}_{j}, \\
\delta E_{\mu}= & \mathrm{i} \varepsilon_{i j} \bar{\epsilon}^{i} \gamma_{\mu} \gamma_{4} \breve{\varphi}^{j}-\mathrm{i} \phi^{-1} L_{i j} \varepsilon^{j k} \bar{\epsilon}^{i} \gamma_{4} \psi_{\mu k}+\text { h.c. }, \\
\delta \breve{E}= & \varepsilon_{i j} \bar{\epsilon}^{i} \gamma_{4}\left(\not D\left(\mathcal{A}^{0}\right) \hat{\varphi}^{j}-\frac{1}{2} A^{+} \gamma_{4} \varepsilon^{j k} \breve{\varphi}_{k}\right)-\mathrm{i} C \varepsilon_{i j} \bar{\epsilon}^{i} \breve{\varphi}^{j}+\frac{1}{2} \varepsilon_{i j} \phi^{-1} L^{j k} \bar{\epsilon}^{i} \gamma_{4} \breve{\chi}_{k} \\
& +\frac{1}{2} \varepsilon_{i j} \bar{\varphi}^{i} \gamma_{4} \eta^{j}-\frac{1}{2} \tilde{\Sigma}^{+} \breve{G}+\text { h.c. }, \\
\delta \breve{G}= & -2 \bar{\epsilon}_{i}\left(\not D\left(\mathcal{A}^{0}\right) \breve{\varphi}^{i}-\frac{1}{2} A^{+} \gamma_{4} \varepsilon^{i j} \breve{\varphi}_{j}\right)+2 \mathrm{i} C \bar{\epsilon}_{i} \gamma_{4} \breve{\varphi}^{i}-\phi^{-1} L^{i j} \bar{\epsilon}_{i} \breve{\chi}_{j} \\
& +\bar{\eta}_{i} \breve{\varphi}^{i}+\tilde{\Sigma}^{-} \breve{E},
\end{aligned}
$$

where we retained all fermionic terms in the variations of $L_{i j}, \breve{\varphi}$ and $E_{\mu}$, but considered only the variations linear in the fermions for $\breve{G}$ and $\breve{E}$. In these expressions we used once again the covariant derivative $D_{\mu}\left(\mathcal{A}^{0}\right)$ that contains the modified $\mathrm{U}(1)$ gauge field in (2.16). We have again suppressed the field-dependent S-supersymmetry and $\mathrm{SU}(2)$ transformations in the above result.

To write the supersymmetry variations (4.15) in three-dimensional form, we employ a definition of fields that transform covariantly under the the local R-symmetry,

$$
L_{j}^{i}=\phi^{-1} \varepsilon^{i k} L_{k j}, \quad Y^{p}=\Phi\left(\begin{array}{cc}
\mathrm{i} \breve{E} & -\mathrm{i} \breve{G} \\
-\mathrm{i} \breve{G} & -\mathrm{i} \breve{E}
\end{array}\right) \Phi^{-1}, \quad \varphi^{i p}=\Phi\left(\begin{array}{c}
\mathrm{i} \breve{\varphi}^{i} \\
\mathrm{i} \gamma_{4} \varepsilon^{i j} \breve{\varphi}_{j}
\end{array}\right) .
$$

The supersymmetry variations resulting upon use of these definitions are as follows

$$
\begin{aligned}
\delta L^{i}{ }_{j} & =2 \bar{\epsilon}_{j p} \varphi^{i p}-\delta^{i}{ }_{j} \bar{\epsilon}_{k p} \varphi^{k p}, \\
\delta \varphi^{i p} & =\not D L^{i}{ }_{j} \epsilon^{j p}-\frac{1}{2} F(E)_{a b} \gamma^{a b} \epsilon^{i p}+Y^{p}{ }_{q} \epsilon^{i q}-C L^{i}{ }_{j} \epsilon^{j p}+L^{i}{ }_{j} \eta^{j p}, \\
\delta E_{\mu} & =\bar{\epsilon}_{i p} \gamma_{\mu} \varphi^{i p}+L^{i}{ }_{j} \bar{\epsilon}_{i p} \psi_{\mu}{ }^{j p} \\
\delta Y^{p}{ }_{q} & =2 \bar{\epsilon}_{i q} \not D \varphi^{i p}+L^{i}{ }_{j} \bar{\epsilon}_{j q} \chi^{i p}+2 C \bar{\epsilon}_{i q} \varphi^{i p}-\bar{\eta}_{i q} \varphi^{i p}-(\text { trace }) .
\end{aligned}
$$

Once again, the variations proportional to $\tilde{\Sigma}^{ \pm}$cancel. All the fields and the gamma matrices refer to $3 D$; the covariant derivative, $D_{\mu}$ also includes the gauge fields associated with the extra local $\mathrm{SU}(2)_{\mathrm{R}}$ symmetry, just as in (2.42). The Weyl weight of the component fields are summarized in table 4 . 


\begin{tabular}{|c|cccc|cccc|cc|}
\hline $3 D$ & \multicolumn{4}{|c|}{ vector multiplet } & \multicolumn{4}{c|}{ tensor multiplet } & \multicolumn{2}{c|}{ hypermultiplet } \\
\hline field & $L_{q}^{p}$ & $W_{\mu}$ & $\Omega^{i p}$ & $Y_{j}^{i}$ & $L_{j}^{i}$ & $E_{\mu}$ & $\varphi^{i p}$ & $Y_{q}^{p}$ & $A_{i}{ }^{\alpha}$ & $\zeta^{\alpha}$ \\
\hline$w$ & 1 & 0 & $\frac{3}{2}$ & 2 & 1 & 0 & $\frac{3}{2}$ & 2 & $\frac{1}{2}$ & 1 \\
\hline
\end{tabular}

Table 4. Matter multiplet fields with corresponding Weyl weights of the vector multiplet, the tensor multiplet and the hypermultiplet fields in three space-time dimensions.

Note that the supersymmetry transformations for the tensor multiplet (4.17) are very similar to those of the vector multiplet given in (4.6). In fact they are related precisely by the exchange symmetry noted for the Weyl multiplet in (3.3). We will return to this issue later in this section.

The hypermultiplet. The hypermultiplets are not realized off-shell, but they can be coupled to conformal supergravity provided the target-space geometry is restricted to a hyperkähler cone. For rigid supersymmetry it is sufficient that the target space is hyperkähler, but in order for the action to be superconformally invariant the target space must also admit a homothetic conformal Killing vector. This in turn implies that the homothetic Killing vector can locally be expressed in terms of the so-called hyperkähler potential which also defines the target-space metric $[11,26]$. Assuming that these conditions are met, let us now introduce the local Q- and S-supersymmetry transformations of the hypermultiplet fields, which only close modulo the equations of motion of the fermion fields,

$$
\begin{aligned}
\delta A_{i}{ }^{\alpha}+\delta \phi^{B} \Gamma_{B}{ }^{\alpha}{ }_{\beta} A_{i}{ }^{\beta} & =2 \bar{\epsilon}_{i} \zeta^{\alpha}+2 \varepsilon_{i j} G^{\alpha \bar{\beta}} \Omega_{\bar{\beta} \bar{\gamma}} \bar{\epsilon}^{j} \zeta^{\bar{\gamma}}, \\
\delta \zeta^{\alpha}+\delta \phi^{A} \Gamma_{A}{ }^{\alpha}{ }_{\beta} \zeta^{\beta} & =\not D A_{i}{ }^{\alpha} \epsilon^{i}+A_{i}{ }^{\alpha} \eta^{i}, \\
\delta \zeta^{\bar{\alpha}}+\delta \phi^{A} \bar{\Gamma}_{A}{ }^{\bar{\alpha}}{ }_{\bar{\beta}} \zeta^{\bar{\beta}} & =\not D A^{i \bar{\alpha}} \epsilon_{i}+A^{i \bar{\alpha}} \eta_{i} .
\end{aligned}
$$

where we employ the local sections of an $\operatorname{Sp}(r) \times \operatorname{Sp}(1)$ bundle, denoted by $A_{i}{ }^{\alpha}$, for $\alpha=$ $1,2, \ldots, 2 r$. The Weyl and chiral weights of these quantities are shown in table 3 . We also note the existence of a covariantly constant hermitian tensor $G^{\alpha \bar{\beta}}$ (which is used in raising and lowering indices) and of a covariantly constant skew-symmetric tensor $\Omega_{\alpha \beta}$ (and its complex conjugate $\bar{\Omega}^{\bar{\alpha}} \bar{\beta}$ satisfying $\Omega_{\bar{\alpha} \bar{\gamma}} \bar{\Omega}^{\bar{\beta}} \bar{\gamma}=-\delta_{\bar{\alpha}} \bar{\beta}$ ). Covariant derivatives contain the $\operatorname{Sp}(r)$ connection $\Gamma_{A}{ }_{\beta}{ }_{\beta}$, associated with rotations of the fermions. The sections $A_{i}{ }^{\alpha}$ are pseudo-real, i.e. they are subject to the constraint, $\varepsilon^{i j} \bar{\Omega}^{\bar{\alpha} \bar{\beta}} G_{\bar{\beta} \gamma} A_{i}^{\gamma}=A^{j \bar{\beta}} \equiv\left(A_{j}{ }^{\beta}\right)^{*}$. For our purpose the geometry of the hyperkähler cone is not relevant and we assume for simplicity that the cone is flat, so that the target-space connections and curvatures will vanish. The sections can then be identified with the fields, and the tensors $G^{\alpha \bar{\beta}}$ and $\Omega_{\alpha \beta}$ are constant $[25,26]$. The extension to non-trivial hyperkähler cone geometries is straightforward.

The Weyl and chiral weights of the sections and the fermion fields are listed in table 3. The $3 D$ hypermultiplet fields will be rescaled, however, so that they have the appropriate canonical dimension in $3 D$. This motivates the following field redefinitions,

$$
\begin{aligned}
\breve{A}_{i}{ }^{\alpha} & =\phi^{-1 / 2} A_{i}{ }^{\alpha}, \\
\breve{\zeta}^{\alpha} & =\phi^{-1 / 2} \zeta^{\alpha}+\frac{1}{2} \phi^{1 / 2} A_{j}{ }^{\alpha} \gamma^{4} \psi^{j}, \\
\breve{\zeta}^{\bar{\alpha}} & =\phi^{-1 / 2} \zeta^{\bar{\alpha}}+\frac{1}{2} \phi^{1 / 2} A^{i \bar{\alpha}} \gamma^{4} \psi_{i} .
\end{aligned}
$$


Upon dimensional reduction the supersymmetry transformation rules (4.18) can be cast in the form,

$$
\begin{aligned}
\delta \breve{A}_{i}{ }^{\alpha} & =2 \bar{\epsilon}_{i} \breve{\zeta}^{\alpha}+2 \varepsilon_{i j} G^{\alpha \bar{\beta}} \Omega_{\bar{\beta} \bar{\gamma}} \bar{\epsilon}^{j} \breve{\zeta}^{\bar{\gamma}} \\
\delta \breve{\zeta}^{\alpha} & =\not D \breve{A}_{i}{ }^{\alpha} \epsilon^{i}+\frac{1}{2} \mathrm{i} C \breve{A}_{i}{ }^{\alpha} \gamma_{4} \epsilon^{i}+\frac{1}{2} \tilde{\Sigma}^{-} G^{\alpha \bar{\beta}} \Omega_{\bar{\beta} \bar{\gamma}} \gamma_{4} \breve{\zeta}^{\bar{\gamma}}+\frac{1}{2} \breve{A}_{i}{ }^{\alpha} \eta^{i}
\end{aligned}
$$

where all fermionic terms were taken into account and where $D_{\mu}$ denotes the supercovariant derivative in three dimensions. Again we suppressed the field-dependent SU(2) and Ssupersymmetry transformations. Subsequently we further redefine the fermion such as to incorporate their consistent transformation behaviour under local R-symmetry,

$$
\zeta^{\alpha p}=\Phi\left(\begin{array}{c}
-\mathrm{i} G^{\alpha \bar{\beta}} \Omega_{\bar{\beta} \bar{\gamma} \bar{\zeta}} \breve{\zeta}^{\bar{\gamma}} \\
\mathrm{i} \gamma_{4} \breve{\zeta}^{\alpha}
\end{array}\right) .
$$

The Weyl weights of the $3 D$ quantities $A_{i}{ }^{\alpha}$ and $\zeta^{\alpha}$ have been shown in table 4 . With these redefinitions we obtain the following $3 D$ supersymmetry transformations of the $3 D$ fields,

$$
\begin{aligned}
\delta A_{i}{ }^{\alpha} & =2 \bar{\epsilon}_{i p} \zeta^{\alpha p}, \\
\delta \zeta^{\alpha p} & =\not D A_{i}{ }^{\alpha} \epsilon^{i p}-\frac{1}{2} C A_{i}{ }^{\alpha} \epsilon^{i p}+\frac{1}{2} A_{i}{ }^{\alpha} \eta^{i p},
\end{aligned}
$$

expressed in terms of $3 D$ gamma matrices and supercovariant derivatives. The terms proportional to $\tilde{\Sigma}^{ \pm}$have again disappeared as they should.

As we alluded to at the beginning of the section, there is an alternative hypermultiplet that transforms under the other $\mathrm{SU}(2)$ factor of the R-symmetry, which cannot emerge under dimensional reduction. For future reference we give its transformation rules below, relying on the reflection (3.3),

$$
\begin{aligned}
\delta \tilde{A}_{p}^{\alpha} & =2 \bar{\epsilon}_{i p} \tilde{\zeta}^{\alpha i} \\
\delta \tilde{\zeta}^{\alpha i} & =\not D \tilde{A}_{p}^{\alpha} \epsilon^{i p}+\frac{1}{2} C \tilde{A}_{p}^{\alpha} \epsilon^{i p}+\frac{1}{2} \tilde{A}_{p}^{\alpha} \eta^{i p} .
\end{aligned}
$$

\section{Four and three-dimensional fields and invariant Lagrangians}

In this section we express the $4 D$ fields in terms of the $3 D$ ones; subsequently we convert the known supersymmetric $4 D$ Lagrangians by direct substitution in terms of the supersymmetric $3 D$ Lagrangians. The section is divided into three subsections. In the first one we express the $4 D$ bosonic fields in terms of the $3 D$ ones that were identified in the previous sections based on the off-shell supersymmetry transformations. The fermionic fields are ignored, as the supersymmetry transformations are fully known in both $3 D$ and $4 D$. In the second subsection we consider three $4 D$ supersymmetric Lagrangians quadratic in derivatives and derive the corresponding expressions for the bosonic terms belonging to the reduced $3 D$ Lagrangians. Because the reduction procedure is fully off-shell (with the exception of the hypermultiplets that are not defined as genuine off-shell multiplets) there is no need for additional adjustments. In the third subsection we discuss some features of the c-map and compare to results in the literature. 


\subsection{The supercovariant dictionary: expressing $4 D$ fields in terms of $3 D$ fields}

Some of the $4 D$ and $3 D$ fields are identical, except that the latter will no longer depend on the fourth coordinate. For instance, the vierbein fields, which contain the three-dimensional fields $e_{\mu}{ }^{a}$ as a submatrix, belong to this class. For other fields the relation is more involved. In particular for the Kaluza-Klein scalar $\phi$, matters are more subtle, as this field is contained in a $2 \times 2$ anti-hermitian traceless matrix $L_{q}^{p}{ }_{q}^{0}$, defined in (2.34) by absorbing a phase factor $\Phi$, introduced in subsection 2.3 to realize the new local $\mathrm{SU}(2)$ factor of the $3 D$ R-symmetry group. As a result the expressions for the $4 D$ fields are invariant under the new local $\mathrm{SU}(2)$ R-symmetry that emerges in the reduction, up to a term that takes the form of a $4 D \mathrm{U}(1)$ R-symmetry.

The $3 D$ vector and tensor matter supermultiplets contain scalar fields that were conveniently written in terms of anti-hermitian traceless $2 \times 2$ matrices. For instance, the Kaluza-Klein multiplet contains the scalar $L_{q}^{p}$ with Weyl weight $w=1$, as well as a similar field $Y_{j}^{i}{ }_{j}^{0}$ of weight $w=2$. The vector multiplets corresponding to the matter $4 D$ vector supermultiplets also contain these fields, $L^{p}{ }_{q}$ and $Y^{i}{ }_{j}$, which depend linearly on the components of the $4 D$ vector multiplet. The same situation arises for the tensor multiplet, but with the indices $p, q$ and $i, j$ interchanged. Hence these multiplets contain fields $L^{i}{ }_{j}$ and $Y^{p}$, with Weyl weights $w=1$ and $w=2$, respectively. Obviously, in the context of Lagrangians that are at most quadratic in derivatives, the fields $L$ correspond to the physical scalars and the fields $Y$ to the auxiliary fields.

Although the matrix form of the scalar is convenient when considering the supersymmetry transformations, it is not always easy to write the results in the form of matrix products and traces thereof. Therefore we will use a uniform decomposition in terms of the three independent components transforming as a vector under the appropriate $\mathrm{SU}(2)$ R-symmetry group. Hence for the vector multiplet we have

$$
L_{q}^{p}(x, v, \bar{v})=\left(\begin{array}{cc}
-\frac{1}{2} \mathrm{i} x & v \\
-\bar{v} & \frac{1}{2} \mathrm{i} x
\end{array}\right), \quad Y_{j}^{i}(y, w, \bar{w})=\left(\begin{array}{cc}
-\frac{1}{2} \mathrm{i} y & w \\
-\bar{w} & \frac{1}{2} \mathrm{i} y
\end{array}\right)
$$

and for the tensor multiplet we have corresponding definitions for $L^{i}{ }_{j}(x, v, \bar{v})$ and $Y^{p}{ }_{q}(y, w, \bar{w})$. Obviously, in Lagrangians with both vector and tensor multiplets, these multiplets should in principle be labelled by different indices. We recall the components of $\mathcal{D}_{\mu} L^{p}{ }_{q}$ and $\mathcal{D}_{\mu} L^{i}{ }_{j}$ for convenience,

$$
\begin{aligned}
& \mathcal{D}_{\mu} L^{p}{ }_{q}=\left(\partial_{\mu}-b_{\mu}\right) L^{p}{ }_{q}+\frac{1}{2}\left[\mathcal{A}_{\mu}, L\right]^{p}, \\
& \mathcal{D}_{\mu} L^{i}{ }_{j}=\left(\partial_{\mu}-b_{\mu}\right) L^{i}{ }_{j}+\frac{1}{2}\left[\mathcal{V}_{\mu}, L\right]_{j}^{i},
\end{aligned}
$$

which is in agreement with (2.38).

Let us now consider the $4 D$ Weyl multiplet, whose fields can be expressed in terms of the fields of the $3 D$ Weyl multiplet and the Kaluza-Klein vector multiplet (restricting ourselves to the bosonic fields and ignoring that the redefined bosonic fields may also 
contain fermionic bilinears),

$$
\begin{aligned}
& e_{M}^{A}=\left\{\begin{array}{l}
e_{\mu}{ }^{a}=e_{\mu}{ }^{a} \\
e_{\mu}{ }^{4}=W_{\mu}{ }^{0}\left(L^{0}\right)^{-1} \\
e_{\hat{4}}{ }^{a}=0 \\
e_{\hat{4}}{ }^{4}=\left(L^{0}\right)^{-1}
\end{array}\right. \\
& L^{0}=\sqrt{\left|v^{0}\right|^{2}+\frac{1}{4}\left(x^{0}\right)^{2}}=\sqrt{-\frac{1}{2} L_{q}^{p}{ }_{q}^{0} L_{p}^{q}}, \\
& B_{\mu}=W_{\mu}^{0} \text {, } \\
& \mathcal{V}_{\left.M^{i}{ }_{j}\right|_{4 D}}=\left\{\begin{array}{l}
\mathcal{V}_{\mu}{ }^{i}{ }_{j}=\mathcal{V}_{\mu}{ }^{i}{ }_{j}+W_{\mu}{ }^{0} Y^{i}{ }_{j}{ }^{0}\left(L^{0}\right)^{-2}, \\
\mathcal{V}_{\hat{4} i}{ }^{i}{ }_{j}=Y^{i}{ }_{j}^{0}\left(L^{0}\right)^{-2},
\end{array}\right. \\
& A_{M}=\left\{\begin{array}{l}
A_{\mu}=\mathcal{A}_{\mu}{ }^{0}+\frac{1}{L^{0}}\left[\frac{1}{4} F\left(W^{0}\right)_{\mu}+W_{\mu}{ }^{0} C\right], \\
A_{\hat{4}}=\left(L^{0}\right)^{-1} C,
\end{array}\right. \\
& T_{A B}{ }^{i j}=\left\{\begin{array}{l}
T_{a b}{ }^{i j}=\frac{1}{2} \mathrm{i}\left(L^{0}\right)^{-2} \varepsilon^{i j} \varepsilon_{a b c}\left[\left(\bar{v}^{0} \overleftrightarrow{\mathcal{D}}^{c} x^{0}\right)-\frac{\bar{v}^{0}}{L^{0}+\frac{1}{2} x^{0}}\left(v^{0} \overleftrightarrow{\mathcal{D}}^{c} \bar{v}^{0}\right)\right], \\
T_{a 4}{ }^{i j}=\frac{1}{2} \mathrm{i}\left(L^{0}\right)^{-2} \varepsilon^{i j}\left[\left(\bar{v}^{0} \overleftrightarrow{\mathcal{D}}_{a} x^{0}\right)-\frac{\bar{v}^{0}}{L^{0}+\frac{1}{2} x^{0}}\left(v^{0} \overleftrightarrow{\mathcal{D}}_{a} \bar{v}^{0}\right)\right],
\end{array}\right. \\
& \left.D\right|_{4 D}=\frac{1}{2} D-\frac{1}{12} R-\frac{1}{3}\left(L^{0}\right)^{-1} \mathcal{D}^{a} \mathcal{D}_{a} L^{0}+\frac{1}{6}\left(L^{0}\right)^{-2}\left(\mathcal{D}_{a} L^{0}\right)^{2} \\
& -\frac{1}{12}\left(L^{0}\right)^{-2} F\left(W^{0}\right)_{a b}{ }^{2}-\frac{1}{2} C^{2}-\frac{1}{4}\left(L^{0}\right)^{-2} Y^{i}{ }_{j}^{0} Y_{i}^{j}{ }_{i}^{0},
\end{aligned}
$$

where the covariant derivatives contain the connections $\mathcal{A}_{\mu}{ }^{p} q$ associated with the second $3 D$ $\mathrm{SU}(2) \mathrm{R}$-symmetry group and $R$ denotes the three-dimensional Ricci scalar. As explained in section 2 the fields $L^{p}{ }^{0}(x, v, \bar{v}), W_{\mu}{ }^{0}$ and $Y^{i}{ }_{j}{ }^{0}$ denote the bosonic fields of the KaluzaKlein supermultiplet. The remaining fields belong to the $3 D$ Weyl multiplet and were discussed in section 3. The connection $\mathcal{A}_{\mu}{ }^{0}$ was defined in the second equation of (2.48). Its explicit form is not relevant, but it is important to realize that under local $3 D \mathrm{SU}(2)$ it transforms as the connection of the $4 D \mathrm{U}(1) \mathrm{R}$-symmetry.

Subsequently we consider the $4 D$ vector multiplet, which upon reduction leads to a $3 D$ vector multiplet. The bosonic fields of the latter are denoted by $L^{p}{ }_{q}(x, v, \bar{v}), W_{\mu}$ and $Y^{i}{ }_{j}$,

$$
\begin{aligned}
& X=-\frac{1}{4} \mathrm{i}\left[\frac{x \bar{v}^{0}-\bar{v} x^{0}}{L^{0}}-\frac{\bar{v} v^{0}-v \bar{v}^{0}}{L^{0}\left(L^{0}+\frac{1}{2} x^{0}\right)} \bar{v}^{0}\right], \\
& W_{M}=\left\{\begin{array}{l}
W_{\mu}=W_{\mu}+W_{\mu}^{0} \frac{L^{p}{ }_{q} L_{p}^{0}}{2\left(L^{0}\right)^{2}}, \\
W_{\hat{4}}=\frac{L_{q}^{p} L^{q} p^{0}}{2\left(L^{0}\right)^{2}},
\end{array}\right. \\
& Y_{i j}=-\varepsilon_{i k}\left[Y^{k}{ }_{j}+Y_{j}^{k}{ }_{j}^{0} \frac{L_{q}^{p} L_{p}^{q}{ }_{p}^{0}}{2\left(L^{0}\right)^{2}}\right] \text {, }
\end{aligned}
$$

where we note that

$$
L_{q}^{p} L_{p}^{q}{ }_{p}^{0}=-\frac{1}{2} x x^{0}-v \bar{v}^{0}-\bar{v} v^{0}
$$


For the $3 D$ vector multiplet the following transformations will define an invariance,

$$
\begin{aligned}
& \delta L^{p}{ }_{q}=\alpha L^{p}{ }^{0}, \quad \delta L^{p}{ }^{0}=0, \\
& \delta W_{\mu}=\alpha W_{\mu}^{0}, \quad \delta W_{\mu}{ }^{0}=0, \\
& \delta Y^{i}{ }_{j}=\alpha Y^{i}{ }_{j}{ }^{0}, \quad \delta Y^{i}{ }^{0}{ }^{0}=0,
\end{aligned}
$$

where $\alpha$ is constant parameter, because the $4 D$ vector components remain invariant under (5.6), with the exception of $W_{\hat{4}}$. The latter is shifted by a constant which represents a remnant of the full $4 D$ gauge transformations. This shows that (5.6) defines an invariance for any $4 D$ locally supersymmetric Lagrangian that involves vector multiplets upon dimensional reduction. In principle there are additional invariances, as discussed in [3], but those are not immediately relevant for what follows. The tensor multiplet and the hypermultiplet do not give rise to symmetries such as (5.6).

The $4 D$ tensor multiplet reduces to the $3 D$ tensor multiplet. The bosonic fields of the latter are $L_{j}^{i}, E_{\mu}$ and $Y^{p}{ }_{q}(y, w, \bar{w})$,

$$
\begin{aligned}
L_{i j} & =-\varepsilon_{i k} L^{k}{ }_{j} L^{0}, \\
E^{A} & =\left\{\begin{array}{l}
E^{a}=\frac{1}{2} \mathrm{i} L^{0} \varepsilon^{a b c} F(E)_{b c}, \\
E^{4}=\frac{1}{2} Y^{p}{ }_{q} L^{q}{ }_{p}^{0}+\frac{1}{2} L^{i}{ }_{j} Y^{j}{ }_{i}^{0},
\end{array}\right. \\
E_{\hat{4} \mu} & =E_{\mu}, \\
G & =\frac{1}{2} \mathrm{i}\left[-y v^{0}+w x^{0}-\frac{\bar{w} v^{0}-w \bar{v}^{0}}{L^{0}+\frac{1}{2} x^{0}} v^{0}\right],
\end{aligned}
$$

We note an alternative expression for the scalar $X$ of the $4 D$ vector multiplet and a corresponding one for the auxiliary scalar $\bar{G}$ of the tensor multiplet,

$$
\begin{aligned}
& X=\frac{1}{2} \mathrm{i} \bar{v}-\frac{1}{4} \mathrm{i}\left[x-\frac{L^{p}{ }_{q} L^{q} p^{0}}{L^{0}}\right] \frac{\bar{v}^{0}}{L^{0}+\frac{1}{2} x^{0}}, \\
& \bar{G}=2 L^{0}\left[\frac{1}{2} \mathrm{i} \bar{w}-\frac{1}{4} \mathrm{i}\left[y-\frac{Y^{p}{ }_{q} L_{p}^{q} p^{0}}{L^{0}}\right] \frac{\bar{v}^{0}}{L^{0}+\frac{1}{2} x^{0}}\right],
\end{aligned}
$$

which will turn out to be useful later on.

Finally we consider the $4 D$ hypermultiplet which reduces to a $3 D$ hypermultiplet, where we have only a single bosonic quantity represented by the local sections $A_{i}{ }^{\alpha}$. Upon the reduction these sections are redefined according to

$$
\left.A_{i}{ }^{\alpha}\right|_{4 D}=\left(L^{0}\right)^{1 / 2} A_{i}{ }^{\alpha} .
$$

This completes the dictionary between the $4 D$ and $3 D$ fields. The reader may now verify explicitly that under an SU(2) R-symmetry transformation of the $3 D$ fields, the $4 D$ fields remain invariant up to a $4 D \mathrm{U}(1) \mathrm{R}$-symmetry transformation. This is guaranteed by the relations discussed in subsection 2.3 and more in particular by the equations (2.44)-(2.49). 


\subsection{Lagrangians quadratic in derivatives}

We now turn to the $4 D$ Lagrangians quadratic in space-time derivatives and reduce them to three dimensions in terms of the $3 D$ fields that we have derived. We will restrict ourselves to the bosonic expressions, because supersymmetry is ensured in the off-shell reduction. We start with the hypermultiplet Lagrangian, because that is the simplest one. Subsequently we will discuss the tensor multiplet Lagrangian and finally the vector Lagrangian.

\subsubsection{The hypermultiplet Lagrangian}

The $4 D$ bosonic Lagrangian for hypermultiplets reads [26],

$$
\left.\mathcal{L}_{\text {hyper }}\right|_{4 D}=-\frac{1}{2} E \Omega_{\alpha \beta} \varepsilon^{i j}\left[\mathcal{D}_{M} A_{i}{ }^{\alpha} \mathcal{D}^{M} A_{j}{ }^{\beta}-A_{i}{ }^{\alpha} A_{j}{ }^{\beta}\left[\frac{1}{6} R+\frac{1}{2} D\right]\right] .
$$

Upon reduction to three dimensions, the first term becomes

$$
\begin{aligned}
-\frac{1}{2} E & \Omega_{\alpha \beta} \varepsilon^{i j} \mathcal{D}_{M} A_{i}{ }^{\alpha} \mathcal{D}^{M} A_{j}{ }^{\beta}=-\frac{1}{2} e \Omega_{\alpha \beta} \varepsilon^{i j} \\
& \times\left[\mathcal{D}_{\mu} A_{i}{ }^{\alpha} \mathcal{D}^{\mu} A_{j}{ }^{\beta}+A_{i}{ }^{\alpha} A_{j}{ }^{\beta}\left[-\frac{1}{2} \phi^{-1} \mathcal{D}^{\mu} \mathcal{D}_{\mu} \phi+\frac{3}{4} \phi^{-2}\left(\mathcal{D}_{\mu} \phi\right)^{2}-\frac{1}{8} \phi^{-2} Y^{i}{ }_{j}^{0} Y^{j}{ }_{i}^{0}\right]\right]
\end{aligned}
$$

where we suppressed a total derivative. Note that the covariant derivatives $\mathcal{D}_{\mu} A_{i}{ }^{\alpha}$ on the right-hand side contain the $3 D \mathrm{SU}(2)$ gauge fields $\mathcal{V}_{\mu}{ }^{i}{ }_{j}$. Next we turn to the second term in (5.10). Making use of (A.3), which relates the $4 D$ and $3 D$ Ricci scalars, and of (5.3), which gives the relation between the $4 D$ and $3 D D$-fields, the two terms readily combine into the $3 D$ Lagrangian,

$$
\left.e^{-1} \mathcal{L}_{\text {hyper }}\right|_{3 D}=-\frac{1}{2} \Omega_{\alpha \beta} \varepsilon^{i j}\left[\mathcal{D}_{\mu} A_{i}{ }^{\alpha} \mathcal{D}^{\mu} A_{j}{ }^{\beta}-\frac{1}{4} A_{i}{ }^{\alpha} A_{j}{ }^{\beta}\left(\frac{1}{2} R+D-C^{2}\right)\right],
$$

which agrees with the expression given in [16]. Observe that all the components of the Kaluza-Klein vector multiplet decouple from the hypermultiplet Lagrangian, so that the well-known property that vector multiplets and hypermultiplets have no direct interaction in the ungauged case, is preserved under the reduction. We will return to this feature in due course.

Of course, there exists a second $3 D$ hypermultiplet Lagrangian, which is obtained from applying the reflection symmetry (3.3). We have already given its transformation rules in (4.23). The corresponding Lagrangian takes the form

$$
\left.e^{-1} \tilde{\mathcal{L}}_{\text {hyper }}\right|_{3 D}=-\frac{1}{2} \tilde{\Omega}_{\alpha \beta} \varepsilon^{p q}\left[\mathcal{D}_{\mu} \tilde{A}_{p}{ }^{\alpha} \mathcal{D}^{\mu} \tilde{A}_{q}{ }^{\beta}-\frac{1}{4} \tilde{A}_{p}{ }^{\alpha} \tilde{A}_{q}{ }^{\beta}\left(\frac{1}{2} R-D-C^{2}\right)\right] .
$$

Such a Lagrangian can only be obtained from $4 D$ upon reducing a vector multiplet and applying $3 D$ vector-scalar duality. However, as we shall see in subsection 5.2.3, these hypermultiplet Lagrangians will belong to a restricted class. 


\subsubsection{The tensor multiplet Lagrangian}

Here we consider the tensor multiplet Lagrangian in four space-time dimensions, which reads as (we follow the notation of [5]),

$$
\begin{aligned}
\left.\mathcal{L}_{\text {tensor }}\right|_{4 D}= & -\frac{1}{2} E F(L)_{I J} \mathcal{D}_{M} L_{i j}{ }^{I} \mathcal{D}^{M} L^{i j J}+F(L)_{I J} L_{i j}{ }^{I} L^{i j J}\left(\frac{1}{3} R+D\right) \\
& +E F(L)_{I J}\left(E_{M}{ }^{I} E^{M J}-E^{M I} \mathcal{V}_{M}{ }^{i}{ }_{j} L_{i k}{ }^{I} \varepsilon^{j k}+G^{I} \bar{G}^{J}\right) \\
& +\frac{1}{2} \mathrm{i} \varepsilon^{M N P Q} F(L)_{I J K}{ }^{i j} E_{M N}{ }^{I} \partial_{P} L_{i k}{ }^{J} \partial_{Q} L_{j l}{ }^{K} \varepsilon^{k l},
\end{aligned}
$$

where the tensor multiplets have been labelled with indices $I, J, \ldots$ Here the functions $F_{I J}(L)$ depend on the tensor multiplet scalars $L_{i j}{ }^{I}$ and are invariant under the $\mathrm{SU}(2) \mathrm{R}$ symmetry group and homogeneous of degree -1 . Furthermore $F_{I J K}{ }^{i j}$ denotes the derivative of $F_{I J}$ with respect to $L_{i j}{ }^{K}$. The $E^{M I}$ are the bosonic field strengths associated with the tensor fields $E_{M N}{ }^{I}$, which follow from (4.8).

For any $4 D$ rigidly or locally supersymmetric tensor multiplet Lagrangian, the functions $F_{I J}(L)$ must satisfy the following equations [5, 27],

$$
F_{I J K}{ }^{i j}=F_{(I J K)}{ }^{i j}, \quad F_{I J K L}{ }^{i[j k] l}=0 .
$$

These conditions suffice to prove that there must exist a function $F(x, v, \bar{v})$ such that

$$
F_{I J}=\frac{\partial^{2} F(x, v, \bar{v})}{\partial x^{I} \partial x^{J}}=-\frac{\partial^{2} F(x, v, \bar{v})}{\partial v^{I} \partial \bar{v}^{J}}, \quad \frac{\partial^{2} F(x, v, \bar{v})}{\partial x^{I} \partial v^{J}}=\frac{\partial^{2} F(x, v, \bar{v})}{\partial x^{J} \partial v^{I}},
$$

where we have used $L^{21 I}=\frac{1}{2} \mathrm{i} x^{I}$ and $L^{11 I}=v^{I}$, which is consistent with earlier definitions. For superconformally invariant Lagrangians (as well as all locally supersymmetric Lagrangians) the function $F(x, v, \bar{v})$ can be chosen to be homogeneous of first degree and invariant under phase transformations of the components $v^{I}$ and $\bar{v}^{I}[11]$, so that

$$
\begin{aligned}
& x^{I} \frac{\partial F(x, v, \bar{v})}{\partial x^{I}}+ v^{I} \frac{\partial F(x, v, \bar{v})}{\partial v^{I}}+\bar{v}^{I} \frac{\partial F(x, v, \bar{v})}{\partial v^{I}}=F(x, v, \bar{v}), \\
& v^{I} \frac{\partial F(x, v, \bar{v})}{\partial v^{I}}-\bar{v}^{I} \frac{\partial F(x, v, \bar{v})}{\partial v^{I}}=0 .
\end{aligned}
$$

The SU(2) invariance and the homogeneity of the functions $F_{I J}(L)$ imply the equation

$$
F_{I J K}{ }^{i k} L_{k j}{ }^{K}=-\frac{1}{2} \delta^{i}{ }_{j} F_{I J},
$$

Unlike the functions $F_{I J}$, the function $F(x, v, \bar{v})$ is not invariant under the full $\mathrm{SU}(2)$ R-symmetry group, but only under a U(1) subgroup.

In the superconformal case it is convenient to introduce also an $\mathrm{SU}(2)$ invariant quantity whose second derivative generates the metric of the tensor multiplet scalars,

$$
\chi_{\text {tensor }}(L) \equiv 2 F_{I J} L^{i j I} L_{i j}{ }^{J},
$$

which is a homogeneous function of first degree invariant under $\mathrm{SU}(2)$. This tensorpotential $\chi_{\text {tensor }}$ satisfies the following equations,

$$
\frac{\partial \chi_{\text {tensor }}(L)}{\partial L_{i j}{ }^{I}}=2 F_{I J}(L) L^{i j J}, \quad \varepsilon_{k l} \frac{\partial^{2} \chi_{\text {tensor }}(L)}{\partial L_{i k}{ }^{I} \partial L_{j l}{ }^{J}}=2 F_{I J}(L) \varepsilon^{i j} .
$$


Not surprisingly there exists a relation between $\chi_{\text {tensor }}(L)$ and the function $F(x, v, \bar{v})$,

$$
\chi_{\text {tensor }}(L)=F_{I J}\left(x^{I} x^{J}+4 v^{I} \bar{v}^{J}\right)=-F(v, \bar{v}, x)+x^{I} \frac{\partial F(x, v, \bar{v})}{\partial x^{I}},
$$

which can be established by making use of the equations (5.15) and (5.16). The right-hand side of (5.20) coincides with the expression for the hyperkähler potential $\chi_{\text {hyper }}(v, \bar{v}, w, \bar{w})$ given in [11] for the hyperkähler cones that one obtains upon dualizing the tensor fields to scalars. In that case $\partial F(x, v, \bar{v}) / \partial x^{I}$ is identified with $w^{I}+\bar{w}^{I}$, so that one is performing a Legendre transformation [5]. The reason that only the real part of $w^{I}$ appears is that the hypermultiplet Lagrangian will have an abelian isometry for every tensor multiplet.

Observe that the last term in (5.13) specifies a coupling of the tensor gauge fields to $F_{I J K}{ }^{i j}$, the derivative of $F_{I J}$ with respect to $L_{i j}{ }^{K}$. This coupling does not involve the tensor field strengths, but is nevertheless invariant under tensor gauge transformations. The reason is that the term $F_{I J K}{ }^{i j} \partial_{P} L_{i k}{ }^{J} \partial_{Q} L_{j l}{ }^{K} \varepsilon^{k l}$ satisfies the equation,

$$
\partial_{[M}\left(F_{I J K}{ }^{i j} \partial_{P} L_{i k}^{J} \partial_{Q]} L_{j l}^{K} \varepsilon^{k l}\right)=0,
$$

by virtue of the properties satisfied by $F_{I J}$. This result implies that one can write (locally)

$$
F_{I J K}{ }^{i j} \partial_{P} L_{i k}^{J} \partial_{Q} L_{j l}^{K} \varepsilon^{k l}=\partial_{[P} A(x, v, \bar{v})_{Q] I} .
$$

One particular solution for the space-time vectors $A(x, v, \bar{v})_{M I}$ is defined in terms of second derivatives of the function $F(x, v, \bar{v})$ introduced in (5.15), and reads

$$
A(x, v, \bar{v})_{M I}=\frac{\partial^{2} F(x, v, \bar{v})}{\partial x^{I} \partial \bar{v}^{J}} \partial_{M} \bar{v}^{J}-\frac{\partial^{2} F(x, v, \bar{v})}{\partial x^{I} \partial v^{J}} \partial_{M} v^{J}
$$

Clearly these space-time vectors are only determined up to a gauge transformation $A_{M I} \rightarrow$ $A_{M I}+\partial_{M} \Lambda_{I}$. They are, however, only manifestly invariant under a (rigid) U(1) subgroup of the full SU(2) R-symmetry transformations. Nevertheless, when applying an infinitesimal $\mathrm{SU}(2)$ transformation with (local) parameter $\Lambda_{i}^{j}(x)$ on the left-hand side of (5.22), one obtains

$$
\delta_{\mathrm{SU}(2)}\left(F_{I J K}{ }^{i j} \partial_{P} L_{i k}^{J} \partial_{Q} L_{j l}{ }^{K} \varepsilon^{k l}\right)=\partial_{[P}\left[\partial_{Q]} \Lambda_{i}^{j} F_{I J}(L) L_{j k}{ }^{J} \varepsilon^{k i}\right],
$$

where we made use of the $\mathrm{SU}(2)$ invariance of $F_{I J}(L)$. This result, which is in line with (5.21), implies that the vectors $A(x, v, \bar{v})_{M I}$ are invariant under (rigid) SU(2) up to an abelian gauge transformation with field-dependent parameter. For the solution (5.23) one can calculate this transformation explicitly in terms of multiple derivatives of the function $F(x, v, \bar{v})$.

A relevant question is whether it is possible to apply such a gauge transformation to the $3 D$ vector fields $A(x, v, \bar{v})_{M I}$ such that the results become exactly $\mathrm{SU}(2)$ invariant. As was observed long ago [29], the answer to this question is in general negative: it is not always possible to satisfy (5.22) with $\mathrm{SU}(2)$ invariant 'potentials' $A(x, v, \bar{v})_{M I}$. However, as we will establish in the next subsection, there do exist specific models where both the gauge invariance and the $\mathrm{SU}(2)$ invariance is manifest. Hence we may distinguish two distinct classes of tensor interactions characterized by the fact whether or not the vector fields $A_{I}(x, v, \bar{v})_{M}$ can be globally extended to full $\mathrm{SU}(2)$ invariants or will be at most be invariant under a $\mathrm{U}(1)$ subgroup. 
Many of the $4 D$ features related to tensor gauge invariance and R-symmetry invariance remain relevant in $3 D$. Hence let us turn to the reduction of the Lagrangian (5.13) to three dimensions by using the expressions for the $4 D$ fields summarized in (5.7) and (5.3) and the Ricci scalar in (A.3). Starting from the first line in (5.13), we find

$$
\begin{aligned}
- & \frac{1}{2} E F_{I J} \mathcal{D}_{M} L_{i j}{ }^{I} \mathcal{D}^{M} L^{i j J}+E F_{I J} L_{i j}{ }^{I} L^{i j J}\left(\frac{1}{3} R+D\right) \\
= & \frac{1}{2} e F_{I J} \mathcal{D}_{\mu} L^{i}{ }_{j}{ }^{I} \mathcal{D}^{\mu} L^{j}{ }_{i}{ }^{J}-\frac{1}{2} e F_{I J} L^{i}{ }_{j}{ }^{I} L^{j}{ }_{i}{ }^{J}\left(\frac{1}{2} R+D-C^{2}\right) \\
& +\frac{1}{4} e F_{I J} \phi^{-2}\left(L^{i}{ }_{j}{ }^{I} Y^{0 j}{ }_{i}\right)\left(L^{k}{ }_{l}{ }^{J} Y^{0 l}{ }_{k}\right),
\end{aligned}
$$

where we used the homogeneity of $F_{I J}$ to express it in terms the $3 D$ scalars $L^{i}{ }_{j}$ defined in (5.7). ${ }^{3}$ We also suppressed a total derivative term (for this it is convenient to make use of the first equation (5.19)). Subsequently we consider the next few terms of (5.13) and reduce them to three dimensions,

$$
\begin{array}{rl}
E & F(L)_{I J}\left(E_{M}{ }^{I} E^{M J}-E^{M I} \mathcal{V}_{M}{ }^{i}{ }_{j} L_{i k}{ }^{J} \varepsilon^{j k}+G^{I} \bar{G}^{J}\right) \\
= & -\frac{1}{2} e F(L)_{I J}\left[\frac{1}{2} \phi^{-2}\left(L^{i}{ }_{j}{ }^{I} Y^{j}{ }_{i}^{0}\right)\left(L^{k}{ }^{J}{ }^{J} Y_{k}{ }_{k}{ }^{0}\right)+F(E)_{\mu \nu}{ }^{I} F(E)^{\mu \nu J}+Y^{p}{ }_{q}{ }^{I} Y^{q}{ }_{p}{ }^{J}\right] \\
& -\frac{1}{2} \mathrm{i} \varepsilon^{\mu \nu \rho} F(L)_{I J} F(E)_{\mu \nu}{ }^{I} L^{i}{ }_{j}{ }^{J} \mathcal{V}_{\rho}{ }^{j}{ }_{i} .
\end{array}
$$

Combining all these contributions with those coming form the last term in (5.13), we obtain the final result,

$$
\begin{aligned}
\left.\mathcal{L}_{\text {tensor }}\right|_{3 D}= & \frac{1}{2} e F(L)_{I J} \mathcal{D}_{\mu} L^{i}{ }_{j}{ }^{I} \mathcal{D}^{\mu} L^{j}{ }_{i}{ }^{J}-\frac{1}{2} e F(L)_{I J} L^{i}{ }_{j}{ }^{I} L^{j}{ }_{i}{ }^{J}\left(\frac{1}{2} R+D-C^{2}\right) \\
& -\frac{1}{2} e F(L)_{I J}\left[F(E)_{\mu \nu}{ }^{I} F(E)^{\mu \nu J}+Y^{p}{ }_{q}{ }^{I} Y^{q}{ }_{p}{ }^{J}\right] \\
& -\frac{1}{2} \mathrm{i} \varepsilon^{\mu \nu \rho} F(L)_{I J} F(E)_{\mu \nu}{ }^{I} L^{i}{ }_{j}{ }^{J} \mathcal{V}_{\rho}{ }^{j}{ }_{i} \\
& +\mathrm{i} \varepsilon^{\mu \nu \rho} F(L)_{I J K i}{ }^{j} \partial_{\mu} L^{i}{ }_{k}{ }^{I} \partial_{\nu} L^{k}{ }_{j}{ }^{J} E_{\rho}{ }^{K}
\end{aligned}
$$

where we note that the Kaluza-Klein vector multiplet again manifestly decouples. This should not come as a surprise as one can dualize the $3 D$ vector field $E_{\mu}$ to a scalar and then obtain a hypermultiplet Lagrangian, for which we have noted the same decoupling phenomenon. Let us stress that all the properties of the $4 D$ tensor Lagrangians related to the tensor potential $\chi_{\text {tensor }}(L)$ carry over to the three-dimensional context. However, the definition (5.18) in terms of the fields $L^{i}{ }_{j}$ acquires an explicit minus sign because $L^{i j I} L_{i j}{ }^{J}=-L^{i}{ }_{j}^{I} L^{j}{ }_{i}{ }^{J}$. As a result the equations (5.18) and (5.19) take the following form,

$$
\begin{aligned}
\chi_{\text {tensor }}(L) & =-2 F_{I J} L^{i}{ }_{j}{ }^{I} L_{i}^{j}{ }^{J}, \\
\frac{\partial \chi_{\text {tensor }}(L)}{\partial L^{i}{ }^{I}} & =-2 F_{I J}(L) L^{j}{ }_{i}{ }^{J}, \\
\frac{\partial^{2} \chi_{\text {tensor }}(L)}{\partial L^{i}{ }_{k} I \partial L^{k}{ }_{j} J} & =-2 F_{I J}(L) \delta_{i}{ }^{j} .
\end{aligned}
$$

\footnotetext{
${ }^{3}$ We remind the reader that the $L^{i}{ }_{j}$ are anti-hermitian.
} 


\subsubsection{The vector multiplet Lagrangian}

Finally, we turn to the bosonic Lagrangian for vector multiplets, whose evaluation is considerably more complicated, as the Kaluza-Klein vector multiplet will not decouple in this case. Therefore the number of vector multiplets will increase by one under the reduction. To avoid confusion with the discussion in subsection 5.2.2, we will use indices $\Lambda, \Sigma, \Xi, \ldots$ to label the $n+1$ off-shell $4 D$ vector multiplets. With the Kaluza-Klein vector multiplet we will thus obtain $n+23 D$ vector multiplets. As before we start from the bosonic terms of the $4 D$ Lagrangian, which take the form,

$$
\begin{aligned}
\left.\mathcal{L}_{\text {vector }}\right|_{4 D}= & E N_{\Lambda \Sigma}\left[X^{\Lambda} \bar{X}^{\Sigma}\left(\frac{1}{6} R-D\right)+\frac{1}{8} Y_{i j}{ }^{\Lambda} Y^{i j \Sigma}-\mathcal{D}_{M} X^{\Lambda} \mathcal{D}^{M} \bar{X}^{\Sigma}\right] \\
& -\frac{1}{8} E N_{\Lambda \Sigma} F_{M N}{ }^{\Lambda} F^{M N \Sigma}-\frac{1}{16} \mathrm{i} \varepsilon^{M N P Q} R_{\Lambda \Sigma} F_{M N}{ }^{\Lambda} F_{P Q}{ }^{\Sigma} \\
& +\frac{1}{8} E\left[\bar{X}^{\Lambda} N_{\Lambda \Sigma} F^{A B \Sigma} T_{A B}{ }^{i j} \varepsilon_{i j}-\frac{1}{8} \bar{X}^{\Lambda} N_{\Lambda \Sigma} \bar{X}^{\Sigma}\left(T_{A B}{ }^{i j} \varepsilon_{i j}\right)^{2}+\text { h.c. }\right],
\end{aligned}
$$

and is encoded in a holomorphic function $F(X)$ that is homogeneous of second degree. Its multiple derivatives are denoted by $F_{\Lambda \Sigma \Xi \ldots}$ and the second derivatives are decomposed into two real tensors, $N_{\Lambda \Sigma}=-\mathrm{i} F_{\Lambda \Sigma}+\mathrm{i} \bar{F}_{\Lambda \Sigma}$ and $R_{\Lambda \Sigma}=F_{\Lambda \Sigma}+\bar{F}_{\Lambda \Sigma}$, which we have used in the above expression.

As before, we reduce (5.29) in steps, starting with the first two terms,

$$
\begin{aligned}
E & N_{\Lambda \Sigma}\left[X^{\Lambda} \bar{X}^{\Sigma}\left(\frac{1}{6} R-D\right)+\frac{1}{8} Y_{i j}{ }^{\Lambda} Y^{i j \Sigma}\right] \\
= & \frac{1}{2} e \phi^{-1} N_{\Lambda \Sigma} X^{\Lambda} \bar{X}^{\Sigma}\left[\frac{1}{2} R-D+C^{2}+\frac{1}{4} \phi^{-2}\left(F(W)_{a b}{ }^{0}\right)^{2}+\phi^{-2}\left(\mathcal{D}_{\mu} \phi\right)^{2}\right] \\
& -\frac{1}{8} e \phi^{-1} N_{\Lambda \Sigma}\left[Y^{i}{ }_{j}{ }^{\Lambda} Y^{j}{ }_{i}^{\Sigma}+\phi^{-2} L^{p}{ }_{q}{ }^{\Lambda} L^{q}{ }_{p}{ }^{0} Y^{i}{ }_{j}{ }^{\Sigma} Y^{j}{ }_{i}{ }^{0}\right] \\
& +\frac{1}{4} e \phi^{-3} N_{\Lambda \Sigma}\left[X^{\Lambda} \bar{X}^{\Sigma}-\frac{1}{8} \phi^{-2} L^{p}{ }_{q}^{\Lambda} L^{q}{ }_{p}^{0} L^{r}{ }_{s}{ }^{\Sigma} L^{s}{ }_{r}{ }^{0}\right] Y^{i}{ }_{j}{ }^{0} Y^{j}{ }_{i}{ }^{0},
\end{aligned}
$$

where we made use of (5.3), (5.4) and (A.3), and we employed the identifications $\phi=L^{0}$ and $L^{p}{ }^{\Lambda} L^{q} p^{0}=-\frac{1}{2} x^{\Lambda} x^{0}-v^{\Lambda} \bar{v}^{0}-\bar{v}^{\Lambda} v^{0}$. The next terms related to the kinetic terms of $X^{\Lambda}$ and the various field-strengths reduce to

$$
\begin{aligned}
-E & N_{\Lambda \Sigma} \mathcal{D}_{M} X^{\Lambda} \mathcal{D}^{M} \bar{X}^{\Sigma}-\frac{1}{8} E N_{\Lambda \Sigma} F_{M N}{ }^{\Lambda} F^{M N \Sigma}-\frac{1}{16} \mathrm{i} \varepsilon^{M N P Q} R_{\Lambda \Sigma} F_{M N}{ }^{\Lambda} F_{P Q}{ }^{\Sigma} \\
= & -e \phi^{-1} N_{\Lambda \Sigma}\left[X^{\Lambda} \bar{X}^{\Sigma} C^{2}+\mathcal{D}_{\mu} X^{\Lambda} \mathcal{D}_{\mu} \bar{X}^{\Sigma}\right] \\
& +\frac{1}{4} \phi^{-2} N_{\Lambda \Sigma}\left(X^{\Lambda} \stackrel{\leftrightarrow}{\mathcal{D}}_{\mu} \bar{X}^{\Sigma}\right) \varepsilon^{\mu \nu \rho} F(W)_{\nu \rho}{ }^{0} \\
& -\frac{1}{8} e \phi^{-1} N_{\Lambda \Sigma}\left[F(W)_{\mu \nu}{ }^{\Lambda} F(W)^{\mu \nu \Sigma}+\phi^{-2} L^{p} q^{\Lambda} L^{q}{ }_{p}^{0} F(W)_{\mu \nu}{ }^{\Sigma} F(W)^{\mu \nu 0}\right] \\
& +\frac{1}{4} e \phi^{-3} N_{\Lambda \Sigma}\left[\frac{1}{2} X^{\Lambda} \bar{X}^{\Sigma}-\frac{1}{8} \phi^{-2} L_{q}^{p}{ }^{\Lambda} L^{q}{ }_{p}^{0} L^{r}{ }_{s}^{\Sigma} L_{r}^{s}{ }_{r}^{0}\right] F(W)_{\mu \nu}{ }^{0} F(W)^{\mu \nu 0} \\
& +\frac{1}{8} \mathrm{i} \varepsilon^{\mu \nu \rho} R_{\Lambda \Sigma}\left[F(W)_{\mu \nu}{ }^{\Lambda}+\frac{1}{2} \phi^{-2} L^{p} q^{\Lambda} L_{p}^{q}{ }^{0} F(W)_{\mu \nu}{ }^{0}\right] \partial_{\rho}\left(\phi^{-2} L^{r}{ }_{s}^{\Sigma} L_{r}^{s}{ }^{0}\right) \\
& -\frac{1}{16} e \phi N_{\Lambda \Sigma} \partial_{\mu}\left(\phi^{-2} L^{p}{ }_{q}^{\Lambda} L^{q}{ }_{p}^{0}\right) \partial^{\mu}\left(\phi^{-2} L^{r}{ }_{s}^{\Sigma} L_{r}^{s}{ }^{0}\right) .
\end{aligned}
$$


In the left hand side of this relation, $\mathcal{D}_{\mu} X^{\Lambda}$ denotes a $\mathrm{U}(1)$ covariant derivative with the connection $\mathcal{A}_{\mu}^{0}$ that was defined in (2.48),

$$
\mathcal{D}_{\mu} X^{\Lambda}=\left(\partial_{\mu}-b_{\mu}+\mathrm{i} \mathcal{A}_{\mu}^{0}\right) X^{\Lambda} .
$$

Finally we reduce the remaining terms

$$
\begin{aligned}
\frac{1}{8} E & {\left[\bar{X}^{\Lambda} N_{\Lambda \Sigma} F^{A B \Sigma} T_{A B}{ }^{i j} \varepsilon_{i j}-\frac{1}{8} \bar{X}^{\Lambda} N_{\Lambda \Sigma} \bar{X}^{\Sigma}\left(T_{A B}{ }^{i j} \varepsilon_{i j}\right)^{2}\right]+\text { h.c. } } \\
= & \frac{1}{8} \mathrm{i} e \phi^{-3} N_{\Lambda \Sigma} \bar{X}^{\Lambda}\left[\left(\bar{v}^{0} \overleftrightarrow{\mathcal{D}}_{\mu} x^{0}\right)-\frac{\bar{v}^{0}}{L^{0}+\frac{1}{2} x^{0}}\left(v^{0} \overleftrightarrow{\mathcal{D}}_{\mu} \bar{v}^{0}\right)\right] \\
& \times\left[\phi \partial^{\mu}\left(\phi^{-2} L^{p} q^{\Sigma} L^{q}{ }_{p}^{0}\right)+e^{-1} \varepsilon^{\mu \nu \rho}\left(F(W)_{\nu \rho}{ }^{\Sigma}+\frac{1}{2} \phi^{-2} L^{p} q^{\Sigma} L^{q}{ }_{p}^{0} F(W)_{\nu \rho}{ }^{0}\right)\right] \\
& +\frac{1}{16} e \phi^{-5} N_{\Lambda \Sigma} \bar{X}^{\Lambda} \bar{X}^{\Sigma}\left[\left(\bar{v}^{0} \overleftrightarrow{\mathcal{D}}_{a} x^{0}\right)-\frac{\bar{v}^{0}}{L^{0}+\frac{1}{2} x^{0}}\left(v^{0} \stackrel{\leftrightarrow}{\mathcal{D}}_{a} \bar{v}^{0}\right)\right]^{2}+\text { h.c. }
\end{aligned}
$$

Because the number of vector multiplets is increased by the presence of the KaluzaKlein vector multiplet, we extend the range of the indices $\{\Lambda\}$ to $\{A\}=\{0, \Lambda\}$, where the index $A=0$ refers to the Kaluza-Klein vector multiplet. Up to terms that involve derivatives of the scalar fields and the epsilon tensor, the $3 D$ Lagrangian can then be written as

$$
\begin{aligned}
\left.\mathcal{L}_{\text {vector }}\right|_{3 D}= & -\frac{1}{2} e \mathcal{F}_{A B}(L) L_{q}^{p}{ }^{A} L^{q}{ }_{p}{ }^{B}\left[\frac{1}{2} R-D-C^{2}\right] \\
& -\frac{1}{2} e \mathcal{F}_{A B}(L)\left[F(W)_{\mu \nu}{ }^{A} F(W)^{\mu \nu B}+Y^{i}{ }_{j}{ }^{A} Y^{j}{ }_{i}{ }^{B}\right] .
\end{aligned}
$$

Here we have simply collected all the corresponding terms of (5.30) and (5.31), which lead to the following expressions for the tensor $\mathcal{F}_{A B}(L)$,

$$
\begin{aligned}
\mathcal{F}_{\Lambda \Sigma} & =\frac{1}{4 L^{0}} N_{\Lambda \Sigma} \\
\mathcal{F}_{\Lambda 0} & =\mathcal{F}_{0 \Lambda}=\frac{1}{8\left(L^{0}\right)^{3}} N_{\Lambda \Sigma} L^{p}{ }^{{ }^{\Sigma}} L^{q}{ }_{p}{ }^{0} \\
\mathcal{F}_{00} & =\frac{1}{16\left(L^{0}\right)^{3}} N_{\Lambda \Sigma}\left[L^{p}{ }_{q}^{\Lambda} L^{q}{ }^{\Sigma}{ }^{\Sigma}+\frac{3 L^{p} q^{\Lambda} L^{q} p^{0} L^{r}{ }_{s}{ }^{\Sigma} L_{r}^{s}{ }^{0}}{2\left(L^{0}\right)^{2}}\right] .
\end{aligned}
$$

Furthermore, one easily verifies that the direct analogue of the tensor potential that was introduced earlier in $(5.18),{ }^{4}$

$$
\begin{aligned}
\chi_{\text {vector }} & \equiv-2 \mathcal{F}_{A B} L^{p}{ }_{q}^{A} L^{q}{ }_{p}^{B} \\
& =-\frac{N_{\Lambda \Sigma}}{4 L^{0}}\left[L^{p}{ }_{q}^{\Lambda} L^{q}{ }_{p}{ }^{\Sigma}+\frac{L^{p}{ }^{\Lambda} L^{q}{ }_{p}^{0} L^{r}{ }_{s}{ }^{\Sigma} L_{r}^{s}{ }^{0}}{2\left(L^{0}\right)^{2}}\right] \\
& =\frac{2 N_{\Lambda \Sigma} X^{\Lambda} \bar{X}^{\Sigma}}{L^{0}},
\end{aligned}
$$

\footnotetext{
${ }^{4}$ Note that a minus sign has to be introduced in the definition below because the quadratic form $L^{p}{ }_{q} L_{q}$ that we are using here is non-positive!
} 
is a homogeneous function of first degree and is manifestly invariant under the symmetries (5.6). To prove the identity between the second and third line one may use the following convenient expression for $X^{\Lambda}$ (cf. (5.8)),

$$
X^{\Lambda}=\frac{1}{2} \mathrm{i} \bar{v}^{\Lambda}-\frac{1}{4} \mathrm{i}\left[x^{\Lambda}-\frac{L^{p} q^{\Lambda} L^{q} p^{0}}{L^{0}}\right] \frac{\bar{v}^{0}}{L^{0}+\frac{1}{2} x^{0}} .
$$

The reader may verify that the application of a $3 D \mathrm{SU}(2)$ transformation on the righthand side of (5.37) takes the form of an U(1) transformation on the left-hand side with parameter $\Lambda_{A}$ defined in (2.45). Therefore U(1) invariant products such as $X^{\Lambda} \bar{X}^{\Sigma}$ should take an $\mathrm{SU}(2)$ invariant form. In particular we find

$$
-8 X^{(\Lambda} \bar{X}^{\Sigma)}=L_{q}^{p}{ }^{\Lambda} L_{p}^{q}{ }^{\Sigma}+\frac{L^{p}{ }^{\Lambda} L^{q}{ }_{p}^{0} L_{s}^{r}{ }^{\Sigma} L_{r}^{s}{ }^{0}}{2\left(L^{0}\right)^{2}}
$$

which indeed confirms the last identity in (5.36). Likewise $F_{\Lambda \Sigma}(X)$ is also $\mathrm{U}(1)$ invariant, and must therefore be $\mathrm{SU}(2)$ invariant as well. From this observation it follows directly that $N_{\Lambda \Sigma}=-\mathrm{i}\left(F_{\Lambda \Sigma}-\bar{F}_{\Lambda \Sigma}\right)$, the functions $\mathcal{F}_{A B}$ and $\chi_{\text {vector }}$ are all SU(2) invariant as well.

We should point out that all properties derived above are so far consistent with the fact that there exists a reflection associated with (3.3) that correspondingly interchanges the vector and the tensor multiplets. This explains the different sign of the terms proportional to the field $D$ in the two Lagrangians (5.27) and (5.34). We will see that this relation between tensor and vector supermultiplets is also valid for the remaining terms in the full Lagrangians. Therefore, the remainder of this section will be devoted to a detailed derivation of the bosonic terms of the vector Lagrangian in order to isolate the intricate features that are crucial for establishing the relationship with the tensor Lagrangian.

Before specifying the remaining terms in the Lagrangian (5.34), we present a convenient expression based on the derivatives of $X^{\Lambda}$ with respect to the components of $L^{p}{ }^{A}$,

$$
\begin{aligned}
\delta X^{\Lambda}= & \frac{v^{0} \delta \bar{v}^{0}-\bar{v}^{0} \delta v^{0}}{2 L^{0}\left(L^{0}+\frac{1}{2} x^{0}\right)} X^{\Lambda} \\
& -\frac{\mathrm{i} \bar{v}^{0}}{4 L^{0}}\left[\left(\delta x^{\Lambda}+\delta x^{0} \frac{L^{p} q^{0} L^{q} p^{\Lambda}}{2\left(L^{0}\right)^{2}}\right)+\frac{\bar{v}^{0}}{L^{0}+\frac{1}{2} x^{0}}\left(\delta v^{\Lambda}+\delta v^{0} \frac{L^{p} q^{0} L^{q} p^{\Lambda}}{2\left(L^{0}\right)^{2}}\right)\right. \\
& \left.-\frac{v^{0}}{L^{0}-\frac{1}{2} x^{0}}\left(\delta \bar{v}^{\Lambda}+\delta \bar{v}^{0} \frac{L^{p} q^{0} L^{q} p^{\Lambda}}{2\left(L^{0}\right)^{2}}\right)\right]
\end{aligned}
$$

Using that $F_{\Lambda \Sigma}(X)$ is the second derivative of a holomorphic homogeneous function of degree two, we derive the following two identities,

$$
\frac{\partial F_{\Lambda \Sigma}(X)}{\partial L^{p} q^{\Xi}}=\frac{\partial F_{\Lambda \Xi}(X)}{\partial L^{p} q^{\Sigma}}, \quad \frac{\partial F_{\Lambda \Sigma}(X)}{\partial L^{p} q^{0}}=\frac{\partial F_{\Lambda \Sigma}(X)}{\partial L^{p} q^{\Xi}} \frac{L_{s}^{r}{ }^{0} L_{r}^{s} \Xi}{2\left(L^{0}\right)^{2}},
$$

where the second equation follows directly from (5.39). Furthermore we note the identities

$$
\begin{aligned}
& \frac{\partial F_{\Lambda \Sigma}(X)}{\partial L^{r}{ }^{\Xi}} L_{q}^{r}=-L_{r}^{p 0} \frac{\partial F_{\Lambda \Sigma}(X)}{\partial L^{q} r^{\Xi}}=\mathrm{i} L^{0} \frac{\partial F_{\Lambda \Sigma}(X)}{\partial L^{q} p^{\Xi}}, \\
& \frac{\partial \bar{F}_{\Lambda \Sigma}(\bar{X})}{\partial L^{r} p^{\Xi}} L_{q}^{r}=-L_{r}^{p^{0}} \frac{\partial \bar{F}_{\Lambda \Sigma}(\bar{X})}{\partial L^{q}{ }_{r} \Xi}=-\mathrm{i} L^{0} \frac{\partial \bar{F}_{\Lambda \Sigma}(\bar{X})}{\partial L^{q} p^{\Xi}} .
\end{aligned}
$$


Subsequently we make use of the fact that $F_{\Lambda \Sigma}(X)$ is a homogeneous function of zeroth degree, so that it is invariant under complex scale transformations of the $4 D$ fields $X^{\Xi}$. When regarding $F_{\Lambda \Sigma}(X)$ as function of the $3 D$ fields, it must be an $\mathrm{SU}(2)$ invariant homogeneous function of zeroth degree. Moreover, close inspection based on (5.37) shows that it must be a homogeneous function of the $L^{p}{ }_{q}^{\Xi}$ and $L^{p}{ }^{0}$ separately. Exploiting the second equation (5.40), we thus derive the following results based on homogeneity and $\mathrm{SU}(2)$ invariance,

$$
\begin{aligned}
& \frac{\partial F_{\Lambda \Sigma}}{\partial L^{p}{ }_{q} \Xi} L_{q}^{p}=0, \quad \frac{\partial F_{\Lambda \Sigma}}{\partial L^{p}{ }_{q} \Xi} L_{q}^{p} \Xi=0, \quad \frac{\partial F_{\Lambda \Sigma}}{\partial L^{p}{ }^{0}} L_{q}^{p}{ }^{0}=0, \\
& \frac{\partial F_{\Lambda \Sigma}}{\partial L^{q}{ }_{r} \Xi}\left(L^{p}{ }_{r}^{\Xi}+L^{p}{ }_{r}{ }^{0} \frac{L^{s}{ }^{0} L_{s}^{t} \Xi}{2\left(L^{0}\right)^{2}}\right)=0,
\end{aligned}
$$

where the first equation, while consistent with homogeneity, is actually derived from (5.39). Furthermore the homogeneity of $F(X)$ implies that $\delta F_{\Lambda \Sigma}$ under any variations $\delta X^{\Xi}$ must satisfy $\delta F_{\Lambda \Sigma} X^{\Lambda}=0$, so that

$$
\frac{\partial F_{\Lambda \Sigma}}{\partial L^{A t} u}\left[L^{p}{ }_{q}^{\Lambda} L_{p}^{q} p^{\Sigma}+\frac{L^{p} q^{\Lambda} L^{q} p^{0} L^{r}{ }_{s}^{\Sigma} L_{r}^{s}{ }^{0}}{2\left(L^{0}\right)^{2}}\right]=0
$$

where we again made use of (5.38).

The above results can straightforwardly be used to derive a number of specific results that confirm the relation with the tensor multiplet Lagrangians. First of all, we may verify by using (5.40) and (5.43) that the derivative ${ }^{5}$ of $\mathcal{F}_{A B}$ in (5.35) with respect to $L^{p}{ }_{q}^{C}$, denoted by $\mathcal{F}_{A B C^{q}} p$, satisfies

$$
\mathcal{F}_{A B C}^{p}{ }_{q}=\mathcal{F}_{(A B C)}^{p}{ }^{p},
$$

which corresponds to the first equation given in (5.14) in the context of the tensor multiplets. Then we have already argued that the $F_{A B}(L)$ must be $\mathrm{SU}(2)$ invariant; moreover they are manifestly homogeneous functions of degree -1 in terms of the $3 D$ fields $F^{p}{ }^{A}$. Therefore we derive the identity

$$
\mathcal{F}_{A B C}{ }^{q}{ }_{r} L_{p}^{r}{ }^{C}=-\frac{1}{2} \delta^{p}{ }_{q} \mathcal{F}_{A B}
$$

which is precisely analogous to (5.17), considered in the context of the tensor Lagrangian. Furthermore, from (5.43) one can verify the following relations,

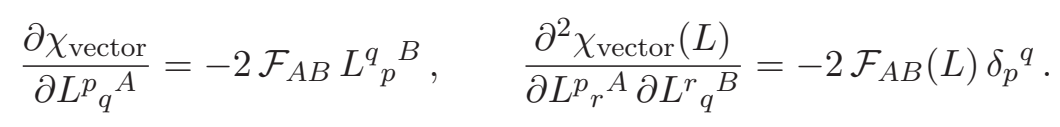

\footnotetext{
${ }^{5}$ Observe that with the definitions of this paper we have

$$
\frac{\partial L_{q}^{p}}{\partial L^{r}}=\delta_{r}^{p} \delta_{q}^{t}, \quad \frac{\partial L^{0}}{\partial L_{q}^{p}}=-\frac{L_{p}^{q}{ }^{0}}{2 L^{0}} .
$$
}


which are analogous to the equations (5.28) derived for tensor multiplets. For future use we give the explicit expressions for the independent components of $\mathcal{F}_{A B C}{ }^{p}$,

$$
\begin{aligned}
& \mathcal{F}_{\Lambda \Sigma \Xi^{p}}^{p}=\frac{1}{4 L^{0}} \frac{\partial N_{\Lambda \Sigma}}{\partial L^{q}{ }^{\Xi}}, \\
& \mathcal{F}_{\Lambda \Sigma 0}{ }^{p}{ }_{q}=\frac{1}{8\left(L^{0}\right)^{3}} N_{\Lambda \Sigma} L_{q}^{p}+\frac{1}{8\left(L^{0}\right)^{3}} \frac{\partial N_{\Lambda \Sigma}}{\partial L^{q} p^{\Xi}} L_{s}^{r} L_{r}^{s}{ }_{r}^{0}, \\
& \mathcal{F}_{\Lambda 00}^{p}{ }_{q}=\frac{1}{8\left(L^{0}\right)^{3}} N_{\Lambda \Sigma}\left[L_{q}^{p \Sigma}+\frac{3 L_{s}^{r \Sigma} L_{r}^{s}{ }_{r}^{0}}{2\left(L^{0}\right)^{2}} L_{q}^{p 0}\right] \\
& -\frac{1}{8\left(L^{0}\right)^{3}} \frac{\partial N_{\Sigma \Xi}}{\partial L^{q} p^{\Lambda}} L_{s}^{r \Sigma} L_{r}^{s} \Xi \\
& \mathcal{F}_{000}{ }^{p}{ }_{q}=\frac{3}{32\left(L^{0}\right)^{5}} N_{\Lambda \Sigma}\left[L^{r}{ }_{s}^{\Lambda} L_{r}^{s}{ }_{r}^{\Sigma} L^{p}{ }_{q}^{0}+2 L_{s}^{r \Lambda} L_{r}^{s}{ }_{r}^{0} L_{q}^{p}+\frac{5 L_{s}^{r \Lambda} L_{r}^{s}{ }_{r}^{0} L_{u}^{t}{ }^{\Sigma} L_{t}^{u 0}}{2\left(L^{0}\right)^{2}} L_{q}^{p}{ }_{q}^{0}\right] \\
& -\frac{1}{16\left(L^{0}\right)^{5}} \frac{\partial N_{\Lambda \Sigma}}{\partial L_{p}^{q} \Xi} L_{s}^{r} L_{r}^{s} L_{u}^{t} L_{u}^{\Xi} L_{t}^{u 0},
\end{aligned}
$$

where we made use of the relations (5.40) and (5.43). To verify their correctness one can, for instance, verify the validity of (5.45).

To continue we will also need the following result for the covariant derivatives $\mathcal{D}_{\mu} X^{\Lambda}$ in terms of the three-dimensional fields,

$$
\begin{aligned}
\mathcal{D}_{\mu} X^{\Lambda}= & \frac{1}{2} \mathrm{i} \mathcal{D}_{\mu} \bar{v}^{\Lambda}-\frac{1}{4} \mathrm{i}\left[\mathcal{D}_{\mu} x^{\Lambda}-\frac{\mathcal{D}_{\mu} L^{p} q^{\Lambda} L^{q} p^{0}}{L^{0}}\right] \frac{\bar{v}^{0}}{L^{0}+\frac{1}{2} x^{0}} \\
& -\mathrm{i} \frac{L^{p}{ }^{\Lambda} L^{q} p^{0}}{8\left(L^{0}\right)^{3}}\left[\left(\bar{v}^{0} \stackrel{\leftrightarrow}{\mathcal{D}}_{\mu} x^{0}\right)-\frac{\bar{v}^{0}}{L^{0}+\frac{1}{2} x^{0}}\left(v^{0} \stackrel{\leftrightarrow}{\mathcal{D}}_{\mu} \bar{v}^{0}\right)\right],
\end{aligned}
$$

which has been derived by making use again of (5.39). One then proceeds to evaluate the remaining terms of the action which all involve derivatives of the scalar fields. First let us collect all the terms quadratic in these derivatives from (5.30), (5.31) and (5.33),

$$
\begin{aligned}
e N_{\Lambda \Sigma}[ & \frac{1}{2} \phi^{-3} X^{\Lambda} \bar{X}^{\Sigma}\left(\mathcal{D}_{\mu} \phi\right)^{2}-\phi^{-1} \mathcal{D}_{\mu} X^{\Lambda} \mathcal{D}^{\mu} \bar{X}^{\Sigma} \\
& +\frac{1}{16} \phi^{-5} \bar{X}^{\Lambda} \bar{X}^{\Sigma}\left[\left(\bar{v}^{0} \overleftrightarrow{\mathcal{D}}_{\mu} x^{0}\right)-\frac{\bar{v}^{0}}{L^{0}+\frac{1}{2} x^{0}}\left(v^{0} \overleftrightarrow{\mathcal{D}}_{\mu} \bar{v}^{0}\right)\right]^{2}+\text { h.c. } \\
& +\frac{1}{8} \mathrm{i} \phi^{-2} \bar{X}^{\Lambda}\left[\left(\bar{v}^{0} \stackrel{\leftrightarrow}{\mathcal{D}}_{\mu} x^{0}\right)-\frac{\bar{v}^{0}}{L^{0}+\frac{1}{2} x^{0}}\left(v^{0} \stackrel{\leftrightarrow}{\mathcal{D}}_{\mu} \bar{v}^{0}\right)\right] \partial^{\mu}\left(\phi^{-2} L^{p}{ }^{\Sigma} L_{p}^{q}{ }_{p}^{0}\right)+\text { h.c. } \\
& \left.-\frac{1}{16} \phi \partial_{\mu}\left(\phi^{-2} L_{q}^{p}{ }^{\Lambda} L^{q}{ }_{p}^{0}\right) \partial^{\mu}\left(\phi^{-2} L_{s}^{r}{ }_{s} L_{r}^{s}{ }_{r}^{0}\right)\right]
\end{aligned}
$$


To write this expression in terms of the $3 D$ fields we first derive the following three identities,

$$
\begin{aligned}
& \mathrm{i} X^{\Lambda}\left[\left(v^{0} \stackrel{\leftrightarrow}{\mathcal{D}}_{\mu} x^{0}\right)-\frac{v^{0}}{L^{0}+\frac{1}{2} x^{0}}\left(\bar{v}^{0} \stackrel{\leftrightarrow}{\mathcal{D}}_{\mu} v^{0}\right)\right]+\text { h.c. } \\
& =-L^{0} L^{p}{ }_{q}^{\Lambda} \mathcal{D}_{\mu} L^{q}{ }_{p}{ }^{0}-\frac{L^{r}{ }^{\Lambda}{ }^{\Lambda} L_{r}{ }^{0}}{2 L^{0}} L^{p}{ }_{q}{ }^{0} \mathcal{D}_{\mu} L^{q}{ }_{p}{ }^{0}, \\
& \mathcal{D}_{\mu} X^{(\Lambda} \mathcal{D}^{\mu} \bar{X}^{\Sigma)} \\
& =-\frac{1}{8} \mathcal{D}_{\mu} L^{p}{ }_{q}^{\Lambda} \mathcal{D}^{\mu} L^{q}{ }_{p}{ }^{\Sigma}-\frac{1}{16\left(L^{0}\right)^{2}} L^{p}{ }_{q}^{0} \mathcal{D}_{\mu} L^{q}{ }_{p}{ }^{\Lambda} L^{r}{ }_{s}{ }^{0} \mathcal{D}^{\mu} L_{r}^{s}{ }^{\Sigma} \\
& -\frac{L^{r}{ }_{s}{ }^{0} L^{s}{ }_{r}(\Lambda}{8\left(L^{0}\right)^{2}} \mathcal{D}_{\mu} L^{p}{ }^{\Sigma}{ }^{\Sigma)} \mathcal{D}^{\mu} L^{q}{ }_{p}{ }^{0}-\frac{L^{r}{ }_{s}{ }^{0} L_{r}^{s}{ }_{r}(\Lambda}{16\left(L^{0}\right)^{4}} \mathcal{D}_{\mu} L^{p}{ }^{p}{ }^{\Sigma)} L^{q}{ }_{p}{ }^{0} \mathcal{D}^{\mu} L^{t}{ }_{u}{ }^{0} L^{u}{ }_{t}{ }^{0} \\
& +\frac{1}{64\left(L^{0}\right)^{6}} L^{p}{ }^{\Lambda} L_{p}^{q}{ }^{0} L^{r}{ }_{s}^{\Sigma} L_{r}^{s}{ }_{r}^{0}\left|\left(\bar{v}^{0} \stackrel{\leftrightarrow}{\mathcal{D}}_{\mu} x^{0}\right)-\frac{\bar{v}^{0}}{L^{0}+\frac{1}{2} x^{0}}\left(v^{0} \stackrel{\leftrightarrow}{\mathcal{D}}_{\mu} \bar{v}^{0}\right)\right|^{2}, \\
& \left|\left(\bar{v}^{0} \stackrel{\leftrightarrow}{\mathcal{D}}_{\mu} x^{0}\right)-\frac{\bar{v}^{0}}{L^{0}+\frac{1}{2} x^{0}}\left(v^{0} \overleftrightarrow{\mathcal{D}}_{\mu} \bar{v}^{0}\right)\right|^{2} \\
& =-2\left(L^{0}\right)^{2}\left(\mathcal{D}_{\mu} L^{p}{ }_{q}^{0} \mathcal{D}^{\mu} L^{q}{ }_{p}{ }^{0}\right)-\left(L^{p}{ }_{q}^{0} \mathcal{D}_{\mu} L^{q}{ }_{p}{ }^{0}\right)^{2} .
\end{aligned}
$$

The right-hand side of these expressions is manifestly invariant under the emergent $3 D$ $\mathrm{SU}(2)$ R-symmetry, as is to be expected because the expressions on the left-hand side are invariant under the the $4 D \mathrm{U}(1)$ R-symmetry. Collecting the various terms one can verify that all the terms quadratic in the derivatives of the scalar fields combine into the following form,

$$
\left.\mathcal{L}_{\text {vector }}\right|_{3 D}=\frac{1}{2} e \mathcal{F}_{A B}(L) \mathcal{D}_{\mu} L^{p}{ }^{A} \mathcal{D}^{\mu} L^{q}{ }^{B} .
$$

What remains to evaluate are the terms linear in the field strengths. Collecting those terms gives rise to

$$
\begin{aligned}
\mathrm{i} \varepsilon^{\mu \nu \rho} N_{\Lambda \Sigma}[ & -\frac{1}{4} \mathrm{i} \phi^{-2}\left(X^{\Lambda} \stackrel{\leftrightarrow}{\mathcal{D}}_{\mu} \bar{X}^{\Sigma}\right) F(W)_{\nu \rho}{ }^{0} \\
& +\frac{1}{8} \phi^{-3}\left(\bar{X}^{\Lambda}\left[\left(\bar{v}^{0} \stackrel{\leftrightarrow}{\mathcal{D}}_{\mu} x^{0}\right)-\frac{\bar{v}^{0}}{L^{0}+\frac{1}{2} x^{0}}\left(v^{0} \stackrel{\leftrightarrow}{\mathcal{D}}_{\mu} \bar{v}^{0}\right)\right]+\text { h.c }\right) \\
& \left.\times\left(F(W)_{\nu \rho}{ }^{\Sigma}+\frac{1}{2} \phi^{-2} L^{p}{ }^{\Sigma}{ }^{\Sigma} L^{q}{ }_{p}^{0} F(W)_{\nu \rho}{ }^{0}\right)\right] \\
+\frac{1}{8} \mathrm{i} \varepsilon^{\mu \nu \rho} R_{\Lambda \Sigma} & {\left[F(W)_{\mu \nu}{ }^{\Lambda}+\frac{1}{2} \phi^{-2} L^{p}{ }_{q}^{\Lambda} L_{p}^{q}{ }_{p}^{0} F(W)_{\mu \nu}{ }^{0}\right] \partial_{\rho}\left(\phi^{-2} L^{r}{ }_{s}{ }^{\Sigma} L_{r}^{s}{ }_{r}{ }^{0}\right) }
\end{aligned}
$$

These terms can be rewritten by using identities similar to the ones given in (5.50), which lead to the following expression,

$$
\begin{aligned}
& \mathrm{i} \varepsilon^{\mu \nu \rho} N_{\Lambda \Sigma}\left[\frac{1}{16\left(L^{0}\right)^{3}}\left(L^{p}{ }^{0}{ }^{0} L_{r}^{q}{ }^{\Lambda} \mathcal{D}_{\mu} L^{r}{ }^{\Sigma}{ }^{\Sigma}-\frac{3 L^{s} t^{\Lambda} L^{t}{ }_{s}{ }^{0}}{2\left(L^{0}\right)^{2}} L^{p}{ }^{\Sigma}{ }^{\Sigma} L^{q}{ }_{r}{ }^{0} \mathcal{D}_{\mu} L^{r}{ }_{p}{ }^{0}\right) F(W)_{\nu \rho}{ }^{0}\right. \\
& \left.-\frac{1}{8\left(L^{0}\right)^{3}} L^{p}{ }^{\Lambda} L^{q}{ }_{r}^{0} \mathcal{D}_{\mu} L^{r}{ }_{p}^{0} F(W)_{\nu \rho}{ }^{\Sigma}\right] \\
& +\frac{1}{8} \mathrm{i} \varepsilon^{\mu \nu \rho} R_{\Lambda \Sigma} \partial_{\mu}\left(\frac{L^{p} q^{\Lambda} L^{q} p^{0}}{\left(L^{0}\right)^{2}}\right)\left[F(W)_{\nu \rho}{ }^{\Sigma}+\frac{L_{t}^{s}{ }^{\Sigma} L_{s}^{t}{ }^{0}}{2\left(L^{0}\right)^{2}} F(W)_{\nu \rho}{ }^{0}\right] \text {. }
\end{aligned}
$$


This expression is manifestly invariant under local $\mathrm{SU}(2)$ transformations as well as under gauge transformations of the fields $W_{\mu}{ }^{A}$. This is in contrast with the situation encountered for generic tensor multiplets discussed in subsection 5.2.2, where we argued that this is not the case in general (see, in particular the discussion related to the equations (5.21)-(5.24)). Hence we conclude that the models obtained by dimensional reduction from $4 D$ vector multiplets belong to a restricted class. As we shall discuss in the next subsection 5.3, this implies that certain tensor multiplet models are not in the image of the c-map. This does not come as a surprise as such a phenomenon has been noted earlier for hypermultiplets [1].

It remains to verify explicitly that (5.53) has the same structure as the two last terms in (5.27). Let us therefore first extract the terms proportional to the $\mathrm{SU}(2)$ connections $\mathcal{A}_{\mu}^{p}{ }^{p}$. We note that the covariant derivatives in (5.53) appear in the form $\operatorname{tr}\left[L_{1} L_{2} \mathcal{D}_{\mu} L_{3}\right]$, so that the terms proportional to the gauge connection $\mathcal{A}_{\mu}$ take the form

$$
\frac{1}{2} \operatorname{tr}\left[L_{1} L_{2}\left[\mathcal{A}, L_{3}\right]\right]=\frac{1}{2} \operatorname{tr}\left[L_{1} L_{3}\right] \operatorname{tr}\left[L_{2} \mathcal{A}_{\mu}\right]-\frac{1}{2} \operatorname{tr}\left[L_{2} L_{3}\right] \operatorname{tr}\left[L_{1} \mathcal{A}_{\mu}\right],
$$

where we have used that $L_{1}, L_{2}, L_{3}$ and $\mathcal{A}_{\mu}$ are traceless, anti-hermitian two-by-two matrices. Collecting the various terms from (5.53) linear in the connection is now straightforward and leads to

$$
\left.\mathcal{L}\right|_{3 D}=-\frac{1}{2} \mathrm{i} \varepsilon^{\mu \nu \rho} \mathcal{F}_{A B}(L) F(W)_{\mu \nu}{ }^{A} L_{q}^{p}{ }^{B} \mathcal{A}_{\rho}{ }^{q} p
$$

This term takes exactly the same form as the corresponding term in the Lagrangian (5.27).

Finally we have to show that the terms in (5.53) with an ordinary derivative are equal to

$$
\left.\mathcal{L}\right|_{3 D}=\mathrm{i} \varepsilon^{\mu \nu \rho} \mathcal{F}_{A B C^{p}}{ }_{q} \partial_{\mu} L^{q}{ }_{r}^{A} \partial_{\nu} L_{p}^{r}{ }^{B} W_{\rho}^{C}
$$

upon adding a total derivative. In this way the terms in (5.53) that involve $R_{\Lambda \Sigma}$ can be written such that they become proportional to $\partial_{\mu} R_{\Lambda \Sigma}$ times a bare gauge field. Making use of (5.41) one then derives the following identity,

$$
\begin{aligned}
& \partial_{[\mu} R_{\Lambda \Sigma} \partial_{\nu]}\left(\frac{L^{p} q^{\Lambda} L^{q} p^{0}}{\left(L^{0}\right)^{2}}\right) \\
& =\frac{1}{\left(L^{0}\right)^{2}} \frac{\partial R_{\Lambda \Sigma}}{\partial L^{p} \Xi}\left[\partial_{[\mu} L_{q}^{p}{ }_{q}^{\Lambda} \partial_{\nu]} L_{s}^{r \Xi} L_{r}^{s 0}+\partial_{[\mu} L_{q}^{p \Lambda} \partial_{\nu]} L_{s}^{r 0} L_{r}^{s} \Xi\right. \\
& +\frac{L_{u}^{t}{ }_{u}^{\Lambda} L_{t}^{u} t^{0}}{2\left(L^{0}\right)^{2}}\left(\partial_{[\mu} L_{q}^{p 0} \partial_{\nu]} L_{s}^{r \Xi} L_{r}^{s}{ }_{r}^{0}+\partial_{[\mu} L_{q}^{p 0} \partial_{\nu]} L_{s}^{r 0} L_{r}^{s} \Xi+2 \partial_{[\mu} L_{q}^{p \Xi} \partial_{\nu]} L_{s}^{r 0} L_{r}^{s}{ }_{r}^{0}\right) \\
& \left.+\frac{L^{t}{ }_{u}^{\Lambda} L^{u}{ }^{0}}{2\left(L^{0}\right)^{2}} \frac{L^{v}{ }_{w}{ }^{\Xi} L^{w}{ }_{v}^{0}}{\left(L^{0}\right)^{2}} \partial_{[\mu} L_{q}^{p 0} \partial_{\nu]} L_{s}^{r}{ }^{0} L_{r}^{s}{ }_{r}^{0}\right] .
\end{aligned}
$$

Since $\mathcal{F}_{A B C}{ }^{p}$ is defined in terms of $N_{\Lambda \Sigma}$ and its derivatives, we have to convert these terms so that the result is either proportional to $N_{\Lambda \Sigma}$ or to its derivative. This can be achieved by making use of (5.41), from which one derives

$$
\frac{\partial R_{\Lambda \Sigma}}{\partial L_{p}^{q} \Xi}=\frac{1}{L^{0}} \frac{\partial N_{\Lambda \Sigma}(X)}{\partial L_{p}^{r} \Xi} L_{q}^{r}=-\frac{1}{L^{0}} L_{r}^{p} \frac{\partial N_{\Lambda \Sigma}}{\partial L_{r}^{q} \Xi} .
$$

With the above results, upon using (5.40), (5.42), and

$$
L_{q}^{p}{ }_{q}^{[A} L_{s}^{r}{ }^{B]}=-\frac{1}{2} \delta^{p}{ }_{s} L^{r}{ }_{t}^{[A} L^{t}{ }_{q}^{B]}+\frac{1}{2} \delta^{r}{ }_{q} L^{p}{ }_{t}\left[A L^{t}{ }_{s}^{B]},\right.
$$


to rearrange the various contractions of $\mathrm{SU}(2)$ indices, one can verify that all terms lead indeed to (5.56).

Combining the various results derived in this subsection, the resulting $3 D$ vector multiplet Lagrangian reads as follows,

$$
\begin{aligned}
\left.\mathcal{L}_{\text {vector }}\right|_{3 D}= & \frac{1}{2} e \mathcal{F}(L)_{A B} \mathcal{D}_{\mu} L^{p}{ }_{q}^{A} \mathcal{D}^{\mu} L^{q}{ }_{p}^{B}-\frac{1}{2} e \mathcal{F}(L)_{A B} L^{p}{ }_{q}^{A} L^{q}{ }_{p}^{B}\left(\frac{1}{2} R-D-C^{2}\right) \\
& -\frac{1}{2} e \mathcal{F}(L)_{A B}\left[F(W)_{\mu \nu}{ }^{A} F(W)^{\mu \nu B}+Y^{i}{ }_{j}{ }^{A} Y^{j}{ }_{i}{ }^{B}\right] \\
& -\frac{1}{2} \mathrm{i} \varepsilon^{\mu \nu \rho} \mathcal{F}(L)_{A B} F(W)_{\mu \nu}{ }^{A} L^{p}{ }_{q}^{B} \mathcal{A}_{\rho}{ }^{q} p \\
& +\mathrm{i} \varepsilon^{\mu \nu \rho} \mathcal{F}(L)_{A B C}{ }_{q}{ }_{q} \partial_{\mu} L^{q}{ }_{r}^{A} \partial_{\nu} L^{r}{ }_{p}^{B} W_{\rho}^{C},
\end{aligned}
$$

which coincides with that of the tensor Lagrangian (5.27), except that the $\mathrm{SU}(2)$ indices $i, j, \ldots$ have been interchanged by $p, q, \ldots$, and the term proportional to the field $D$ has changed sign. Note that the above Lagrangian does not represent the most general Lagrangian of this type. First of all (5.60) can be written in a form that is manifestly invariant under both the gauge transformations associated with the gauge fields $W_{\mu}{ }^{A}$ and the local $\mathrm{SU}(2)$ transformations, as follows from (5.53). Secondly, this Lagrangian is invariant under the $n+1$ rigid abelian transformations noted in (5.6). ${ }^{6}$ Both these properties are characteristic for dimensionally reduced $4 D$ vector multiplet Lagrangians and are not generic for these $3 D$ couplings.

Just as for the tensor multiplets (cf. (5.15)) a function $\mathcal{F}(x, v, \bar{v})$ should exist such that

$$
\frac{\partial^{2} \mathcal{F}(x, v, \bar{v})}{\partial x^{A} \partial x^{B}}=-\frac{\partial^{2} \mathcal{F}(x, v, \bar{v})}{\partial v^{A} \partial \bar{v}^{B}}=\mathcal{F}_{A B}, \quad \frac{\partial^{2} F(x, v, \bar{v})}{\partial x^{A} \partial v^{B}}=\frac{\partial^{2} F(x, v, \bar{v})}{\partial x^{B} \partial v^{A}} .
$$

The function $\mathcal{F}$ can be expressed in terms of the function $F(X)$ that encodes the $4 D$ vector multiplet Lagrangian and it takes the following form,

$$
\mathcal{F}(x, v, \bar{v})=-8 L^{0} \operatorname{Im}\left[\frac{F(X(L))}{\left(\bar{v}^{0}\right)^{2}}\right],
$$

where $X^{\Lambda}(L)$ is defined by (5.37). Clearly this function is homogeneous of degree +1 and it is also manifestly invariant under the shift transformations (5.6). Note, however, that it is not invariant under the full $\mathrm{SU}(2)$ R-symmetry group, but only under its $\mathrm{U}(1)$ subgroup. One can explicitly show that this function indeed satisfies the differential equations (5.61). Alternatively one can show that (5.62) satisfies the relation

$$
\chi_{\text {vector }}(L)=-\mathcal{F}(v, \bar{v}, x)+x^{A} \frac{\partial \mathcal{F}(x, v, \bar{v})}{\partial x^{A}},
$$

which is the exact analogue of (5.20). To prove this result we note the following useful equations,

$$
x^{A} \frac{\partial}{\partial x^{A}} \frac{F(X(L))}{\left(\bar{v}^{0}\right)^{2}}=-\frac{1}{2\left(L^{0}\right)^{2}}\left[\bar{X}^{\Lambda} F_{\Lambda}-\frac{\left|v^{0}\right|^{2}}{\left(\bar{v}^{0}\right)^{2}} X^{\Lambda} F_{\Lambda}\right],
$$

which follows upon using (5.39), (5.37) and (5.36).

\footnotetext{
${ }^{6}$ From the previous results in this subsection, the reader can verify that this is indeed the case. In fact the Lagrangian is expected to have more rigid symmetries but those are ignored here.
} 
Obviously the function (5.62) is singular when imposing the $\mathrm{SU}(2)$ gauge condition $v^{0}=0$. In that case we have $X^{\Lambda}=\frac{1}{2} \mathrm{i} \bar{v}^{\Lambda}$, and the role of the function (5.62) is taken over by a different function,

$$
\hat{\mathcal{F}}(x, v, \bar{v})=\frac{N_{\Lambda \Sigma}\left[x^{\Lambda} x^{\Sigma}-2 v^{\Lambda} \bar{v}^{\Sigma}\right]}{4 x^{0}}
$$

which satisfies the same equations (5.61) for $A, B=\Lambda, \Sigma$, as well as

$$
\frac{\partial^{2} \hat{\mathcal{F}}(x, v, \bar{v})}{\partial x^{0} \partial x^{0}}=\mathcal{F}_{00}
$$

In [11] the result (5.65) was noted in the case of rigid supersymmetry (where $x^{0}$ equals a constant) for the c-map between vector and tensor multiplets. It was later extended to local supersymmetry in $[12,13]$. Note, however, that the general context in [11-13] is somewhat different than in this paper as it is primarily directed towards the study of hypermultiplets.

Here we should add that different functions $\mathcal{F}(x, v, \bar{v})$ (as well as $\hat{\mathcal{F}}(x, v, \bar{v})$ ) will correspond to different Lagrangians that can, however, still describe the same theory, as we can deduce from the existence of electric-magnetic duality of the $4 D$ vector multiplet Lagrangians. An analogous situation exists for the $4 D$ tensor Lagrangians because of 'tensor-tensor' duality [11] (the existence of such tensor dualities is now also implied by the c-map).

\subsection{The c-map}

We have now determined the $3 D$ Lagrangian for systems of hypermultiplets, tensor multiplets and vector multiplets quadratic in space-time derivatives. As noted in subsection 5.2.1, there exist two different hypermultiplets, distinguished by the fact that their scalar sections, $A_{i}{ }^{\alpha}$ and $\tilde{A}_{p}{ }^{\alpha}$, transform under different $\mathrm{SU}(2)$ factors of the R-symmetry group. Their corresponding Lagrangians are given in (5.11) and (5.12). Let us then summarize the terms in the combined Lagrangian that contain the Ricci scalar, the two auxiliary fields of the superconformal multiplet, $C$ and $D$, as well as the kinetic terms of the scalars of the various supermultiplets,

$$
\begin{aligned}
e^{-1} \mathcal{L}= & \frac{1}{4}\left(\chi_{\text {hyper }}+\chi_{\text {tensor }}+\tilde{\chi}_{\text {hyper }}+\chi_{\text {vector }}\right)\left(\frac{1}{2} R-C^{2}\right) \\
& +\frac{1}{4}\left(\chi_{\text {hyper }}+\chi_{\text {tensor }}-\tilde{\chi}_{\text {hyper }}-\chi_{\text {vector }}\right) D \\
& -\frac{1}{2} \Omega_{\alpha \beta} \varepsilon^{i j} \mathcal{D}_{\mu} A_{i}{ }^{\alpha} \mathcal{D}^{\mu} A_{j}{ }^{\beta}-\frac{1}{2} \tilde{\Omega}_{\alpha \beta} \varepsilon^{i j} \mathcal{D}_{\mu} \tilde{A}_{i}{ }^{\alpha} \mathcal{D}^{\mu} \tilde{A}_{j}{ }^{\beta} \\
& +\frac{1}{2} F_{I J} \mathcal{D}_{\mu} L^{i}{ }_{j}{ }^{I} \mathcal{D}^{\mu} L^{j}{ }_{i}{ }^{J}+\frac{1}{2} \mathcal{F}_{A B} \mathcal{D}_{\mu} L^{p}{ }_{q}{ }^{A} \mathcal{D}^{\mu} L^{q}{ }_{p}{ }^{B}
\end{aligned}
$$


Here we made use of the vector and tensor potentials as well as the hyperkähler potentials, which are homogeneous in the scalar fields and invariant under R-symmetry,

$$
\begin{aligned}
\chi_{\text {hyper }} & =\frac{1}{2} \Omega_{\alpha \beta} \varepsilon^{i j} A_{i}{ }^{\alpha} A_{j}{ }^{\beta}, \\
\tilde{\chi}_{\text {hyper }} & =\frac{1}{2} \tilde{\Omega}_{\alpha \beta} \varepsilon^{p q} \tilde{A}_{p}{ }^{\alpha} \tilde{A}_{q}{ }^{\beta}, \\
\chi_{\text {tensor }} & =-2 F_{I J} L_{j}^{i}{ }^{I^{j}} L_{i}{ }^{J}, \\
\chi_{\text {vector }} & =-2 \mathcal{F}_{A B} L^{p}{ }_{q}{ }^{A} L_{p}^{q}{ }^{B} .
\end{aligned}
$$

The above equations represent the generic situation in three dimensions. ${ }^{7}$ It seems that there is a symmetric situation between the two sectors corresponding to ( $\chi_{\text {hyper }}+$ $\left.\chi_{\text {tensor }}\right)$ and $\left(\tilde{\chi}_{\text {hyper }}+\chi_{\text {vector }}\right)$, which involves also the reflection $(3.3)$ noted for the $3 D$ superconformal multiplet. While we have obtained these results by dimensional reduction from four dimensions, starting with vector and tensor multiplets and only one type of hypermultiplets, one may now consider the inverse procedure and ask which of these $3 D$ theories can be uplifted to four dimensions. A special subclass then consists of those theories that can be uplifted to $4 D$ in two different ways, meaning that the $3 D$ Lagrangian and its dual one with respect to the reflection (3.3) can both be uplifted. In that case there will exist two inequivalent $4 d$ Lagrangians that yield the same $3 D$ theory upon dimensional reduction. Henceforth we will concentrate on this subclass.

To have two possible uplifts, the $3 D$ Lagrangian must obviously satisfy a number of restrictions. As already explained, under dimensional reduction as carried out in this paper, the vector multiplet Lagrangian is of a restricted type. This implies that the alternative uplift to four space-time dimensions is only possible when also the tensor multiplet Lagrangian belongs to this restricted class. A similar argument applies to the hypermultiplets. Since the hypermultiplet Lagrangian associated with the hyperkähler potential $\tilde{\chi}_{\text {hyper }}$ cannot be obtained directly by dimensional reduction from $4 D$ hypermultiplets, it can only emerge via vector-scalar duality from the vector sector. Hence to have two alternative uplifts to $4 D$ the two hyperkähler Lagrangians should both be such that they can be obtained from scalar-vector duality from a restricted $3 D$ vector Lagrangian. When dualizing $n+2$ vector multiplets one obtains a hyperkähler cone of quaternionic dimension $n+2$ with $2 n+3$ tri-holomorphic abelian isometries. ${ }^{8}$

If one of the inequivalent $4 D$ Lagrangians has $n_{\mathrm{v}}$ (off-shell) vector multiplets and $n_{\mathrm{t}}$ (off-shell) tensor multiplets (ignoring the hypermultiplets for convenience), then the other uplift should have $n_{\mathrm{t}}-1$ (off-shell) vector and $n_{\mathrm{v}}+1$ (off-shell) tensor multiplets (so that the total number of off-shell vector and tensor multiplets in $3 D$ equals $\left.n_{\mathrm{v}}+n_{\mathrm{t}}+1\right)$. Obviously we have the condition that there must at least be one off-shell tensor supermultiplet in either one of the two inequivalent $4 D$ Lagrangians! The map between these two inequivalent $4 D$ theories is known as the c-map. From the perspective of the $10 D$ IIA and IIB

\footnotetext{
${ }^{7}$ For simplicity we are ignoring the option of partially performing vector-scalar dualities in which case one obtains an (on-shell) Lagrangian that consists of vector multiplets and hypermultiplets with mutual interactions beyond the ones induced by the coupling to the fields of the superconformal theory.

${ }^{8}$ Note that for the on-shell theory the corresponding quaternion-Kähler manifold of quaternionic dimension $n+1$ has only $n+2$ commuting quaternionic abelian isometries [3].
} 
supergravities compactified on the circle $S^{1}$ times a six-dimensional internal manifold that preserves eight supersymmetries, the resulting reduction to $3 D$ leads to a Lagrangian that can be uplifted to $4 D$ in two different ways. Those will then correspond to the compactified IIA and the compactified IIB theories. In string theory these two theories emerge in the compactification of type-II string theory on a circle in its two decompactification limits, where either the momentum modes or the winding modes become massless. Hence this property of lower-dimensional matter-coupled supergravities can thus be seen as a consequence of T-duality for type-II strings [1].

\section{The c-map for higher-derivative couplings}

The off-shell reduction scheme introduced in this paper can be straightforwardly applied to higher-derivative Lagrangians. Higher-derivative couplings in $4 D$ can be generated by coupling a number of vector multiplets to the Weyl multiplet (its covariant quantities constitute a chiral multiplet with the anti-selfdual tensor $T_{a b}{ }^{i j}$ as its lowest component, in the same way as the covariant quantities of the vector multiplet define a chiral multiplet with the holomorphic scalar $X$ as its lowest component), by means of a chiral invariant [28]. To consider a similar coupling on the tensor multiplet side is, however, more complicated, although this can be handled by the standard technique of making use of composite multiplets. For instance, one can write an off-shell vector multiplet in terms of off-shell tensor multiplets [5], or an off-shell tensor multiplet in terms of vector multiplets. Since these composite multiplets contain two derivatives, their substitution into a standard two-derivative Lagrangian will lead to four space-time derivatives. Another way to generalize higherderivative couplings is by making use of the so-called 'kinetic multiplet', which leads in principle to non-chiral invariants $[22,30,31]$.

For simplicity, we will first consider the Lagrangians derived in the previous section and replace some of the elementary vector and/or tensor multiplets by composite ones. In this way we will naturally obtain higher-derivative actions that can be uplifted to two different $4 D$ theories, which are thus related by the c-map. In the next subsection we will first introduce the key formulae for these composite multiplets. In the last subsection we will briefly consider the coupling to a composite chiral multiplet consisting of the square of the Weyl multiplet.

\subsection{Higher derivative couplings through composite matter multiplets}

In order to discuss higher-derivative actions for matter multiplets it is convenient to introduce some elements of the multiplet calculus known in four dimensions, which can be straightforwardly reduced to three dimensions, using the formulae in section 5.1.

In four dimensions, one may construct composite vector multiplets out of a set of tensor multiplets [5]. The starting point is the lowest-weight components of the vector multiplets, the complex scalars $X^{\text {comp }}$, which take the form

$$
X^{\mathrm{comp}}=f(L)_{I} \bar{G}^{I}+f(L)_{I J}^{i j} \bar{\varphi}_{i}^{I} \varphi_{j}^{J}
$$


where the $f(L)_{I}$ are functions of the tensor multiplet scalars $L_{i j}{ }^{I}$, which are homogeneous of degree -1 . The $f(L)_{I J}{ }^{i j}$ then denote their derivatives with respect to $L_{i j}{ }^{J}$, and $G^{I}$ and $\varphi^{I}$ denote the auxiliary fields and the spinor fields of the $4 D$ tensor multiplets. The functions $f_{I}$ are subject to two additional constraints, namely

$$
f_{I J}{ }^{i j}=f_{J I}^{i j}, \quad \varepsilon^{j k} \frac{\partial f_{I J i j}}{\partial L^{k l K}}=0 .
$$

These constraints are similar to the ones noted in subsection 5.2.2 for the function $F_{I J}$ that appears in the $4 D$ tensor multiplet action. In components their solution takes the form (5.15) upon suppressing the first index $I$. The functions $f(L)_{I}$ must be invariant under the $4 D \mathrm{SU}(2)$ R-symmetry group, so that the composite scalar $X^{\text {comp }}$ transforms as as a proper $4 D$ chiral multiplet scalar.

The remaining components of the composite multiplet are then identified straightforwardly upon considering consecutive supersymmetry variations. As an example we present the expression for the composite spinor associated with the composite vector multiplet,

$$
\begin{aligned}
\Omega_{i}^{\text {comp }}= & -2 f_{I}\left[\not D \varphi_{i}{ }^{I}+3 L_{i j}{ }^{I} \chi^{j}+\frac{1}{8} \varepsilon_{i j} T_{A B}{ }^{j k} \gamma^{A B} \varepsilon_{k l} \varphi^{l I}\right] \\
& +2 f_{I J i j} \bar{G}^{I} \varphi^{j J}-2 f_{I J}{ }^{k l}\left(\not D L_{i k}{ }^{I}-\varepsilon_{i k} \not^{I}\right) \varphi_{l}{ }^{J}+2 f_{I J K i j}{ }^{k l} \varphi^{j K} \bar{\varphi}_{k}{ }^{I} \varphi_{l}{ }^{J},
\end{aligned}
$$

where $f_{I J K i j}{ }^{k l}=\partial^{2} f_{I} / \partial L^{i j J} \partial L_{k l}{ }^{K}$.

Also the reverse situation is possible, and one may construct a four-dimensional composite tensor multiplets out of a set of vector multiplets. In this case, the lowest-weight component is an $\mathrm{SU}(2)$ triplet of scalars $L_{i j}^{\text {comp }}$, which is given by

$$
\begin{aligned}
L_{i j}^{\text {comp }}= & g(X)_{\Lambda} Y_{i j}{ }^{\Lambda}-\frac{1}{2} g(X)_{\Lambda \Sigma} \bar{\Omega}_{(i}{ }_{(i} \Omega_{j}{ }^{\Sigma} \\
& +\varepsilon_{i k} \varepsilon_{j l}\left[\bar{g}_{\Lambda}(\bar{X}) Y^{k l \Lambda}-\frac{1}{2} \bar{g}(\bar{X})_{\Lambda \Sigma} \bar{\Omega}^{(k \Lambda \Lambda} \Omega^{l) \Sigma}\right],
\end{aligned}
$$

where the $g_{\Lambda}(X)$ are holomorphic functions of the vector multiplet scalars $X^{\Lambda}$ which are homogeneous of zeroth degree. The $g_{\Lambda \Sigma}$ denote the derivative of $g_{\Lambda}$ with respect to $X^{\Sigma}$. Again there is a constraint on the derivatives of the functions $g_{\Lambda}$,

$$
g_{\Lambda \Sigma}=g_{\Sigma \Lambda}
$$

which implies that the $g_{\Lambda}$ can be expressed in terms of a derivative of a holomorphic function, $g_{\Lambda}=\partial g / \partial X^{\Lambda}$. Just as before the remaining components of this multiplet will follow from applying consecutive supersymmetry variations of (6.4). As an example we present the corresponding expression for the composite spinor,

$$
\begin{aligned}
\varphi_{i}^{\text {comp }}= & \left(g_{\Lambda}+\bar{g}_{\Lambda}\right) \not D \Omega_{i}{ }^{\Lambda}-\not D g_{\Lambda} \Omega_{i}{ }^{\Lambda} \\
& +\frac{1}{2} \bar{g}_{\Lambda \Sigma} Y_{i j}{ }^{\Lambda} \Omega^{j \Sigma}-\frac{1}{4} \varepsilon_{i j} \bar{g}_{\Lambda \Sigma}\left(F_{A B}{ }^{\Lambda}-\frac{1}{4} X T_{A B k l} \varepsilon^{k l}\right) \gamma^{A B} \Omega^{j \Sigma} \\
& +\frac{1}{64} \bar{g}_{\Lambda \Sigma \Xi} \varepsilon_{i j} \gamma_{A B} \Omega^{j \Lambda} \varepsilon_{k l} \bar{\Omega}^{k \Sigma} \gamma^{A B} \Omega^{l \Xi} .
\end{aligned}
$$


The underlying reason why this construction works is related to the fact that the equations of motion associated with a (two-derivative) vector multiplet Lagrangian transform as a tensor multiplet, and vice versa. This remains true in a superconformal background. The conditions (6.2) and (6.5) can be understood in this perspective: when these conditions hold one can construct invariant Lagrangians based on such functions.

With the above results one can in principle obtain the corresponding $3 D$ composite multiplets by applying the dictionary given in section 5.1 to all the bosonic components of the composite multiplets.

Starting from the composite vector multiplet defined by (6.1), we write both sides of the equations in terms of the corresponding components of the $3 D$ multiplets. The relevant functions $f(L)_{I}$ and $g_{\Lambda}$ are then written in terms of the proper $3 D$ fields. The $f_{I}$ are written in terms of the $3 D$ of the $L^{i}{ }_{j}{ }^{I}$, after extracting a uniform factor $1 /\left(2 L^{0}\right)$,

$$
f\left(L^{4 D}\right)_{I} \longrightarrow \frac{1}{2 L^{0}} f\left(L^{3 D}\right)_{I}
$$

The new function thus remains $\mathrm{SU}(2)$ invariant and homogeneous of degree -1 in the $3 D$ scalars. This is all dictated by the off-shell dictionary (see, in particular, (5.4) and (5.7)). It is then straightforward to obtain the following results for the bosonic composite vector multiplet components (suppressing their fermionic contributions),

$$
\begin{aligned}
& L_{q}^{p \operatorname{comp}}=f(L)_{I} Y_{q}^{p}, \\
& F(W)_{\mu \nu}{ }^{\text {comp }}=f_{I J i}{ }^{j} \mathcal{D}_{[\mu} L^{i}{ }_{k}^{I} \mathcal{D}_{\nu]} L^{k}{ }_{j}{ }^{J}-\frac{1}{2} f_{I} L^{i}{ }_{j}^{I} R(\mathcal{V})_{\mu \nu}{ }^{j}{ }_{i}+\partial_{[\mu}\left[\mathrm{i} e^{-1} \varepsilon_{\nu] \rho \sigma} f_{I} F(E)^{\rho \sigma I}\right], \\
& Y_{j}^{i}{ }_{j}^{\mathrm{comp}}=f_{I}\left[\mathcal{D}^{2} L^{i}{ }_{j}+\frac{1}{2}\left(\frac{1}{2} R+D-C^{2}\right) L^{i I}\right]+f_{I J}{ }_{l}{ }_{l} \mathcal{D}_{\mu} L_{k}^{l}{ }^{I} \mathcal{D}^{\mu} L^{i}{ }_{j} \\
& +\frac{1}{2} f_{I J}{ }^{i}{ }_{j}\left[Y^{p}{ }_{q}^{I} Y^{q}{ }_{p}^{J}+F(E)^{a b I} F(E)_{a b}{ }^{J}-\mathcal{D}_{\mu} L^{k}{ }_{l}^{I} \mathcal{D}^{\mu} L_{k}^{l}{ }_{k}\right] \\
& +\frac{1}{2} \mathrm{i} \varepsilon^{\mu \nu \rho}\left[f_{I J^{i}{ }_{k}} \mathcal{D}_{\mu} L^{k}{ }_{j}^{I}-f_{I J}{ }_{j}{ }_{j} \mathcal{D}_{\mu} L^{i}{ }_{k}^{I}\right] F(E)_{\nu \rho}{ }^{J} .
\end{aligned}
$$

The derivation for the composite tensor multiplet proceeds along similar lines, except that the Kaluza-Klein vector multiplet will now also contribute, Hence the sum over the vector multiplets in (6.4) and (6.6) will now include an extra vector multiplet. The function $g$ is written in terms of the fields $L^{p}{ }_{q}^{A}$ (thus including the Kaluza-Klein scalar). The degree of homogeneity is changed because we have to absorb a factor $1 / L^{0}$. This is all dictated by the off-shell dictionary (see, in particular, (5.4) and (5.7)). It is then straightforward to obtain the conversion to

$$
[g(X)+\bar{g}(\bar{X})]_{\Lambda} \longrightarrow \frac{1}{L^{0}} g\left(L^{3 D}\right)_{A}
$$

with $A=\Lambda, 0$ and where

$$
g\left(L^{3 D}\right)_{A}=\left\{\begin{array}{l}
g\left(L^{3 D}\right)_{\Lambda} \\
g\left(L^{3 D}\right)_{\Sigma} \frac{L^{p} q^{\Sigma} L^{q} p^{0}}{2\left(L^{0}\right)^{2}}
\end{array}\right.
$$


As a consequence of (6.10) the resulting expressions will again be invariant under the transformations (5.6). We now present the bosonic components of the composite tensor multiplet, converted to $3 D$ and suppressing fermionic contributions,

$$
\begin{aligned}
& L_{j}^{i \text { comp }}=g(L)_{A} Y_{j}^{i}, \\
& F(E)_{\mu \nu}{ }^{\mathrm{comp}}=g_{A B p}{ }^{q} \mathcal{D}_{[\mu} L_{r}^{p}{ }^{A} \mathcal{D}_{\nu]} L_{q}^{r}{ }_{q}^{B}-\frac{1}{2} g_{A} L_{q}^{p}{ }_{q}^{A} R(\mathcal{A})_{\mu \nu}{ }_{p}+\partial_{[\mu}\left[\mathrm{i} e^{-1} \varepsilon_{\nu] \rho \sigma} g_{A} F(W)^{\rho \sigma A}\right] \text {, } \\
& Y_{q}^{p \text { comp }}=g_{A}\left[\mathcal{D}^{2} L_{q}^{p}{ }_{q}^{A}+\frac{1}{2}\left(\frac{1}{2} R-D-C^{2}\right) L_{q}^{p A}\right]+g_{A B}{ }^{r}{ }_{s} \mathcal{D}_{\mu} L_{r}^{s}{ }^{A} \mathcal{D}^{\mu} L_{q}^{p B} \\
& +\frac{1}{2} g_{A B}{ }_{q}\left[Y^{i}{ }_{j}^{A} Y^{j}{ }_{i}^{B}+F(W)^{a b A} F(W)_{a b}{ }^{B}-\mathcal{D}_{\mu} L^{r}{ }_{s}^{A} \mathcal{D}^{\mu} L^{s}{ }_{r}^{B}\right] \\
& +\frac{1}{2} \mathrm{i} \varepsilon^{\mu \nu \rho}\left[g_{A B}{ }_{r} \mathcal{D}_{\mu} L^{r}{ }_{q}^{A}-g_{A B}{ }^{r} \mathcal{D}_{\mu} L^{p}{ }_{r}^{A}\right] F(W)_{\nu \rho}{ }^{B} .
\end{aligned}
$$

The components of the composite vector and tensor multiplets clearly share a common structure. Apart from the fact that the indices are different (because they transform under a different $\mathrm{SU}(2)$ factor of the R-symmetry group) and that an additional Kaluza-Klein vector multiplet has emerged in the composite tensor multiplet, the only obvious difference is that the field $D$ appears with opposite signs in (6.8) and (6.11), which is consistent with the reflection symmetry noted in (3.3). However, there is also another, more implicit, difference associated with the field strengths $F(W)_{\mu \nu}{ }^{\text {comp }}$ and $F(E)_{\mu \nu}{ }^{\text {comp }}$. One can show that both of them satisfy a Bianchi identity, which implies that there should exist explicit expressions for the corresponding composite gauge fields $W_{\mu}^{\text {comp }}$ and $E_{\mu}^{\text {comp }}$. However, as we have already noted when discussing the Lagrangians with two derivatives in subsection 5.2, the expression for $W_{\mu}^{\text {comp }}$ is in general not invariant under the relevant $\mathrm{SU}(2)$ R-symmetry, whereas the expression for $E_{\mu}^{\text {comp }}$ will be manifestly invariant under the relevant $\mathrm{SU}(2)$. This should not come as a surprise in view of the fact that the composites can be associated with the field equations belonging to some appropriately chosen Lagrangian. Since $F(E)_{\mu \nu}{ }^{\text {comp }}$ is therefore a field equation belonging to a vector multiplet Lagrangian, $E_{\mu}{ }^{\text {comp }}$ will thus be manifestly $\mathrm{SU}(2)$ invariant. For $W_{\mu}^{\text {comp }}$ the situation is different and it will not necessarily be $\mathrm{SU}(2)$ invariant. Whether or not this is the case will depend on the functions $f(L)_{I}$ that one intends to use.

There is also another feature that is relevant, namely, as was already alluded to above, the composite tensor multiplet components (6.8) will necessarily be invariant under the transformations (5.6), whereas the vector multiplet components will in general not be subject to such a symmetry. Hence consistency with the c-map will requires that the functions $f(L)_{I}$ will satisfy such a symmetry as well. Provided that this is the case, one may construct the actions for vectors and tensors by including both the elementary and a number of composite multiplets in the way that was described in section 5.2, because from this construction there is no difference between elementary and composite multiplets. One starts from a $4 D$ Lagrangian describing $n_{\mathrm{v}}$ elementary and $\tilde{n}_{\mathrm{v}}$ composite vector multiplets (the latter described in terms of $n_{\mathrm{t}}$ elementary tensor multiplets), and a second Lagrangian describing $n_{\mathrm{t}}$ elementary tensor and $\tilde{n}_{\mathrm{t}}$ composite tensor multiplets (the latter expressed in terms of the $n_{\mathrm{v}}$ elementary vector multiplets). This then leads to a $3 D$ action which, under the conditions described above, can then also be uplifted to the sum of two $4 D$ 
Lagrangians, one describing $n_{\mathrm{t}}-1$ elementary and $\tilde{n}_{\mathrm{t}}$ composite vector multiplets (the latter described in terms of $n_{\mathrm{v}}+1$ elementary tensor multiplets), and a second one based on $n_{\mathrm{v}}+1$ elementary and $\tilde{n}_{\mathrm{v}}$ composite tensor multiplets (the latter expressed in terms of the $n_{\mathrm{t}}-1$ elementary vector multiplets).

We refrain from working out some of these theories in detail and leave this to later work. It is clear that, by considering composite multiplets that themselves depend on both elementary and composite multiplets, one can successively construct interactions that will involve even higher-order derivatives. In our next and last subsection we will briefly discuss other higher-derivative Lagrangians and describe some details about their reduction to three dimensions.

\subsection{More higher-derivative couplings}

As is well-known there exists a larger class of $4 D$ higher-derivative actions for vector supermultiplets, possibly involving the Weyl multiplet $[22,28,30]$. The latter is a reduced chiral tensor multiplet, whose lowest-weight component equals $\varepsilon_{i j} T_{A B}{ }^{i j}$. In all cases it is the square of the Weyl multiplet that enters, so that the resulting multiplet is a composite chiral multiplet whose lowest-weight component equals the composite scalar $\hat{A}=\left(\varepsilon_{i j} T_{A B}{ }^{i j}\right)^{2}$. For the subsequent discussion we also present the bosonic contributions to the highestweight component of this multiplet, which is denoted by $\hat{C}$,

$$
\begin{aligned}
\hat{C}= & 64 \mathcal{R}(M)^{-C D}{ }_{A B} \mathcal{R}(M)_{C D}^{-}{ }^{A B}+32 R(\mathcal{V})^{-A B i}{ }_{j} R(\mathcal{V})_{A B}^{-}{ }^{j}{ }_{i} \\
& -32 T^{A B i j} D_{A} D^{C} T_{C B i j},
\end{aligned}
$$

where $\mathcal{R}(M)^{-C D}{ }_{A B}$ is a generalization of the (anti-selfdual component) of the Weyl tensor. Since this composite multiplet is a scalar chiral multiplet, it can be directly coupled to vector multiplets as well as to (composite) tensor multiplets. A full discussion of these couplings is outside the scope of the present paper, and here we will mainly confine ourselves to a partial analysis of square of the the Weyl multiplet upon its reduction to three dimensions.

Using the dictionary in subsection 5.1 we can express the components $\hat{A}$ and $\hat{C}$ in terms of $3 D$ fields (suppressing fermionic contributions),

$$
\begin{aligned}
\hat{A}= & -\frac{4}{\left(L^{0}\right)^{4}}\left[\left(\bar{v}^{0} \stackrel{\leftrightarrow}{\mathcal{D}}_{a} x^{0}\right)-\frac{\bar{v}^{0}}{L^{0}+\frac{1}{2} x^{0}}\left(v^{0} \stackrel{\leftrightarrow}{\mathcal{D}}_{a} \bar{v}^{0}\right)\right]^{2}, \\
\hat{C}= & 32\left[R^{\mu \nu} R_{\mu \nu}-\frac{3}{8} R^{2}\right]+64\left(\mathcal{D}_{\mu} C\right)^{2}+48 D^{2} \\
& +16\left[R(\mathcal{V})^{\mu \nu i}{ }_{j} R(\mathcal{V})_{\mu \nu}{ }_{i}+2 R(\mathcal{A})^{\mu \nu p}{ }_{q} R(\mathcal{A})_{\mu \nu}{ }^{q}{ }_{p}\right] \\
& +\frac{32}{\left(L^{0}\right)^{2}}\left[\mathcal{D}^{\mu} F^{\nu \rho 0} \mathcal{D}_{\mu} F_{\nu \rho}{ }^{0}-2 \mathcal{D}_{\mu} F^{\mu \nu 0} \mathcal{D}^{\rho} F_{\rho \nu}{ }^{0}\right] \\
& +\frac{32}{\left(L^{0}\right)^{2}}\left[\mathcal{D}_{\mu} Y^{i}{ }_{j}^{0} \mathcal{D}^{\mu} Y^{j}{ }_{i}^{0}+\mathrm{i} e^{-1} \varepsilon^{\mu \nu \rho} L^{0} R(\mathcal{V})_{\mu \nu}{ }_{j}{ }_{j} \mathcal{D}_{\rho} Y^{j}{ }_{i}{ }^{0}\right]+\cdots,
\end{aligned}
$$

where in $\hat{C}$ we restricted ourselves to only some characteristic terms. 
To elucidate this result let us consider the bosonic terms of the $4 D$ superconformal action, which can be written as

$$
\left.\mathcal{L}^{\text {s.c. }}\right|_{4 D}=E\left[\hat{C}-\frac{1}{16} \hat{A}\left(T_{A B i j} \varepsilon^{i j}\right)^{2}\right]+\text { h.c. },
$$

where the second term represents the bosonic contribution of the chiral superspace measure. Upon its reduction to three dimensions, we write the result as a linear combination of two terms,

$$
\left.e^{-1} \mathcal{L}^{\text {s.c. }}\right|_{3 D}=e^{-1} \mathcal{L}_{1}+e^{-1} \mathcal{L}_{2}
$$

with

$$
\begin{aligned}
e^{-1} \mathcal{L}_{1}= & \frac{64}{L^{0}}\left[R^{\mu \nu} R_{\mu \nu}-\frac{3}{8} R^{2}+\frac{1}{2} R(\mathcal{V})^{\mu \nu i}{ }_{j} R(\mathcal{V})_{\mu \nu}{ }^{j}{ }_{i}+R(\mathcal{A})^{\mu \nu p}{ }_{q} R(\mathcal{A})_{\mu \nu}{ }_{p}{ }_{p}\right. \\
& \left.+2\left(\mathcal{D}_{a} C\right)^{2}+\frac{3}{2}\left(D-C^{2}\right)^{2}\right]
\end{aligned}
$$

and

$$
\begin{aligned}
e^{-1} \mathcal{L}_{2}= & \frac{64}{\left(L^{0}\right)^{3}}\left[\mathcal{D}^{\mu} F^{\nu \rho 0} \mathcal{D}_{\mu} F_{\nu \rho}{ }^{0}-2 \mathcal{D}_{\mu} F^{\mu \nu 0} \mathcal{D}^{\rho} F_{\rho \nu}{ }^{0}\right. \\
& +\mathcal{D}_{\mu} Y^{i}{ }_{j}^{0} \mathcal{D}^{\mu} Y^{j}{ }_{i}^{0}+i e^{-1} \varepsilon^{\mu \nu \rho} L^{0} R(\mathcal{V})_{\mu \nu}{ }_{j}{ }_{j} \mathcal{D}_{\rho} Y^{j}{ }_{i}{ }^{0} \\
& \left.+\frac{1}{4}\left(C^{2}-D\right)\left(3 Y^{i}{ }_{j}^{0} Y^{j}{ }_{i}^{0}+\left(F_{\mu \nu}{ }^{0}\right)^{2}\right)\right] \\
& +\frac{4}{\left(L^{0}\right)^{5}}\left[3\left(F_{\mu \nu}{ }^{0}\right)^{2}\left(F_{\rho \sigma}{ }^{0}\right)^{2}+3\left(Y^{i}{ }_{j}^{0} Y^{j}{ }_{i}^{0}\right)^{2}+2 Y_{j}^{i}{ }_{j}^{0} Y^{j}{ }_{i}^{0}\left(F_{\mu \nu}{ }^{0}\right)^{2}\right]+\cdots
\end{aligned}
$$

We should emphasize that the above expressions concern only a subset of the terms generated by the reduction and are thus incomplete. As we have already seen in section 5.2.3, where we evaluated the $3 D$ Lagrangian for vector multiplets, a full evaluation of the $3 D$ results can be rather tedious and this is particularly the case for Lagrangians with higherderivative couplings. Nevertheless the above results already show a number of noteworthy features that will be present in the final result. Those will be briefly discussed below.

First of all this Lagrangian depends on both the fields of the $3 D$ Weyl multiplet and of the Kaluza-Klein vector multiplet. Clearly it is homogeneous of degree -1 in the latter fields, and the super conformal fields appear in a non-linear fashion. This can be understood on more general grounds, just as it was clear from the start that the Lagrangian should contain fourth-order space-time derivatives.

The Lagrangian $\mathcal{L}_{1}$ contains terms that are familiar from previous work on higherderivative Lagrangians for $3 D$ (super)gravity, multiplied by a compensating $\left(L^{0}\right)^{-1}$ factor that is required by conformal invariance. The linearized result for the corresponding supergravity invariant was given in [16] and exhibits all the quadratic bosonic terms present in (6.16). However, there are some notable differences in the coefficients. One is that the squares of the two $\mathrm{SU}(2)$ curvatures appear with different coefficients, unlike in [16] where the coefficients are the same. The other one concerns the coefficient of the kinetic term of the field $C$, which is positive. This discrepancy in the coefficients is no reason for 
concern: additional curvature terms may arise by commutators of covariant derivatives, and the scalar kinetic terms are effected by the presence of additional terms, for instance proportional to $\mathcal{D}_{\mu} L^{0} \mathcal{D}^{\mu} C$, that we have not extracted but that will change the coefficient of the $\left(\mathcal{D}_{\mu} C\right)^{2}$. Obviously the terms shown in (6.17) have no bearing on the expression in [16], because the presence of the components of the Kaluza-Klein vector multiplet is even more crucial here. It should be of interest to evaluate the full $3 D$ superconformal invariant, either from the off-shell dimensional reduction or directly in three space-time dimensions. The latter can be done by utilizing the off-shell multiplet calculus obtained in [19, 20].

The $4 D$ Weyl multiplet can easily be coupled to vector multiplets. Schematically one has a function $F(X, \hat{A})$, which can for instance be expanded in positive powers of $\hat{A}$ according to

$$
F(X, \hat{A})=\sum_{g} F_{g}(X) \hat{A}^{g}
$$

where each holomorphic function, $F_{g}(X)$ is of appropriate weight to ensure consistency with respect to conformal invariance. Comparing with (6.13), where $\hat{A}$ is expressed in terms of derivatives of the compensating scalars, it is clear that each term in (6.18) contributes $2 g$ first-order derivatives on the scalars. This can be compared to the situation in $4 D$, where the off-shell Lagrangian contains only four-derivative interactions, while a similar series of ever increasing derivatives appears when solving for the auxiliary tensor $T_{A B}{ }^{i j}$.

Finally we emphasize that we have only briefly considered the coupling of the Weyl multiplet to vector multiplets in this section. There also exist couplings that involve tensor multiplets. Those will of course be relevant for establishing consistency with the c-map. Assuming that this can be achieved, it may further clarify the effective action description for topological amplitudes involving tensor multiplets or hypermultipets [14].

\section{Acknowledgments}

We thank Nathan Berkovits, Daniel Butter, Martin Roček, Warren Siegel and Stefan Vandoren for valuable discussions. N.B. acknowledges the hospitality extended to her at Nikhef where her work was supported by a Veni grant of the 'Nederlandse Organisatie voor Wetenschappelijk Onderzoek (NWO)'. At present her work is partially supported by a Ramanujan Fellowship, DST, Government of India. B.d.W. is supported by the ERC Advanced Grant no. 246974, "Supersymmetry: a window to non-perturbative physics". The work of S.K. is supported by the ERC Starting Grant no. 307286, "The structure of the extra dimensions of string theory" and by the INFN.

\section{A Relations between 4D and 3D Riemann curvatures}

Based on (2.5) one can evaluate the relation between $4 D$ and $3 D$ curvature components. In the equations below, derivatives $\mathcal{D}_{a}$ are covariant with respect to $3 D$ local Lorentz transformations and dilatations. The results are as follows (in this appendix the $4 D$ curvature 
components are consistently denoted by $\hat{R})$,

$$
\begin{aligned}
\hat{R}_{\mu \nu}^{a b}= & R_{\mu \nu}^{a b}+\frac{1}{2} \phi^{-2}\left[F(B)_{\mu}^{[a} F(B)_{\nu}{ }^{b]}+F(B)_{\mu \nu} F(B)^{a b}\right] \\
& -B_{[\mu}\left[2 \phi^{-3} F(B)_{\nu]}{ }^{[a} \mathcal{D}^{b]} \phi+\mathcal{D}_{\nu]}\left[\phi^{-2} F(B)^{a b}\right]\right] \\
\hat{R}_{\mu \nu}{ }^{a 4}= & -\mathcal{D}_{[\mu}\left[\phi^{-1} F(B)_{\nu]}^{a}\right]-\phi^{-2} \mathcal{D}^{a} \phi F(B)_{\mu \nu} \\
& +B_{[\mu}\left[2 \mathcal{D}_{\nu]}\left[\phi^{-2} \mathcal{D}^{a} \phi\right]+\frac{1}{2} \phi^{-3} F(B)_{\nu] b} F(B)^{a b}\right], \\
\hat{R}_{\mu \hat{4}}^{a b}= & \frac{1}{2} \mathcal{D}_{\mu}\left[\phi^{-2} F(B)^{a b}\right]+\phi^{-3} F(B)_{\mu}{ }^{[a} \mathcal{D}^{b]} \phi \\
\hat{R}_{\mu \hat{4}}^{a 4}= & -\mathcal{D}_{\mu}\left[\phi^{-2} \mathcal{D}^{a} \phi\right]-\frac{1}{4} \phi^{-3} F(B)_{\mu b} F(B)^{a b} .
\end{aligned}
$$

With tangent-space indices, $\hat{R}_{C D}{ }^{A B}$ takes the form,

$$
\begin{aligned}
\hat{R}_{c d}{ }^{a b} & =R_{c d}{ }^{a b}+\frac{1}{2} \phi^{-2}\left[F(B)_{c}{ }^{[a} F(B)_{d}{ }^{b]}+F(B)_{c d} F(B)^{a b}\right], \\
\hat{R}_{c d}{ }^{a 4} & =\frac{1}{2} \phi^{-1} \mathcal{D}^{a} F(B)_{c d}-\phi^{-2}\left[\mathcal{D}^{a} \phi F(B)_{c d}-F(B)^{a}{ }_{[c} \mathcal{D}_{d]} \phi\right], \\
\hat{R}_{c 4}{ }^{a b} & =\frac{1}{2} \phi^{-1} \mathcal{D}_{c} F(B)^{a b}-\phi^{-2}\left[F(B)^{a b} \mathcal{D}_{c} \phi-F(B)_{c}{ }^{[a} \mathcal{D}^{b]} \phi\right], \\
\hat{R}_{c 4}{ }^{a 4} & =-\phi D_{c}(\omega)\left[\phi^{-2} \mathcal{D}^{a} \phi\right]-\frac{1}{4} \phi^{-2} F(B)_{c b} F(B)^{a b} .
\end{aligned}
$$

Note that these components satisfy the pair-exchange property of the Riemann tensor. Contracted versions of the Riemann tensor take the form,

$$
\begin{aligned}
\hat{R}_{c B}{ }^{a B} & =R_{c b}{ }^{a b}+\frac{1}{2} \phi^{-2} F(B)_{c b} F(B)^{a b}-\phi \mathcal{D}_{c}\left[\phi^{-2} \mathcal{D}^{a} \phi\right], \\
\hat{R}_{A 4}{ }^{A b} & =\frac{1}{2} \phi^{-1} \mathcal{D}_{a} F(B)^{a b}-\frac{3}{2} \phi^{-2} F(B)^{a b} \mathcal{D}_{a} \phi, \\
\hat{R}_{A 4}{ }^{A 4} & =-\phi \mathcal{D}_{a}(\omega)\left[\phi^{-2} \mathcal{D}^{a} \phi\right]-\frac{1}{4} \phi^{-2} F(B)_{a b} F(B)^{a b}, \\
\hat{R}_{A B}{ }^{A B} & =R_{a b}{ }^{a b}-2 \phi \mathcal{D}_{a}\left[\phi^{-2} \mathcal{D}^{a} \phi\right]+\frac{1}{4} \phi^{-2} F(B)_{a b} F(B)^{a b} .
\end{aligned}
$$

Furthermore one may consider the components of $\hat{R}_{[A B}^{E F} \hat{R}_{C D] E F}$,

$$
\begin{aligned}
\hat{R}_{[a b}{ }^{E F} \hat{R}_{c d] E F}= & 0 \\
\hat{R}_{\hat{4}[a}{ }^{E F} \hat{R}_{c d] E F}= & -\phi \mathcal{D}_{[a}\left[\frac{1}{2} \phi^{-2} R_{c d]}{ }^{e f} F(B)_{e f}\right. \\
& +\frac{1}{8} \phi^{-4}\left[F(B)^{2} F(B)_{c d]}+2 F(B)^{e f} F(B)_{c e} F(B)_{d f}\right] \\
& \left.-2 \phi^{-1} F(B)_{c}^{e} \mathcal{D}_{d]}\left(\mathcal{D}_{e} \phi^{-1}\right)+F(B)_{c d]}\left(\mathcal{D} \phi^{-1}\right)^{2}\right] .
\end{aligned}
$$

where we made use of the Bianchi identity on $F(B)$ in the $3 D$ Riemann tensor. 


\section{B The conversion of $4 D$ chiral to $\mathrm{SU}(2) \times \mathrm{SU}(2)$ covariant $3 D$ spinors}

The original $4 D$ theory contains doublets of Majorana spinors that transform under the chiral R-symmetry group $\mathrm{SU}(2) \times \mathrm{U}(1)$. Hence they transform irreducibly according to the pseudo-real $(4,2)_{\mathrm{c}}$ representation of $\operatorname{Spin}(3,1) \times \mathrm{SU}(2) \times \mathrm{U}(1)$, where the subscript denotes the chiral $\mathrm{U}(1)$ charge. When reducing to three dimensions, a $4 D$ spinor decomposes into two real $3 D$ spinors and, as we shall demonstrate, the U(1) component of the R-symmetry will then extend to a second $\mathrm{SU}(2)$ group, so that we obtain a pseudoreal irreducible representation $(2,2,2)$ of $\operatorname{Spin}(2,1) \times \mathrm{SU}(2) \times \mathrm{SU}(2)$. To obtain the spinor fields in their $3 D$ form, we must convert the $4 D$ spinors such that the $3 D$ symmetry assignments become manifest. This conversion is the topic of this section where we will base ourselves on previous results presented in $[4,5]$

The analysis starts from the underlying Clifford algebra for the $4 D$ gamma matrices, which has to be defined such that they act reducibly on the original spinor. We remind the reader that the reduction amounts to compactifying the fourth coordinate $\hat{x}^{4}$ on a circle which is subsequently shrunk to zero size. The proper $3 D$ gamma matrices are now defined in terms of the $4 D$ gamma matrices by

$$
\hat{\gamma}^{a}=\gamma^{a} \tilde{\gamma}, \quad \text { where } \quad \tilde{\gamma}=-\mathrm{i} \gamma_{4} \gamma_{5} .
$$

The hermitian matrices $\tilde{\gamma}, \gamma_{4}$ and $\gamma_{5}$ are mutually anti-commuting, and square to the unit matrix. Furthermore they commute with the $\hat{\gamma}^{a}$. Hence we have obtained two mutually commuting three-dimensional Clifford algebras, generated by the $\hat{\gamma}^{a}$ and by $\left(\tilde{\gamma}, \gamma_{4}, \gamma_{5}\right)$, respectively. Observe that we have the identity,

$$
\hat{\gamma}^{[a} \hat{\gamma}^{b} \hat{\gamma}^{c]}=\mathrm{i} \varepsilon^{a b c} \mathbf{1}
$$

showing that the two separate $3 D$ Lorentz spinors into which a generic $4 D$ spinor decomposes transform according in the same Clifford algebra representation. Starting from a single $4 D$ spinor one thus obtains a doublet of $3 D$ spinors transforming under an extended R-symmetry group $\mathrm{SU}(2)$ with generators $\left(\tilde{\gamma}, \gamma_{4}, \gamma_{5}\right)$, subject to $\tilde{\gamma} \gamma_{4} \gamma_{5}=\mathrm{i} 1$. Obviously the generator proportional to $\gamma_{5}$ corresponds to the generator of chiral U(1) R-symmetry that is already present in $4 D$.

As a result of the redefinition of the $3 D$ gamma matrices, the definition of the Dirac conjugate will change, and consequently also the $4 D$ charge conjugation matrix must be redefined. The new Dirac conjugate and the new charge conjugation matrix read,

$$
\hat{C}=C \tilde{\gamma}, \quad \hat{\bar{\psi}}=\bar{\psi} \tilde{\gamma}
$$

Note, however, that it is still possible to further modify the charge conjugation matrix. Indeed, the $\mathrm{SU}(2) \times \mathrm{SU}(2)$ covariant $3 D$ spinor basis that we are about to construct will require such a modification. Based on the present redefinitions one easily verifies the following equations (using the properties of the charge conjugation matrix $C$ in $4 D$ ),

$$
\begin{aligned}
& \hat{C} \hat{\gamma}^{a} \hat{C}^{-1}=-\hat{\gamma}^{a \mathrm{~T}}, \quad \hat{C}^{\mathrm{T}}=-\hat{C}, \\
& \hat{C} \gamma_{4} \hat{C}^{-1}=\gamma_{4}^{\mathrm{T}}, \quad \hat{C} \tilde{\gamma} \hat{C}^{-1}=\tilde{\gamma}^{\mathrm{T}}, \quad \hat{C} \gamma_{5} \hat{C}^{-1}=-\gamma_{5}^{\mathrm{T}} .
\end{aligned}
$$


Now we have to extend the previous analysis to the case of a doublet of $4 D$ Majorana fermions. It is convenient to still express the fermions in terms of $4 D$ chiral components, because those transform systematically under the action of the SU(2) R-symmetry group that is manifest in $4 D$. The new $\mathrm{SU}(2)$ group that emerges in the reduction to $3 D$ as an extension of the $4 D$ chiral $\mathrm{U}(1)$ group, will commute with the original $4 D$ chiral $\mathrm{SU}(2)$. To study the way in which the two $\mathrm{SU}(2)$ factors are realized, let us start from a positivechirality $4 D$ spinor $\psi^{i}$ with $\mathrm{U}(1)$ charge $+1 / 2$, which we combine with the negative-chirality conjugate $\psi_{i}$, which is provided by the $4 D$ Majorana condition. The latter spinor thus has $\mathrm{U}(1)$ charge equal to $-1 / 2$, respectively. Since the spinors transform uniformly under the $3 D$ Lorentz transformations we will only be concerned with the possible R-symmetry transformations. Observing that the symmetry enhancement of the R-symmetry group will be based on the generators $\left(\tilde{\gamma}, \gamma_{4}, \gamma_{5}\right)$ identified before, one expects that the extended symmetry involves the following infinitesimal variation,

$$
\delta\left(\begin{array}{c}
\psi^{i} \\
\psi_{i}
\end{array}\right)=\frac{1}{2}\left(\begin{array}{cc}
\Lambda^{i}{ }_{j}+\mathrm{i} \alpha \delta_{j}^{i} & -\beta \gamma_{4} \varepsilon^{i j} \\
-\bar{\beta} \gamma_{4} \varepsilon_{i j} & \Lambda_{i}{ }^{j}-\mathrm{i} \alpha \delta_{i}{ }^{j}
\end{array}\right)\left(\begin{array}{c}
\psi^{j} \\
\psi_{j}
\end{array}\right),
$$

where $\Lambda_{j}^{i}$ is an anti-hermitian traceless matrix, i.e. it satisfies the relations,

$$
\Lambda_{i}^{i}=0 \quad \Lambda_{k}^{i} \varepsilon^{k j}+\Lambda_{k}^{j} \varepsilon^{i k}=0, \quad \Lambda_{i}^{j} \equiv\left(\Lambda^{i}{ }_{j}\right)^{*},
$$

and $\alpha, \beta$ and $\bar{\beta}$ are the transformation parameters of the new $\mathrm{SU}(2)$. The normalization of these parameters is of no concern at this point. The reader can directly verify that these transformations form a group and that the new $\mathrm{SU}(2)$ group commutes with the original one generated by the matrix $\Lambda_{j}^{i}$.

The representation (B.5) has the disadvantage that it involves spinor components of opposite chirality. However, since we have reduced the space-time dimension, it is possible to apply a further redefinition,

$$
\psi^{i+}=\psi^{i}, \quad \psi^{i-}=-\varepsilon^{i j} \gamma_{4} \psi_{j},
$$

where the superscripts \pm denote the sign of the U(1) charge. Because of the presence of the matrix $\gamma_{4}$, the spinors are defined in the same eigenspace of $\gamma_{5}$ and we choose a positive eigenvalue, i.e.,

$$
\left(\gamma_{5}-\mathbf{1}\right) \psi^{i \pm}=0
$$

so that in the new basis we have replaced the doublets $\psi^{i}$ and $\psi_{i}$ of opposite chirality by four equal-chirality spinors $\psi^{i \pm}$. For the Dirac conjugate spinors, the corresponding relations follow from (B.3),

$$
\hat{\bar{\psi}}_{i+}=-\mathrm{i} \bar{\psi}_{i} \gamma_{4}, \quad \hat{\bar{\psi}}_{i-}=-\mathrm{i} \varepsilon_{i j} \bar{\psi}^{j},
$$

where on the left-hand side we have the $3 D$ Dirac conjugate spinors and on the right-hand side the $4 D$ conjugate spinors. Note that we have $\bar{\psi}_{i \pm}\left(\gamma_{5}-\mathbf{1}\right)=0$. In this basis the transformation rule (B.5) takes the form $(p, q=+,-)$,

$$
\psi^{i p} \rightarrow U^{i}{ }_{j} V^{p}{ }_{q} \psi^{j q},
$$


where $U$ denotes the chiral $\mathrm{SU}(2)$ transformation that was originally present in $4 D$, and $V$ the new $\mathrm{SU}(2)$ transformation that has emerged in $3 D$. In terms of the parameters in (B.5), we have

$$
V \approx \mathbf{1}+\frac{1}{2}\left(\begin{array}{cc}
\mathrm{i} \alpha & \beta \\
-\bar{\beta} & -\mathrm{i} \alpha
\end{array}\right) .
$$

The next topic is to derive the consequences of the Majorana property of the spinors. For chiral $4 D$ Majorana spinors the constraint on the chiral components is given by,

$$
\begin{aligned}
& C^{-1} \bar{\psi}_{i}^{\mathrm{T}}=\hat{C}^{-1} \hat{\bar{\psi}}_{i}^{\mathrm{T}}=\psi_{i}, \\
& C^{-1} \bar{\psi}^{i \mathrm{~T}}=\hat{C}^{-1} \hat{\bar{\psi}}^{i \mathrm{~T}}=\psi^{i},
\end{aligned}
$$

where the left-hand side contains the Dirac conjugate according to the $4 D$ and $3 D$ definition, respectively, where the indices are lowered or raised as a result of complex conjugation. From these constraints, one straightforwardly derives,

$$
\hat{C}^{-1} \hat{\bar{\psi}}_{i+}{ }^{\mathrm{T}}=\varepsilon_{i j} \gamma_{4} \psi^{j-}, \quad \hat{C}^{-1} \hat{\bar{\psi}}_{i-}{ }^{\mathrm{T}}=-\varepsilon_{i j} \gamma_{4} \psi^{j+} .
$$

Upon absorbing $\gamma_{4}$ into the definition of the charge conjugation matrix $\hat{C}$, one then proves the pseudo-reality relation

$$
C^{-1} \bar{\psi}_{i, p}^{\mathrm{T}}=\varepsilon_{i j} \varepsilon_{p q} \psi^{j, q} .
$$

Hence the appropriate charge conjugation matrix in the covariant $\mathrm{SU}(2) \times \mathrm{SU}(2)$ basis is given by,

$$
C=\hat{C} \gamma_{4},
$$

satisfying $C \gamma^{a} C^{-1}=-\gamma^{a \mathrm{~T}}$ with $C^{\mathrm{T}}=-C$. In (B.14) and (B.15) and henceforth we suppress the caret on $3 D$ quantities. The indices $p, q=+,-$ refer to the spinor components with positive and negative $\mathrm{U}(1)$ charge respectively. With these results we derive the Majorana re-ordering for fermionic bilinears,

$$
\bar{\psi}_{i, p} \Gamma \psi^{j, q}= \pm \varepsilon_{i k} \varepsilon^{j l} \varepsilon_{p r} \varepsilon^{q s} \bar{\psi}_{l, s} \Gamma \psi^{k, r},
$$

where the plus and the minus sign refer to $\Gamma=1$ and $\Gamma=\gamma^{\mu}$, respectively.

Finally we redefine the $4 D$ spinors such that the previous redefinitions can be applied uniformly. This is done by choosing a chiral Majorana spinor and modify it such that we obtain a field $\psi^{i}$ of positive chirality and positive $\mathrm{U}(1)$ charge. The field $\psi_{i}$ then follows from applying the $4 D$ Majorana condition. However, this only determines $\psi^{i}$ and $\psi_{i}$ up to a phase factor which implies that the $\mathrm{SU}(2)$ transformations induced by (B.5) on the underlying fields, are also determined up to phase factors. Insisting that the $3 D$ supersymmetry transformations are manifestly covariant with respect to the additional $\mathrm{SU}(2) \mathrm{R}$-symmetry component will fix these relative phase factors.

As an example let us start with the supersymmetry parameter $\epsilon_{i}$, which has positive $\mathrm{U}(1)$ charge and negative chirality. This identifies corresponding fields $\left(\psi^{i}, \psi_{i}\right)$ up to a phase factor $z$,

$$
\psi^{i}(\epsilon)=z \varepsilon^{i j} \gamma_{4} \epsilon_{j}, \quad \psi_{i}(\epsilon)=\bar{z} \varepsilon_{i j} \gamma_{4} \epsilon^{j}
$$


As long as we consider a single field, we are free to fix the phase factor, so we will eventually choose $z=1$. However, for the remaining spinors we should then leave the phase factor arbitrary. Hence for the remaining independent spinors we choose,

$$
\begin{aligned}
& \psi^{i}(\eta)=z_{\eta} \varepsilon^{i j} \eta_{j}, \quad \psi_{i}(\eta)=\bar{z}_{\eta} \varepsilon_{i j} \eta^{j}, \\
& \psi^{i}(\Omega)=z_{\Omega} \gamma_{4} \Omega^{i}, \quad \psi_{i}(\Omega)=\bar{z}_{\Omega} \gamma_{4} \Omega_{i}, \\
& \psi^{i}(\varphi)=z_{\varphi} \varphi^{i}, \quad \psi_{i}(\varphi)=\bar{z}_{\varphi} \varphi_{i} .
\end{aligned}
$$

The assignments of the conformal gauge fields $\psi_{\mu}{ }^{i}$ and $\phi_{\mu}{ }^{i}$ are the same as those of the transformation parameters $\epsilon^{i}$ and $\eta^{i}$, respectively. These ansätze now lead to the corresponding definitions of the quantities $\epsilon^{i p}, \eta^{i p}, \Omega^{i p}$ and $\varphi^{i p}$ which are all subject to the Majorana condition (B.14). They are summarized as follows,

$$
\begin{array}{rlrl}
\left.\epsilon^{i+}\right|_{3 D} & =\left.z \varepsilon^{i j} \gamma_{4} \epsilon_{j}\right|_{4 D}, & \left.\epsilon^{i-}\right|_{3 D} & =\left.\bar{z} \epsilon^{i}\right|_{4 D}, \\
\left.\eta^{i+}\right|_{3 D} & =\left.z_{\eta} \varepsilon^{i j} \eta_{j}\right|_{4 D}, & & \left.\eta^{i-}\right|_{3 D}=\left.\bar{z}_{\eta} \gamma_{4} \eta^{i}\right|_{4 D}, \\
\left.\Omega^{i+}\right|_{3 D}=\left.z_{\Omega} \gamma_{4} \Omega^{i}\right|_{4 D}, & & \left.\Omega^{i-}\right|_{3 D}=-\left.\bar{z}_{\Omega} \varepsilon^{i j} \Omega_{j}\right|_{4 D}, \\
\left.\varphi^{i+}\right|_{3 D}=\left.z_{\varphi} \varphi^{i}\right|_{4 D}, & & \left.\varphi^{i-}\right|_{3 D}=-\left.\bar{z}_{\varphi} \varepsilon^{i j} \gamma_{4} \varphi_{j}\right|_{4 D}, \\
\left.\chi^{i+}\right|_{3 D}=\left.z_{\chi} \varepsilon^{i j} \gamma_{4} \chi_{j}\right|_{4 D}, & & \left.\chi^{i-}\right|_{3 D}=\left.\bar{z} \chi \chi^{i}\right|_{4 D} .
\end{array}
$$

For the convenience of the reader we also add the expressions for the Dirac conjugate spinors,

$$
\begin{aligned}
\left.\bar{\epsilon}_{i+}\right|_{3 D} & =\left.\mathrm{i} \bar{z} \varepsilon_{i j} \bar{\epsilon}^{j}\right|_{4 D}, & & \left.\bar{\epsilon}_{i-}\right|_{3 D}=-\left.\mathrm{i} z \bar{\epsilon}_{i} \gamma_{4}\right|_{4 D}, \\
\left.\bar{\eta}_{i+}\right|_{3 D} & =-\left.\mathrm{i} \bar{z}_{\eta} \varepsilon_{i j} \bar{\eta}^{j} \gamma_{4}\right|_{4 D}, & & \left.\bar{\eta}_{i-}\right|_{3 D}=\left.\mathrm{i} z_{\eta} \bar{\eta}_{i}\right|_{4 D}, \\
\left.\bar{\Omega}_{i+}\right|_{3 D} & =\left.\mathrm{i} \bar{z}_{\Omega} \bar{\Omega}_{i}\right|_{4 D}, & & \left.\bar{\Omega}_{i-}\right|_{3 D}=\left.\mathrm{i} z_{\Omega} \varepsilon_{i j} \bar{\Omega}^{j} \gamma_{4}\right|_{4 D}, \\
\left.\bar{\varphi}_{i+}\right|_{3 D} & =-\left.\mathrm{i} \bar{z}_{\varphi} \bar{\varphi}_{i} \gamma_{4}\right|_{4 D}, & & \left.\bar{\varphi}_{i-}\right|_{3 D}=-\left.\mathrm{i} z_{\varphi} \varepsilon_{i j} \bar{\varphi}^{j}\right|_{4 D}, \\
\left.\bar{\chi}_{i+}\right|_{3 D} & =\left.\mathrm{i} \bar{z} \chi \varepsilon_{i j} \bar{\chi}^{j}\right|_{4 D}, & & \left.\bar{\chi}_{i-}\right|_{3 D}=-\left.\mathrm{i} z_{\chi} \bar{\epsilon}_{i} \gamma_{4}\right|_{4 D} .
\end{aligned}
$$

In the main text we have defined the set of phase factors consistent with supersymmetry and R-symmetry, as

$$
z=1, \quad z_{\eta}=-\mathrm{i}, \quad z_{\Omega}=1, \quad z_{\phi}=\mathrm{i} \quad z_{\chi}=1 .
$$

Open Access. This article is distributed under the terms of the Creative Commons Attribution License (CC-BY 4.0), which permits any use, distribution and reproduction in any medium, provided the original author(s) and source are credited.

\section{References}

[1] S. Cecotti, S. Ferrara and L. Girardello, Geometry of Type II Superstrings and the Moduli of Superconformal Field Theories, Int. J. Mod. Phys. A 4 (1989) 2475 [inSPIRE].

[2] S. Ferrara and S. Sabharwal, Quaternionic Manifolds for Type II Superstring Vacua of Calabi-Yau Spaces, Nucl. Phys. B 332 (1990) 317 [inSPIRE].

[3] B. de Wit, F. Vanderseypen and A. Van Proeyen, Symmetry structure of special geometries, Nucl. Phys. B 400 (1993) 463 [hep-th/9210068] [INSPIRE].

[4] J. De Jaegher, B. de Wit, B. Kleijn and S. Vandoren, Special geometry in hypermultiplets, Nucl. Phys. B 514 (1998) 553 [hep-th/9707262] [INSPIRE]. 
[5] B. de Wit and F. Saueressig, Off-shell $N=2$ tensor supermultiplets, JHEP 09 (2006) 062 [hep-th/0606148] [INSPIRE].

[6] M. Dine, P.Y. Huet and N. Seiberg, Large and Small Radius in String Theory, Nucl. Phys. B 322 (1989) 301 [inSPIRE].

[7] J. Dai, R.G. Leigh and J. Polchinski, New Connections Between String Theories, Mod. Phys. Lett. A 4 (1989) 2073 [inSPIRE].

[8] M. Abou-Zeid, B. de Wit, D. Lüst and H. Nicolai, Space-time supersymmetry, IIA/B duality and M-theory, Phys. Lett. B 466 (1999) 144 [hep-th/9908169] [INSPIRE].

[9] P. Candelas, X.C. De la Ossa, P.S. Green and L. Parkes, An Exactly soluble superconformal theory from a mirror pair of Calabi-Yau manifolds, Phys. Lett. B 258 (1991) 118 [INSPIRE].

[10] N. Banerjee, B. de Wit and S. Katmadas, The Off-Shell 4D/5D Connection, JHEP 03 (2012) 061 [arXiv:1112.5371] [INSPIRE].

[11] B. de Wit, M. Roček and S. Vandoren, Hypermultiplets, hyperKähler cones and quaternion Kähler geometry, JHEP 02 (2001) 039 [hep-th/0101161] [INSPIRE].

[12] M. Roček, C. Vafa and S. Vandoren, Hypermultiplets and topological strings, JHEP 02 (2006) 062 [hep-th/0512206] [INSPIRE].

[13] M. Roček, C. Vafa and S. Vandoren, Quaternion-Kähler spaces, hyperKähler cones and the c-map, math/0603048 [INSPIRE].

[14] I. Antoniadis, E. Gava, K.S. Narain and T.R. Taylor, Topological amplitudes in string theory, Nucl. Phys. B 413 (1994) 162 [hep-th/9307158] [InSPIRE].

[15] P.S. Howe, J.M. Izquierdo, G. Papadopoulos and P.K. Townsend, New supergravities with central charges and Killing spinors in (2+1)-dimensions, Nucl. Phys. B 467 (1996) 183 [hep-th/9505032] [INSPIRE].

[16] E.A. Bergshoeff, O. Hohm, J. Rosseel and P.K. Townsend, On Maximal Massive 3D Supergravity, Class. Quant. Grav. 27 (2010) 235012 [arXiv:1007.4075] [INSPIRE].

[17] S.M. Kuzenko, U. Lindström and G. Tartaglino-Mazzucchelli, Off-shell supergravity-matter couplings in three dimensions, JHEP 03 (2011) 120 [arXiv:1101.4013] [INSPIRE].

[18] U. Gran, J. Greitz, P.S. Howe and B.E.W. Nilsson, Topologically gauged superconformal Chern-Simons matter theories, JHEP 12 (2012) 046 [arXiv:1204.2521] [INSPIRE].

[19] D. Butter, S.M. Kuzenko, J. Novak and G. Tartaglino-Mazzucchelli, Conformal supergravity in three dimensions: New off-shell formulation, JHEP 09 (2013) 072 [arXiv:1305.3132] [INSPIRE].

[20] D. Butter, S.M. Kuzenko, J. Novak and G. Tartaglino-Mazzucchelli, Conformal supergravity in three dimensions: Off-shell actions, JHEP 10 (2013) 073 [arXiv:1306.1205] [INSPIRE].

[21] N. Berkovits and W. Siegel, Superspace effective actions for 4-D compactifications of heterotic and type-II superstrings, Nucl. Phys. B 462 (1996) 213 [hep-th/9510106] [INSPIRE].

[22] B. de Wit, S. Katmadas and M. van Zalk, New supersymmetric higher-derivative couplings: Full $N=2$ superspace does not count!, JHEP 01 (2011) 007 [arXiv: 1010.2150] [inSPIRE].

[23] M. Roček and P. van Nieuwenhuizen, $N \geq 2$ supersymmetric Chern-Simons terms as $D=3$ extended conformal supergravity, Class. Quant. Grav. 3 (1986) 43 [INSPIRE]. 
[24] U. Lindström and M. Roček, Superconformal Gravity in Three-dimensions as a Gauge Theory, Phys. Rev. Lett. 62 (1989) 2905 [InSPIRE].

[25] B. de Wit, J.W. van Holten and A. Van Proeyen, Structure of $N=2$ Supergravity, Nucl. Phys. B 184 (1981) 77 [Erratum ibid. B 222 (1983) 516] [INSPIRE].

[26] B. de Wit, B. Kleijn and S. Vandoren, Superconformal hypermultiplets, Nucl. Phys. B 568 (2000) 475 [hep-th/9909228] [inSPIRE].

[27] U. Lindström and M. Roček, Scalar Tensor Duality and $N=1, N=2$ Nonlinear $\sigma$-models, Nucl. Phys. B 222 (1983) 285 [INSPIRE].

[28] E. Bergshoeff, M. de Roo and B. de Wit, Extended Conformal Supergravity, Nucl. Phys. B 182 (1981) 173 [inSPIRE].

[29] B. de Wit, R. Philippe and A. Van Proeyen, The Improved Tensor Multiplet in $N=2$ Supergravity, Nucl. Phys. B 219 (1983) 143 [INSPIRE].

[30] D. Butter, B. de Wit, S.M. Kuzenko and I. Lodato, New higher-derivative invariants in $N=2$ supergravity and the Gauss-Bonnet term, JHEP 12 (2013) 062 [arXiv:1307.6546] [INSPIRE].

[31] S. Katmadas and R. Minasian, $\mathcal{N}=2$ higher-derivative couplings from strings, JHEP 02 (2014) 093 [arXiv: 1311.4797] [INSPIRE]. 